\title{
Advanced Monte Carlo dose calculations for eye plaque brachytherapy
}

by

\section{Zack Parsons}

A thesis submitted to the Faculty of Graduate and Postdoctoral Affairs in partial fulfillment of the requirements for the degree of

Master of Science

in

Physics

Specialization in Medical Physics

Ottawa-Carleton Institute for Physics

Department of Physics

Carleton University

Ottawa, Ontario, Canada

2018

(C) 2018 Zack Parsons 



\section{Abstract}

Eye plaque brachytherapy is a radiation therapy technique designed for treating ocular cancers. Current clinical radiation dose evaluations for eye plaque brachytherapy involve considerable assumptions, resulting in inaccurate doses. This thesis aims to advance dose evaluations for eye plaque brachytherapy by implementation of detailed models for various photon and beta plaques in the egs_brachy Monte Carlo code. Calculated dose distributions are verified against published results. These plaque models and dose distributions will be distribued to the medical physics community. Dose distributions are calculated for differenct scenarios (clinical assumptions; plaque in water; plaque in realistic eye model). These results confirm the importance of model-based calculations for photon plaques and show that modelling tissues should be considered for beta plaques. Finally, dose distributions for photon and beta plaques are compared, demonstrating the potential of model-based dose evaluations in future studies. 


\section{Acknowledgments}

Without the support of my girlfriend, Hayley, my parents Chris and Cheryl, and my siblings Dustin, James, and Jesse, this thesis would never have taken shape. Thank you for always being there.

I would like to thank my supervisor Dr. Rowan Thomson for her infinite patience and her advice in navigating the often bewildering currents of graduate research.

I thank Stephen Deering and Marc Chamberland for laying the groundwork for the creation and use of eye plaque models in egs_brachy.

Finally, my colleagues and friends among the Carleton Laboratory for Radiotherapy Physics deserve thanks: Martin Martinov, Stephen Deering, Patty Oliver, Luke McCooeye, Viktor Malkov, and Suxer Gracia. They all helped in any way they could, and for that I am eternally grateful. 


\section{Statement of originality}

This thesis contains the large majority of work which was undertaken during the author's Master's degree at Carleton University. Many of the results in the following pages form the basis for a future database of geometry and dose distribution files and an accompanying paper. All calculations using egs_brachy in this work were done by the author.

Dr. Rowan Thomson supervised the author and contributed to every step of this work. She was the source of the initial project idea, and offered guidance throughout the work, including the final thesis revisions.

Stephen Deering and Dr. Marc Chamberland created initial eye plaque geometries for use with egs_brachy. Their designs were used as a point of comparison and a source for ideas in the design of the plaque geometries presented here. The model of the human eye used for calculations throughout this work was developed by Marielle Lesperance. 


\section{Contents}

Abstract $\quad$ ii

Acknowledgments $\quad$ iii

Statement of originality iv

Table of contents vii

List of tables viii

List of figures $\quad \mathrm{x}$

Nomenclature and notation $\quad$ xi

1 Introduction 1

1.1 Ocular cancer ........................ . . . 1

1.2 Radiation therapy and dose . . . . . . . . . . . . . . . . . 2

1.3 Eye plaque brachytherapy . . . . . . . . . . . . . . . . . . 2

1.4 Treatment planning . . . . . . . . . . . . . . . . 4

1.4.1 Motivation for advanced dose calculations . . . . . . . . . 7

1.5 Model-based and Monte Carlo dose calculations . . . . . . . . . . . . 13

1.6 Thesis purpose . . . . . . . . . . . . . . . . . . 14

1.7 Thesis outline . . . . . . . . . . . . . . . 16 
2 Methods $\quad 17$

2.1 Eye plaque model geometries _. . . . . . . . . . . . . . . . 18

2.1.1 Photon plaque geometries . . . . . . . . . . . . . 20

2.1.2 Beta plaque geometries . . . . . . . . . . . . . . 27

2.2 Phantom geometry . . . . . . . . . . . . . . . . . 31

2.2.1 Voxelized eye phantom model . . . . . . . . . . . . . . 32

2.3 Dose calculations . . . . . . . . . . . . . . . . . . . . . . . 35

2.3.1 Simulation parameters . . . . . . . . . . 36

2.3.2 Dose normalization . . . . . . . . . . . . . 37

2.4 Calculation specifications . . . . . . . . . . . . . 38

2.4.1 Uncertainties ................... . . 40

$\begin{array}{lll}3 & \text { Results } & 42\end{array}$

3.1 Photon plaque results . . . . . . . . . . . . . . . 42

3.1.1 Comparison of $D_{T G 43}, D_{w, w}$, and $D_{m, m} \ldots \ldots . . . . . \quad 54$

3.2 Beta plaque results f . . . . . . . . . . . . . . . . 58

3.2.1 Comparison of CIA beta plaque models . . . . . . . . . . . . 64

3.3 Photon and beta plaque comparisons . . . . . . . . . . . . . 66

$\begin{array}{lll} & \text { Discussion } & 71\end{array}$

4.1 Verification of egs_brachy and eye plaque models . . . . . . . . . 71

4.1.1 Photon plaque verification ............. . . 71

4.1.2 Beta plaque verification .............. 73

4.2 Comparison of $D_{T G 43}, D_{w, w}$, and $D_{m, m} \ldots \ldots \ldots$. . . . . . 75

4.3 Comparison of photon and beta plaque dose distributions . . . . . . 79

5 Conclusions and outlook $\quad 81$ 
Appendix

A Photon plaque seed positions

References 


\section{List of Tables}

2.1 Photon plaque dimensions . . . . . . . . . . . . . . . . 25

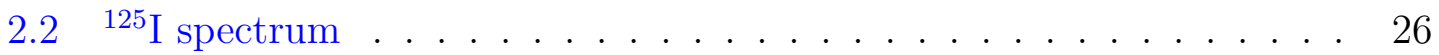

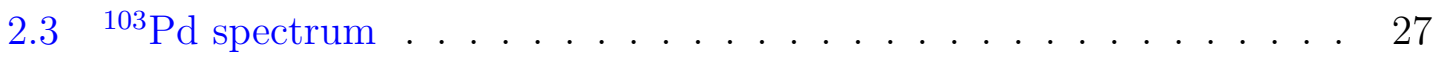

2.4 Beta plaque dimensions . . . . . . . . . . . . . . . . . . . . . . . . . $\quad 29$

2.5 Scoring region dimensions $\ldots \ldots \ldots$

2.6 Eye phantom composition . . . . . . . . . . . . . . . . . . 34

2.7 Photon and beta transport parameters $\ldots \ldots \ldots \ldots$

2.8 Source parameters . . . . . . . . . . . . . . . . . . . . . . . 38

$3.1 D_{T G 43}$ and ratios at points of interest $\ldots \ldots \ldots \ldots \ldots$

$3.2 D_{w, w}$ and ratios at points of interest $\ldots \ldots \ldots \ldots \ldots \ldots$

3.3 Eye structure dose comparison between $D_{T G 43}, D_{w, w}$, and $D_{m, m} \ldots 57$

3.4 Points of interest comparison between COMS $16 \mathrm{~mm}$ and CCB . . . 69

3.5 Eye structure dose comparison between COMS $16 \mathrm{~mm}$ and CCB . . 70

A.1 Seed position coordinates $\ldots \ldots \ldots \ldots \ldots \ldots$ 


\section{List of Figures}

1.1 TG43 geometry . . . . . . . . . . . . . . . . 6

1.2 Photon mass-energy absorption coefficents of eye tissues . . . . . . 11

1.3 Electron stopping powers of eye tissues . . . . . . . . . . . . . 12

2.1 Eye coordinate systems . . . . . . . . . . . . . . . . . . . 18

2.2 Plaque models in model of eye . . . . . . . . . . . . . . . . 19

2.3 COMS seed positions . . . . . . . . . . . . . . 21

2.4 COMS eye plaque geometric parameters $\ldots \ldots \ldots \ldots \ldots$

2.5 COMS plaque regions $\ldots \ldots \ldots \ldots \ldots \ldots \ldots$

2.6 Photon brachytherapy seed geometries $\ldots \ldots \ldots \ldots$

2.7 Beta plaque geometry definition . . . . . . . . . . . . . 29

2.8 CIA plaque geometry model $\ldots \ldots \ldots \ldots \ldots$

$3.1 D_{T G 43}$ central axis depth-dose curves: ${ }^{125} \mathrm{I} \ldots \ldots \ldots \ldots \ldots$

$3.2 \quad D_{T G 43}$ central axis depth-dose curves: ${ }^{103} \mathrm{Pd} \ldots \ldots \ldots$

$3.3 D_{w, w}$ central axis depth-dose curves: ${ }^{125} \mathrm{I} \ldots \ldots \ldots \ldots$

$3.4 D_{w, w}$ central axis depth-dose curves: ${ }^{103} \mathrm{Pd} \ldots \ldots \ldots \ldots \ldots$ 
3.5 A three-dimensional $D_{w, w}$ comparison of egs_brachy and BrachyDose 53

3.6 Photon plaque isodose contours . . . . . . . . . . . . . . 55

3.7 Photon plaque $D_{w, w} / D_{T G 43}$ and $D_{m, m} / D_{T G 43}$ ratios $\ldots \ldots .56$

3.8 Beta plaque central axis relative depth-dose curves $\ldots \ldots \ldots$. . . . 60

3.9 CCB central axis dose per decay with depth $\ldots \ldots \ldots \ldots$. . . . . 61

3.10 Beta plaque lateral axis dose profiles: Planar, CCA, CCB, and CCC . 62

3.11 Beta plaque lateral axis dose profiles: CCD, CGD, CCX, and CXS . 63

3.12 CIA plaque central axis and notched axis doses $\ldots \ldots \ldots \ldots$

3.13 CIA plaque isodose contours . . . . . . . . . . . 65

3.14 Depth-dose comparisons between COMS $16 \mathrm{~mm}$ and CCB beta plaque 67

3.15 isodose contour comparison between COMS $16 \mathrm{~mm}$ and CCB beta

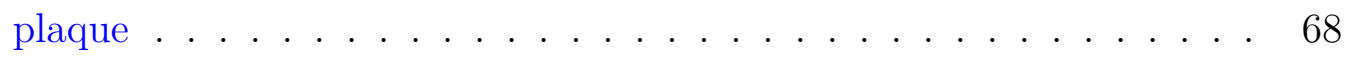




\title{
Nomenclature and notation
}

\author{
AAPM ....... American Association of Physicists in Medicine \\ CLRP ........ Carleton Laboratory for Radiotherapy Physics \\ COMS ........ Collaborative Ocular Melanoma Study \\ $\dot{D}(r, \theta) \ldots \ldots \ldots$. Dose rate \\ $D_{m, m} \ldots \ldots \ldots . .$. Dose with plaque in voxelized, tissue accurate eye phantom \\ $D_{T G 43} \ldots \ldots \ldots .$. Dose from superposition of seeds in water \\ $D_{w, w} \ldots \ldots \ldots \ldots . .$. Dose with plaque in voxelized water phantom \\ $F(r, \theta) \ldots \ldots \ldots .2 \mathrm{D}$ anisotropy function \\ $g_{L}(r) \ldots \ldots \ldots \ldots$ TG43 radial dose function \\ $G_{L}(r, \theta) \ldots \ldots$. TG43 geometry function \\ Gy .............. Gray [joule / kilogram] \\ $K_{\text {col }}$............. Collision kerma \\ MBDCA ..... Model based dose calculation algorithm \\ MC .............. Monte Carlo \\ $R_{C S D A} \ldots \ldots \ldots$ Continuous slowing down approximation range \\ $S_{K} \ldots \ldots \ldots \ldots \ldots$ Air-kerma strength \\ $S_{K}^{\text {hist }} \ldots \ldots \ldots \ldots$....... Air-kerma strength per history \\ $S / \rho \ldots \ldots \ldots \ldots \ldots$........ Stopping power \\ TG43 .......... AAPM Task Group No. 43 \\ TG129 ........ AAPM Task Group No. 129 \\ TG186 ........ AAPM Task Group No. 186 \\ $Z_{\text {eff } \ldots \ldots \ldots \ldots \ldots . . . . . . .}$ Effective atomic number \\ $\Lambda \ldots \ldots \ldots \ldots \ldots . . . .$. Dose rate constant
}


$\mu_{e n} / \rho \ldots \ldots \ldots . . .$. Mass-energy absorption coefficient

$\rho \ldots \ldots \ldots \ldots \ldots . . . . . .$. Mass density $\left[\mathrm{g} / \mathrm{cm}^{3}\right]$

$\phi_{a n}(r) \ldots \ldots \ldots . .1 \mathrm{D}$ anisotropy function

$\Phi_{E} \ldots \ldots \ldots \ldots \ldots$....... Photon fluence differential in energy

$(x, y, z) \ldots \ldots .$. Eye plaque coordinate system

$(\mathrm{X}, \mathrm{Y}, \mathrm{Z}) \ldots$. Eye centre coordinate system 


\section{Chapter 1}

\section{Introduction}

\subsection{Ocular cancer}

There are several forms that cancer can take within the eye including ocular melanoma, intraocular lymphoma and retinoblastoma. There are 2500 reported cases of uveal melanoma per year in the United States, making it the most common form of ocular cancer ${ }^{1}$. Uveal melanoma arises from the uveal layer of the eye, which is the tissue layer that contains the iris, the choroid, and the cilary body. Uveal melanoma is usually diagnosed through a clinical examination with biomicroscopy and ophthalmoscopy and can be further confirmed using ultrasonography, fundus fluorescein angiography, or optical coherence tomography ${ }^{2}$. Previously, the most common treatment for ocular melanoma was enucleation, which is a full removal of the eye. While enucleation offers a high level of local tumor control, loss of the eye is not a desired outcome. In the pursuit of treatments which allow for vision retention, radiation therapy offers numerous choices. Radiation therapy techniques used for ocular cancer treaments include external beam therapy of various types such as: photon ${ }^{3,4}$, $\operatorname{proton}^{5,6,7,8}$, helium-ion ${ }^{9}$, and Gamma Knife ${ }^{10,11,12}$. Eye plaque brachytherapy is a technique that was specifically developed for ocular cancer treatment and is the focus of this thesis. 


\subsection{Radiation therapy and dose}

Radiation therapy is a cancer treatment that uses ionizing radiation to deliver 'dose' to a cancerous region in an attempt to damage the cancerous cells ${ }^{13}$. Dose is an important quanitity in radiation physics that gives the energy deposited in a medium by ionizing radiation per unit mass. The unit for dose is the Gray (Gy), which is

given by joules per kilogram in SI units $\left[\mathrm{Gy}=\frac{\mathrm{J}}{\mathrm{kg}}\right]$. If enough dose is delivered to the cancerous cells then they will experience cell death via different mechanisms such as apoptosis (a form of programmed cell death), mitotic catastrophe (the primary form of cell deaths from ionizing radiation, caused by abnormal entry to mitosis), and senescence (a state where damaged cells no longer replicate) ${ }^{14,15}$. Unfortunately, by the same mechanisms radiation therapy can damage and kill healthy tissue. When performing radiation therapy treatments care is taken to prevent excess damage to healthy tissues; this is where treatment planning and dose evaluation come in. During treatment evaluation, dose calculations are made to determine an appropriate radiation treatment for the patient, where an appropriate treatment attempts to deposit the majority of energy in the cancerous tissue and limits the energy deposited to healthy tissues. For treatment evaluation to be as effective as possible, advancing the accuracy and availability of dose evaluation methods used for treatment evaluation is crucial.

\subsection{Eye plaque brachytherapy}

Brachytherapy is a radiotherapy (another term for radiation therapy) cancer treatment that involves radioactive sources which are placed near or in the area to be treated $^{16}$. Brachytherapy sources traditionally come in the form of 'seeds'. These seeds are small (a few $\mathrm{mm}$ in length) and contain a radioisotope such as ${ }^{125} \mathrm{I},{ }^{103} \mathrm{Pd}$ 
or ${ }^{131} \mathrm{Cs}$. The photons emitted by brachytherapy seeds tend to have a much lower energy than external beam treatments. For example, ${ }^{125} \mathrm{I}$ has a mean photon energy of $28 \mathrm{keV}$ and ${ }^{103} \mathrm{Pd}$ a photon energy of $21 \mathrm{keV}$ compared to typical external beam energies that are over $1 \mathrm{MeV}$. The lower energy of these treatments allows for a steep drop off in photon fluence and gives the ability to deposit energy local to the treatment target while avoiding excessive dose to organs at risk further from the seeds.

Eye plaque brachytherapy is a specific type of brachytherapy used to treat ocular cancers. Eye plaque brachytherapy was developed with the purpose of retaining vision while treating cancer. The first recorded attempt at using brachytherapy for eye treatments was using radon seeds in $1930^{[17]}$. Since then many different sources have been used including ${ }^{60} \mathrm{Co}^{[18]},{ }^{106} \mathrm{Ru} /{ }^{106} \mathrm{Rh}^{[19,20,21]},{ }^{125} \mathrm{I}^{[22,23,24,25]},{ }^{103} \mathrm{Pd}^{[26,24,25,27]}$, ${ }^{198} \mathrm{Au}^{[28]},{ }^{90} \mathrm{Sr} /{ }^{90} \mathrm{Y}^{[21]}$, and ${ }^{131} \mathrm{Cs}^{[24,25]}$. Eye plaques are typically metallic plaques that come in various sizes and configurations. The plaques contain a radionuclide source in the form of multiple brachytherapy seeds or a film coated in a radioactive substance. The eye plaques are sutured onto the eye over the cancerous region and left in place for the duration of treatment time (which is generally on the order of 100 hours). The most common sources in use today $\left({ }^{125} \mathrm{I},{ }^{103} \mathrm{Pd},{ }^{131} \mathrm{Cs},{ }^{106} \mathrm{Ru} /{ }^{106} \mathrm{Rh}\right)$ are lower energy photon sources or beta sources.

The plaques themselves come in various designs, but a standard design was introduced during a large scale study that began in 1985 known as the Collaborative Ocular Melanoma Study (COMS) ${ }^{29}$. COMS was a multi-institutional clinical trial that used standarized plaque designs and treatment methods to examine the effectiveness of eye plaque brachytherapy treatments. The COMS study concluded that eye plaque brachytherapy is comparable to enucleation for tumor control ${ }^{30}$. A recent study concluded that eye plaque brachytherapy is more effective than proton therapy 
for tumor control ${ }^{31}$.

The COMS plaque designs are not the only plaque designs in use. Various other plaque models are used in clinics around the world. There are also silver plaque designs that utilize beta radiation as the active method of energy deposition. These plaques are more commonly used in Europe. The most popular beta plaque variants are ${ }^{106} \mathrm{Ru} /{ }^{106} \mathrm{Rh}$ designs created by Eckert \& Ziegler BEBIG of Berlin, Germany. The mean energy of ${ }^{106} \mathrm{Ru} /{ }^{106} \mathrm{Rh}$ beta particle emissions is $1.41 \mathrm{MeV}$ and the maximum energy is $3.54 \mathrm{MeV}$ which result in approximate ranges of about $7 \mathrm{~mm}$ and $15 \mathrm{~mm}$ in soft tissue respectively based on the assumption of continuous energy loss as the electrons traverse the medium (this assumed range is known as $R_{C S D A}$, where CSDA stands for continuous slowing down approximation). The short range of electrons in tissue allows for a high deposition of energy in the tumor region and limited deposition of energy in surrounding tissues and organs at risk ${ }^{32}$.

\subsection{Treatment planning}

Today, most dose calculations for treatment evaluation of photon eye plaque brachytherapy are done using a protocol created by the American Association of Physicists in Medicine (AAPM) Task Group 43 (TG43) ${ }^{33,34}$. The TG43 protocol includes various assumptions that make the calculation of dose easier. Among these assumptions is that the patient is made purely of water, the presence of the eye plaque is ignored, and any interseed effects (interaction of photons emitted from a seed with other nearby seeds) that may occur are also ignored. When using the TG43 protocol eye plaque dose calculations are often done such that a dose of 85 Gy is prescribed to the tumor apex ${ }^{35}$.

The TG43 formalism gives two possible equations, one for one dimensional (1D) 
(referring to the fact that the source is in effect treated as a 'point' source) calculations and one for two dimensional (2D) (referring to the fact that the source is in effect treated as a 'line' source) calculations. The generalized 1D brachytherapy dose rate formula given by the TG43 protocol is,

$$
\dot{D}(r, \theta)=S_{K} \Lambda \frac{G_{L}(r, \theta)}{G_{L}\left(r_{0}, \theta_{0}\right)} g_{L}(r) \phi_{a n}(r)
$$

and for the $2 \mathrm{D}$ case,

$$
\dot{D}(r, \theta)=S_{K} \Lambda \frac{G_{L}(r, \theta)}{G_{L}\left(r_{0}, \theta_{0}\right)} g_{L}(r) F(r, \theta)
$$

where $r$ is the distance to the point of interest from the centre of the source; $r_{0}$ is the given protocol reference distance of $1 \mathrm{~cm} ; \theta$ is the polar angle at the point of interest with respect to the longitudinal axis of the source; $\theta_{0}$ is a protocol reference of $90^{\circ}$ and defines the transverse plane of the source; $S_{K}$ is the air-kerma strength given by $S_{K}=K_{\delta}(d) d^{2}$ (the air-kerma rate in vacuo at distance $d$ multiplied by that distance squared); $\Lambda$ is the dose-rate constant in water, $\frac{\dot{D}\left(r_{0}, \theta_{0}\right)}{S_{K}} ; G_{L}(r, \theta)$ is the geometry function that gives an inverse square-law correction to the dose rate; $g_{L}(r)=\frac{\dot{D}\left(r, \theta_{0}\right)}{\dot{D}\left(r_{0}, \theta_{0}\right)} \frac{G_{L}\left(r_{0}, \theta_{0}\right)}{G_{L}\left(r, \theta_{0}\right)}$ is the radial dose function that accounts for the effects of photon attenuation and scattering on the dose rate with respect to the radius r; and $F(r, \theta)$, called the 2D anisotropy function, where $F(r, \theta)=\frac{\dot{D}(r, \theta)}{\dot{D}\left(r, \theta_{0}\right)} \frac{G_{L}\left(r, \theta_{0}\right)}{G_{L}(r, \theta)}$ accounts for the angular dependent attenuation and scattering effects on the dose rate. In the 1D case $F(r, \theta)$ is replaced by $\phi_{a n}(r)$, the $1 \mathrm{D}$ anisotropy function which is $\frac{\int_{0}^{\pi} \dot{D}(r, \theta) \sin (\theta) d \theta}{2 \dot{D}\left(r, \theta_{0}\right)}$ or the ratio of solid angle weight dose rate to the on the traverse plane at the same distance. For reference, $r$ and $\theta$ are defined in Figure 1.1.

However, TG43 is a dose calculation protocol specific to photon brachytherapy 


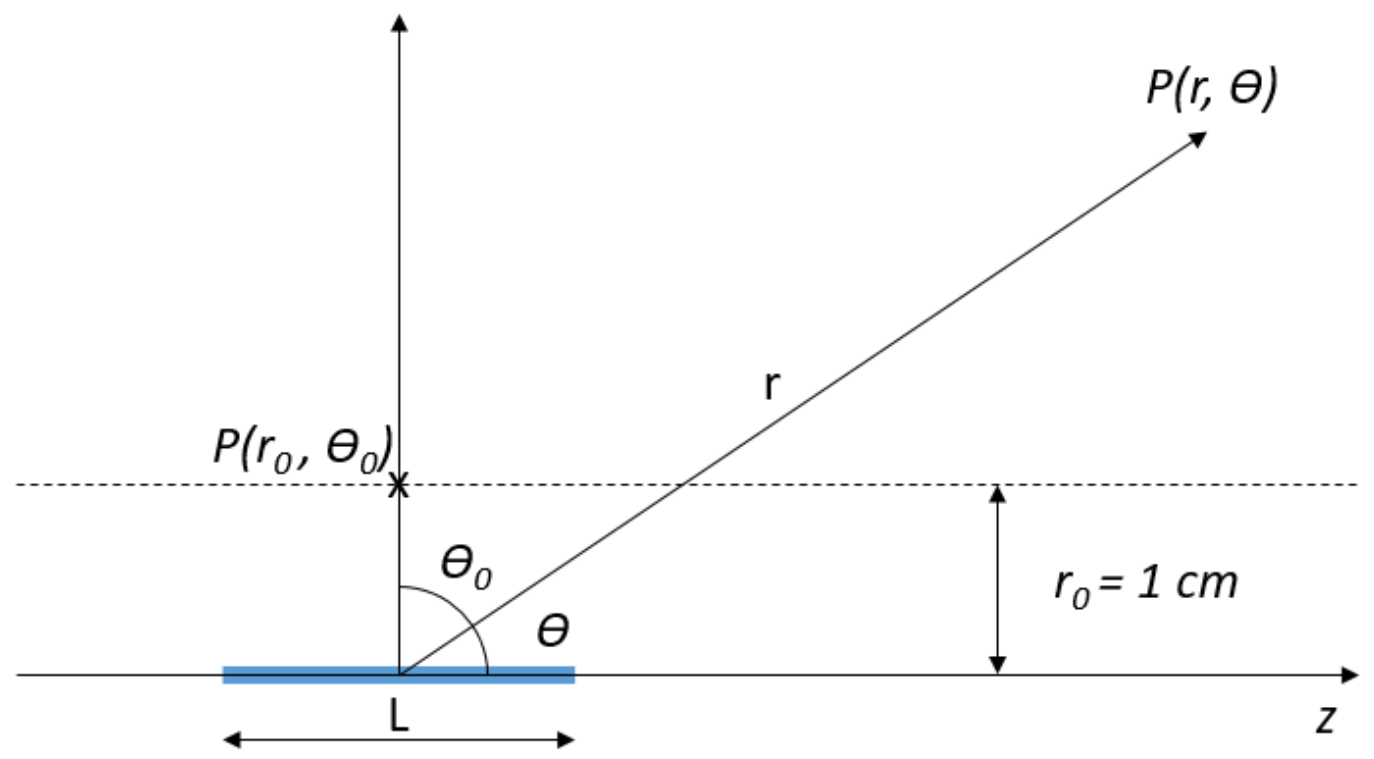

Figure 1.1: Schematic diagram of the geometry and variables used for the TG43 formalism. 
and is not appropriate for beta plaque dose calculations. Beta plaque treatment planning and dose evaluation do not have a standardized protocol to follow and various methods may be used when planning a treatment. The calculation of dose for beta plaques is often done using 1D methods along the axis defined from the centre of the plaque and therefore only considers depth and cannot account for organs at risk outside of the sclera. These 1D calculations are done with knowledge of the plaque source activity to calculate time needed to reach the prescription dose at tumor apex. $2 \mathrm{D}$ or $3 \mathrm{D}$ calculations in the clinic are limited to hand calculations or Plaque Simulator, a commercially available but non-FDA approved eye plaque treatment planning system created by Eye Physics, LLC of Los Alamitos, CA (which may also be used for photon eye plaque dose calculations).

\subsubsection{Motivation for advanced dose calculations}

The assumptions employed by the TG43 approach create discrepancies between calculated and delivered doses, this can be seen both from a physics stand point and from the conclusions of recent works on the subject of model-based dose calculations. Various reports and studies recommend including a model-based approach in treatment planning and evaluation including the AAPM reports TG129 ${ }^{[36]}$ and TG186 ${ }^{[37]}$.

To understand why the assumptions of TG43 break down an understanding of photon interactions with matter is needed. Photon interactions with matter come in one of four types: the photoelectric effect, Compton scattering, Rayleigh scattering, and pair/triplet production. Photon interactions with matter result in an attenuation of incoming photons which can lower photon fluence (number of photons crossing a perpendicular area) and energy fluence (amount of energy crossing a perpendicular 
area) as the photons traverse a given medium. At low energies photon interactions with matter are dominated by the photoelectric effect. Compton scattering is the second most important type of photon interaction at low energy levels and is the result of a photon interacting with a charged particle (such as an electron). Compton scattering is inelastic and results in a loss of energy and a change of direction for the photon and a gain of some recoil energy by the electron. Pair/triplet production is an interaction where a photon of high enough energy causes the creation of an electron-positron pair. Pair/triple production does not occur at the low energies of photon brachytherapy seeds. Rayleigh scattering is only a minor contributor to photon interactions with matter. Rayleigh scattering is elastic and the incident photons do not lose energy (and so do not deposit any energy in a medium), though their direction changes. Compton scattering is only linearly dependent on $Z_{\text {eff }}$ (the effective atomic number of a medium) and does not see a great increase in occurrence with change in $Z_{\text {eff }}$; however, the dominating photoelectric effect is highly $Z_{\text {eff }}$ dependent (its cross-section per atom is related to $\frac{Z^{n}}{h \nu^{3}}$ where $n$ can vary between 4 and 5) ${ }^{38}$. The high $Z_{\text {eff }}$ dependence of the photoelectric effect establishes that at low energies the probability of a photon interaction in matter occurring becomes highly dependent on material composition and small change in composition could greatly affect the final dose. The increased dependence on material composition is especially relevant for eye plaques where the plaques themselves act as shielding and are made out of much higher $Z_{\text {eff }}$ components than the tissue media being irradiated.

To understand where the probabilities of photon interactions in matter and their dependence on $Z_{\text {eff }}$ come into play it is important to note how absorbed dose is calculated for low energy photons. Typically when considering low energy photon sources like brachytherapy seeds, absorbed dose is assumed to be equal to the collision KERMA (Kinetic Energy Released per unit MAss). Kerma is the quantity used to 
discuss energy transferred to charged particles in a medium by non-ionizing particles such as photons. Kerma has both a radiative kerma component (energy transferred that results in loss to radiative interactions) and a collision kerma component (energy transferred that results in local energy deposition through electron ionization and excitation). The assumption of equivalency between absorbed dose and collision kerma is viable because the range of electrons at such low energies is very short in comparison to the considered scale. At low energies absorbed dose for a spectrum of photons is given by the general definition of collision kerma,

$$
D \simeq K_{c o l}=\int_{0}^{E_{\max }} E \Phi_{E}\left(\frac{\mu_{e n}(E)}{\rho}\right)_{m} d E
$$

where $\Phi_{E}$ is the photon fluence differential in energy $\left(\frac{d \Phi}{d E}\right)$, and $\left(\frac{\mu_{e n}(E)}{\rho}\right)_{m}$ is the mass-energy absorption coefficient for the photon energy $E$ in medium $m$. These quantities are all integrated up to the maximum energy of the incident photon spectrum. Photons deposit energy in a medium through the photon interactions discussed previously, and because of this the mass-energy absorption coefficient, $\left(\frac{\mu_{e n}}{\rho}\right)$ depends on the probabilities of photon interactions which in turn depend on $Z_{\text {eff }}$ at low energies.

The TG43 formalism treats the patient as an infinite body of water, where as real human tissue and organ compositions can differ markedly from water and each other. To see the effect change in medium can have on $\left(\frac{\mu_{e n}}{\rho}\right)_{m}$ refer to Figure 1.2 which shows the ratios of $\left(\frac{\mu_{e n}}{\rho}\right)_{m}$ for different eye tissues to $\left(\frac{\mu_{e n}}{\rho}\right)_{\text {water }}$. TG43 also does not take into account the shielding effects of the eye plaque or photon attenuation caused by the high $\mathrm{Z}$ materials that seeds are often made of (e.g. titanium and lead are components of the ${ }^{103} \mathrm{Pd}$ Theragenics TheraSeed 200, titanium and silver are components of the ${ }^{125}$ I OncoSeed 6711, etc.). The limitations of the TG43 approach 
motivate the development and application of advanced dose calculation algorithms.

Charged particles (such as electrons) do not interact with matter in the same way that nonionizing particles (such as photons) do. An incident electron may interact with matter in one of three ways: an elastic collision with the matter, an inelastic collision with bound electrons, or a radiative interaction with the nucleus. Elastic collisions result in no energy loss for the incident electron and so do not deposit energy in the medium. Inelastic collisions with a bound electron result in a transfer of energy to that electron which can result in excitation or ionization. Radiative interactions with the nucleus result in the deceleration or slowing down of the incident electron through the electric field of the nucleus, which then emits bremsstrahlung radiation in the form of a photon. The loss of energy in the incident electron is characterized by the total stopping power, $S_{t o t}$. $S_{t o t}$ is the average energy loss per unit distance of a charged particle as it traverses some medium $m$. Like the massenergy absorption coefficient, stopping power tends to be presented as a ratio with medium mass density, or mass stopping power, $\left(\frac{S}{\rho}\right)_{m}$. Figure 1.3 shows the ratio of stopping power in various eye tissues to that in water, $\left(\frac{S}{\rho}\right)_{\text {water }}^{m}$. While the differences seen in $\left(\frac{S}{\rho}\right)_{\text {water }}^{m}$ are not as large as those seen in $\left(\frac{\mu_{e n}}{\rho}\right)_{\text {water }}^{m}$, their existence does give motivation to check the effects the inclusion of eye tissues has on beta plaque dose calculations. 


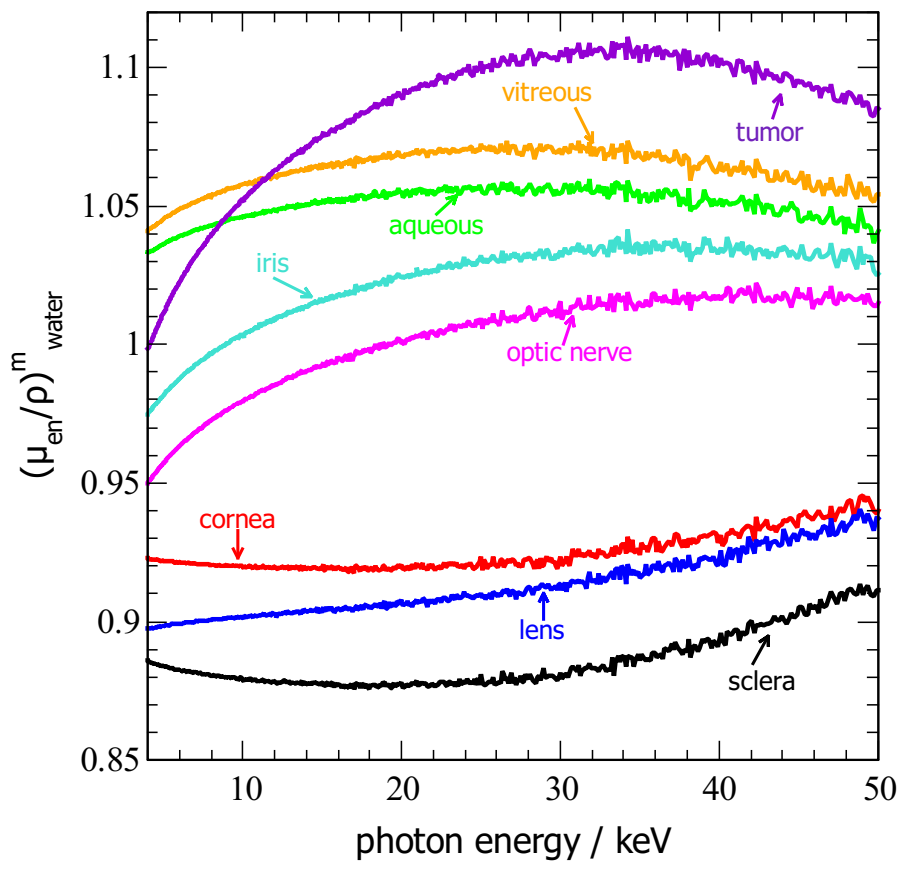

Figure 1.2: Ratios of mass-energy absorption coefficient in different eye tissues to mass-energy absorption coefficient in water at increasing photon energies calculated using the EGSnrc application ' $\mathrm{g}$ '. 


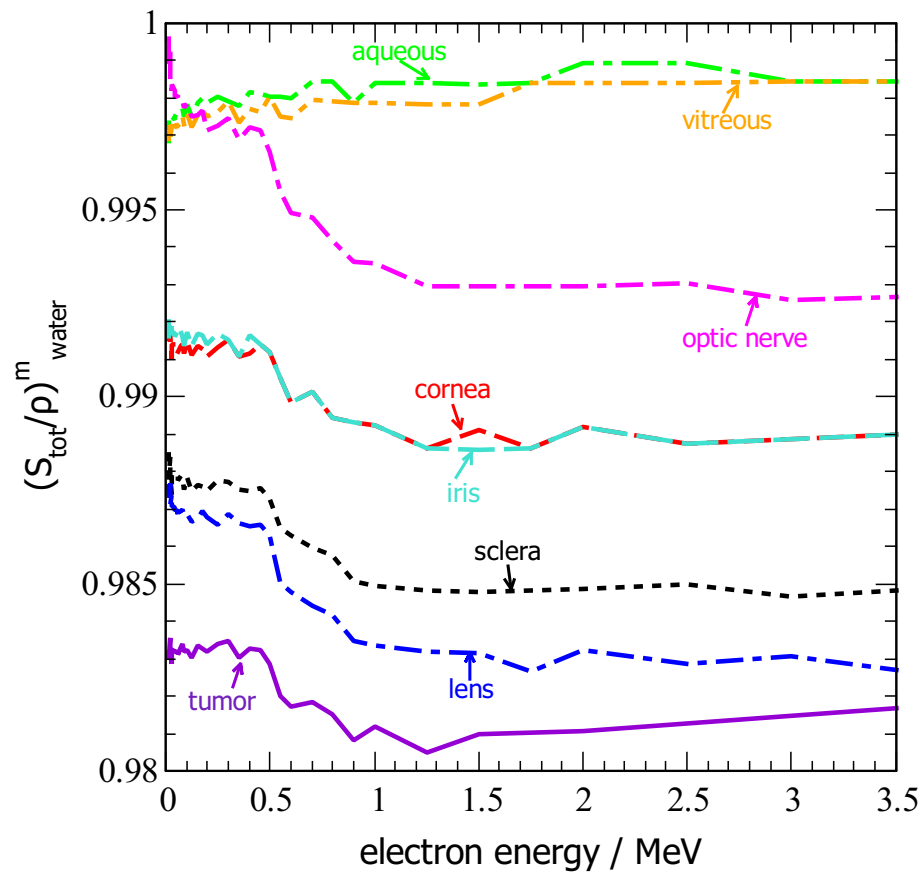

Figure 1.3: Ratios of total electron stopping power in different eye tissues to total electron stopping power in water at increasing electron energies. Stopping powers are calculated using NIST ESTAR ${ }^{39}$. 


\subsection{Model-based and Monte Carlo dose calculations}

Model-based dose calculation algorithms (MBDCAs) are a class of advanced dose calculation algorithms. MBDCAs take into account detailed models of shielding, applicators, seeds, and human tissue when calculating dose. Of the model-based methods that have been published, one of the most promising and the focus of this work is dose calculation through Monte Carlo simulation.

Monte Carlo dose calculations utilize detailed geometries of applicators and sources which are placed into voxelized patient models (where voxels are volume elements). The voxels are assigned compositions and mass densities to create an accurate model of the treatment area. A variety of Monte Carlo codes are capable of calculating eye plaque brachytherapy doses including BrachyDose ${ }^{40,41,42,43}$, the MCNP series of codes ${ }^{44,24,45,42}$, PENELOPE ${ }^{46,47}$, and GEANT4 ${ }^{48,49}$ and many results are available in the literature from these codes.

Recent simulation studies including those by Melhus and Rivard ${ }^{24}$ using MCNP5, and Thomson et al. ${ }^{41,50}$ using BrachyDose give dose results using fully loaded plaques in water. These results found a reduction of dose at tumor apex (depth $5 \mathrm{~mm}$ ) of up to $11 \%$ for ${ }^{125} \mathrm{I}$ seeds and $19 \%$ for ${ }^{103} \mathrm{Pd}$ seeds when compared to calculations emulating the TG43 approach. Further, Rivard et al. ${ }^{42}$ gave dose results from three different brachytherapy TPSs (Pinnacle, BrachyVision, and Plaque Simulator) and two different MC codes (MCNP5 and BrachyDose) for a modelled fully loaded COMS $16 \mathrm{~mm}$ plaque in water and compared them to calculations emulating the TG43 approach. This comparison found that when aiming for a prescription dose of 85 Gy the actual delivery is 76 Gy for ${ }^{125} \mathrm{I}$ seeds and 67 Gy for ${ }^{103} \mathrm{Pd}$ seeds.

Other simulation studies have shown that the use of modified COMS plaques or non-standard plaques creates dose differences as well. Using Plaque Simulator, 
Astrahan et al. ${ }^{51}$ showed differences of up to $11 \%$ when comparing a COMS plaque with a gold alloy seed guide to the traditional Silastic insert. A 2014 work by Lesperance et al. ${ }^{52}$ developed five non-standard plaque models for use in BrachyDose and found significant dose differences not only when compared to TG43 emulating calculations, but also between the plaque models (up to $12 \%$ at the opposite side of the eye). Another 2014 work by Lesperance et al. ${ }^{53}$ utilized an anatomically realistic eye model along with plaque models to calculate doses with BrachyDose. The work found considerable differences when comparing doses calculated with the eye model to those with a plaque in water, signaling a need to advance model-based calculations even further.

This work utilizes the Monte Carlo code, egs_brachy. egs_brachy is a new open source, fast, and efficient Monte Carlo code created specifically for brachytherapy dose calculations by members of the Carleton Laboratory for Radiotherapy Physics (CLRP) research group ${ }^{54}$ and implemented within the EGSnrc family of radiation transport tools. egs_brachy is paired with the open-source egs ++ geometry package, which is used to create models of sources, plaques, and patients.

\subsection{Thesis purpose}

With the need for model-based calculations in treatment planning comes a need for methods to do those calculations. This thesis aims to demonstrate the need for modelling both the plaque and the eye for both photon and beta plaques. The literature does not show any previous attempts to investigate the impact of eye tissues on beta plaque dose distribution compared to water based calculations. This work also aims to verify egs_brachy and plaque models developed in this work as tools for model-based calculations against previously published results. In this process a dataset of eye plaque geometries and dose distributions is developed that will be 
distributed with egs_brachy and on an internet database. This distribution will facilitate future research into eye plaque brachytherapy and the use of model-based calculations for clinical dose evaluation. The created geometries include all sizes of COMS plaques, four models based on non-standard photons plaques, most of the standard BEBIG beta plaque catalog, and a method for creating a more accurate model for BEBIG beta plaques which contain a cutout notch. The development of plaques and the results calculated herein enable a comparison between photon and beta plaque dose distributions and their doses to various eye structures.

In summary, the context and purpose of this thesis are as follows: Current clinical approach to dose calculations has been found inadequate. The TG43 formalism does not accurately emulate the physics behind photon plaque brachytherapy, nor does it compare favourably to MBDCA results found in the literature. Further, a standardized calculation method for beta plaque brachytherapy does not even exist. The work done in this thesis aims to contribute to the advancement of dose evaluation for treatment and research through the use of Monte Carlo calculations. The purposes of this thesis are to:

(i) Demonstrate the need for model-based dose calculations

(ii) Create a database of geometries and dose distributions for eye plaque brachytherapy

(iii) Verify egs_brachy dose distributions against other published results

(iv) Compare dose distributions of photon and beta plaques 


\subsection{Thesis outline}

Chapter 1 has introduced the ideas behind eye cancer treatment and current dose evaluation, as well as why model-based calculations are being investigated. Next, Chapter 2 explores the methods used to model the source, plaque, and phantom geometries and obtains accurate dose calculations from the Monte Carlo simulations using those geometries. Chapter 3 reports the results of these simulations and verification for photon plaques, then beta plaques, and finally a comparison between the two. Chapter 4 discusses the verification of egs_brachy and the plaque models, the comparison of model based calculations to those which emulate the TG43 protocol, and the differences between photon and beta dose distributions. Chapter 5 discusses conclusions drawn from the results of the thesis as well as future work that could be enabled by it. Lastly, an Appendix includes information on the seed positions for all photon plaques presented in this work. 


\section{Chapter 2}

\section{Methods}

This chapter describes the methods used to create eye plaque dose distributions with egs_brachy version v2017.09.15. The chapter starts with the development of the geometries used for this work including the eye plaque applicator, seed sources, phantoms, and scoring regions. Following an introduction to the geometries is a summary of the parameters used for simulations and the methods for calculation and normalization of dose. Finally, a description of the steps taken to verify the doses against other Monte Carlo codes is included.

Two Cartesian coordinate systems are used when discussing the plaque and phantom geometries, as well as dose distributions: the eye plaque coordinate system and the eye centre coordinate system. The former has an origin located at the inner sclera on the $\mathrm{z}$ axis and the centre of the plaque face on the xy plane, and the latter has an origin located at the centre of the eye. These coordinate systems are based on those used in the Collaborative Ocular Melanoma Study (COMS) where the eye was assumed to have a diameter of $2.46 \mathrm{~cm}$ and a sclera $1 \mathrm{~mm}$ thick. The eye plaque coordinate system can be converted to the eye centre coordinate system by a translation of $+1.13 \mathrm{~cm}$ along the $\mathrm{z}$ axis. To simplify notation the eye plaque coordinate system will use the variables $(x, y, z)$ and the eye centre coordinate system (X, Y, Z). Figure 2.1 shows the location of the two coordinate systems. 
(a)

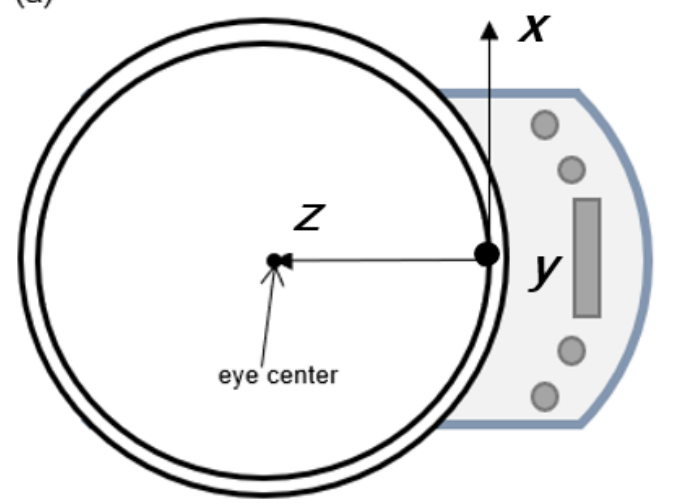

(b)

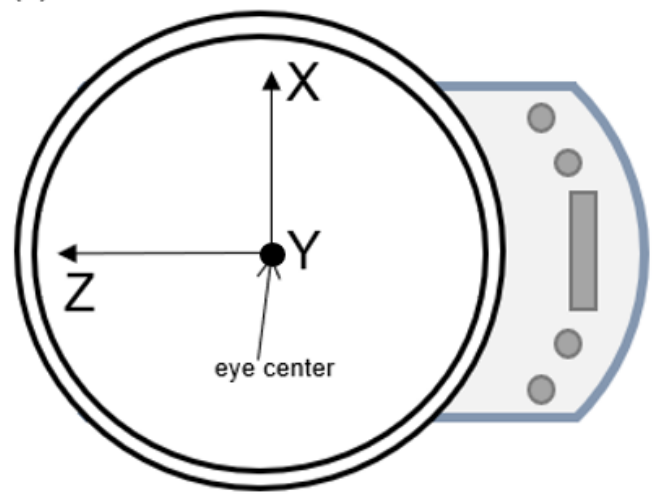

Figure 2.1: Schematic diagram of eye coordinate systems (not to scale), (a) shows the eye plaque coordinate system $(x, y, z)$ with the origin at the inner sclera; $(b)$ shows the eye centre coordinate system (X, Y, Z) where the origin is at the centre of the eye. A shift of $z+1.13 \mathrm{~cm}$ to convert from $(x, y, z)$ to $(\mathrm{X}, \mathrm{Y}, \mathrm{Z})$ is needed. In both cases $y / Y$ is coming out of the page.

\subsection{Eye plaque model geometries}

egs_brachy utilizes the open source egs ++ geometry package for the creation of simulation geometries. This work involves the creation of many different plaque (both photon and beta) models with egs ++ . The models will be distributed through an internet database and eventually included in a future version of egs_brachy. This section includes the geometric descriptions of 10 to $24 \mathrm{~mm}$ COMS, "no lip - silastic", "short lip - acrylic", "COMS thin - acrylic", "stainless steel - acrylic", CCA, CCB, CCC, CCD, CXS, CCX-Y-Z, CGD, and CIA plaques. Each of these plaque models (with CCB representing the beta plaques) can be seen placed on the nasal side of a model of the right eye in Figure 2.2. 


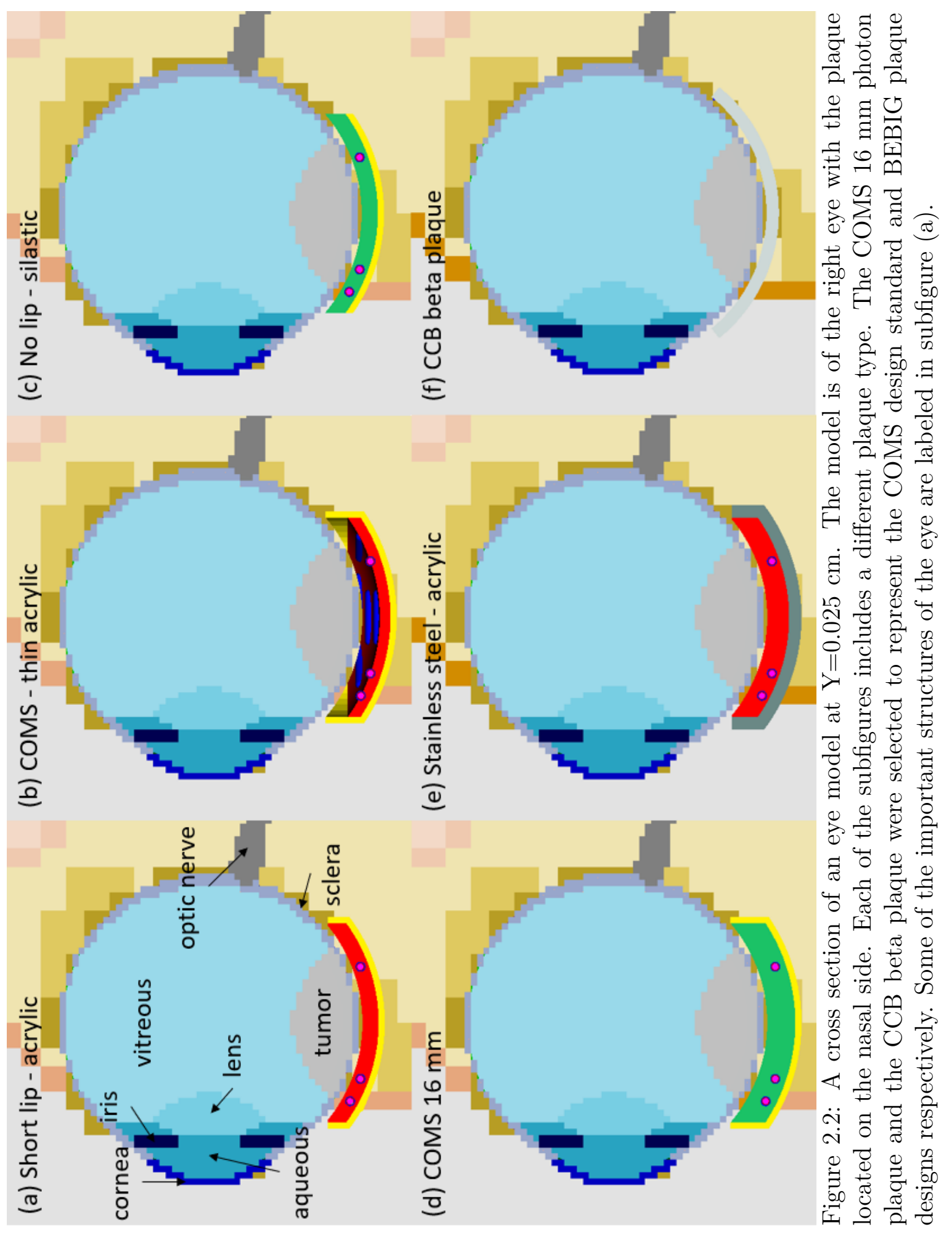




\subsubsection{Photon plaque geometries}

\section{Standard COMS plaques}

In 1985 the Collaborative Melanoma Study (COMS) created a collection of standardized eye plaques. These eye plaques consist of a gold alloy backing and a siliconepolymer insert. Commercially, these materials are known as Modulay and Silastic respectively. For the backing a medium composed of gold, silver, copper, and palladium (mass fraction composition is $0.77,0.14,0.08$, and 0.01 respectively) with a mass density $\rho$ of $15.8 \mathrm{~g} \mathrm{~cm}^{-3}$ is used. For the insert the medium is given a composition of hydrogen, carbon, oxygen, silicon, and platinum (mass fraction composition is $0.063,0.249,0.289,0.399$, and 0.00005 respectively) with $\rho=1.12 \mathrm{~g} \mathrm{~cm}^{-3}$. The radioactive source for these eye plaques consists of standard brachytherapy seeds that are fitted into the Silastic insert. Each plaque size has a different set of positional indentations in the insert for fitting the seeds (e.g. the $16 \mathrm{~mm}$ diameter COMS plaque has indentations for 13 seeds within its insert). Seed positions for COMS 10

mm to COMS $22 \mathrm{~mm}$ are taken from the AAPM Task Group 129 report $^{36}$ and are included in Appendix A. For more detailed information on seed positions refer to Table A.1, and Figures 2.3 and 2.4. The original set of eye plaques ranged from 12 $\mathrm{mm}$ to $20 \mathrm{~mm}$ in diameter in $2 \mathrm{~mm}$ increments. The $10 \mathrm{~mm}, 22 \mathrm{~mm}$, and $24 \mathrm{~mm}^{55}$ plaques were added to the range in the following years.

The plaque designation (e.g. COMS $16 \mathrm{~mm}$ ) gives the diameter of the plaque insert at its widest point in the $x y$ plane. The backing adds $1 \mathrm{~mm}$ to the overall diameter (e.g. COMS $16 \mathrm{~mm}$ is $17 \mathrm{~mm}$ in total diameter). The inner and outer radius of curvature for the backing are $14.55 \mathrm{~mm}$ and $15.05 \mathrm{~mm}$ respectively for all plaque sizes. The radius of curvature of the insert where it rests against the eye is 


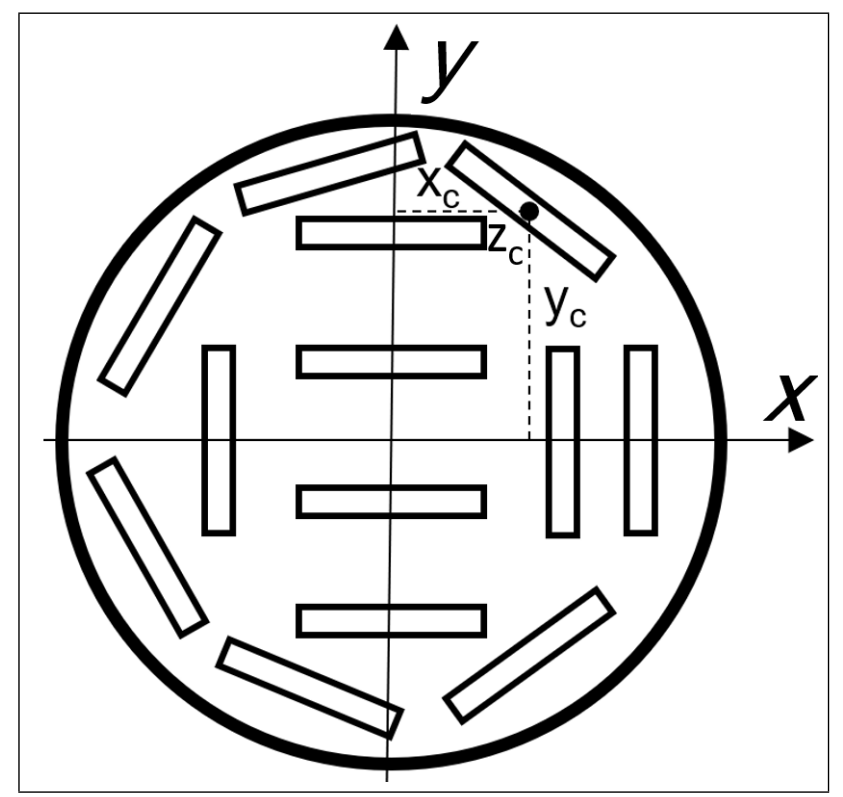

Figure 2.3: Schematic diagram of COMS plaque seed positions (not to scale). Seed positions are for a COMS $16 \mathrm{~mm}$ plaque. $x_{c}, y_{c}$ and $z_{c}$ are the $x, y$, and $z$ coordinates for the seed centres, where $z$ comes out of the page.

$12.30 \mathrm{~mm}$, once again for all plaque sizes. Each plaque size has a different height, which is given in Table 2.1 .

For the purposes of modelling the COMS plaques, they are divided up into three 'regions' as shown in Figure 2.5. Region 1 consists of curved backing on both the inside and the outside. Region 2 is a small region where the inside of the plaque is still curved but the outside has become a straight lip. For Region 3 the backing is all lip, while the insert ends in the curved indentation meant to sit against the eye.

The COMS $24 \mathrm{~mm}$ plaque was not a part of the original study and was developed by McCauley Custinger et al. ${ }^{56}$ for the Mayo Clinic in 2015. It was created to treat larger tumors than treatable by available COMS plaques. Geometry information can be found in Table 2.1 and seed positions in Table A.1. Not all plaques used in the 


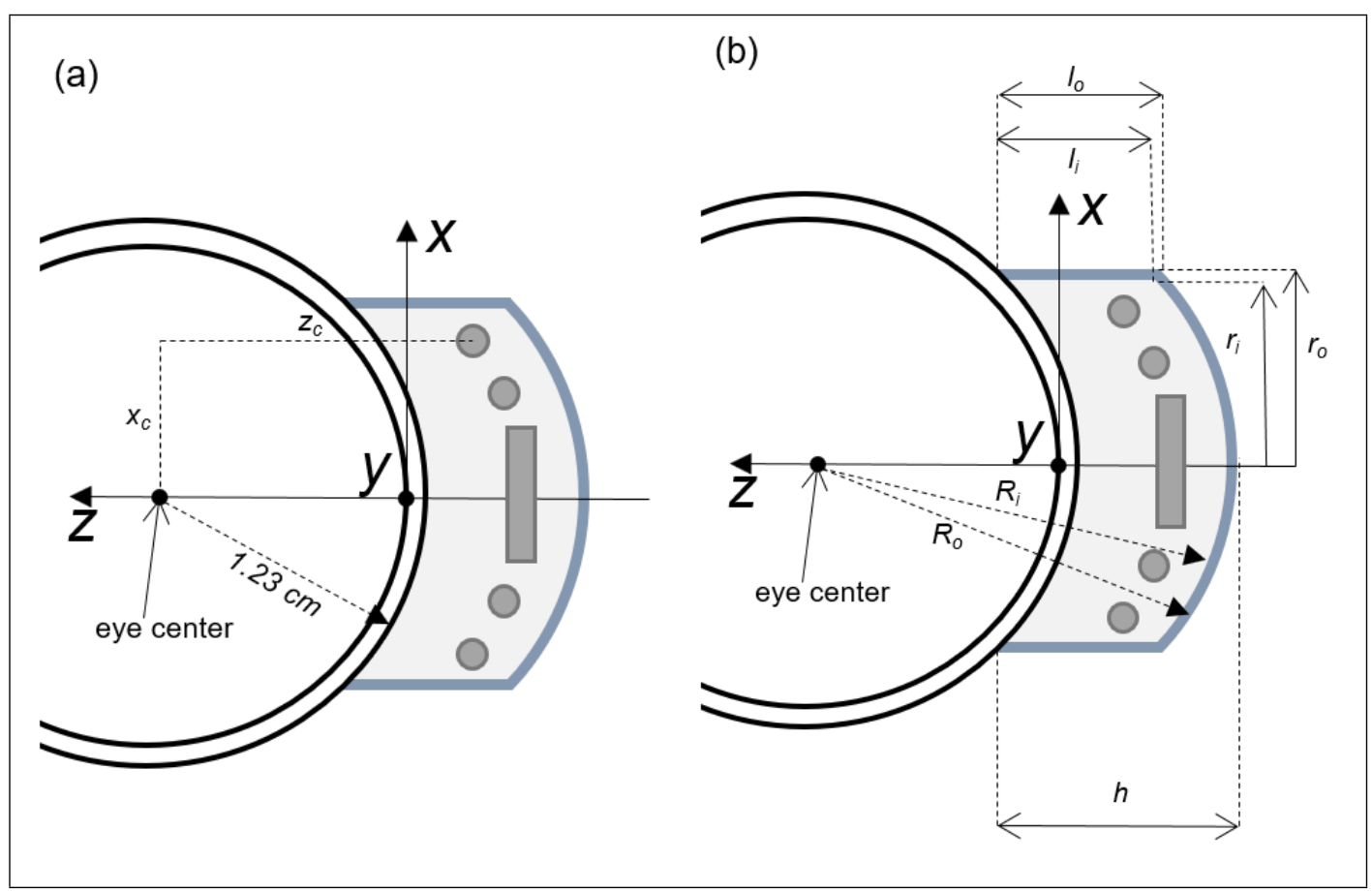

Figure 2.4: Schematic diagrams of COMS plaque geometry parameters (not to scale). Subfigure (a) shows the eye plaque coordinate system where the z-axis is along the plaque central axis and the origin is at the point where the z-axis intersects with the inner sclera. $x_{c}, y_{c}$ and $z_{c}$ are the $x, y$, and $z$ coordinates for the seed centres, where the $y$-axis is out of the page. Subfigure (b) shows the geometry parameters of the plaque; $R_{o}$ is the outer radius of curvature of the plaque backing; $R_{i}$ is the inner radius of curvature of the plaque backing; $r_{i}$ is the inner radius of the plaque backing; $r_{o}$ is the outer radius of the plaque backing; $l_{i}$ is the inner lip length of the plaque backing; $l_{o}$ is the outer lip length of the plaque backing; and $h$ is the total height of the plaque. 


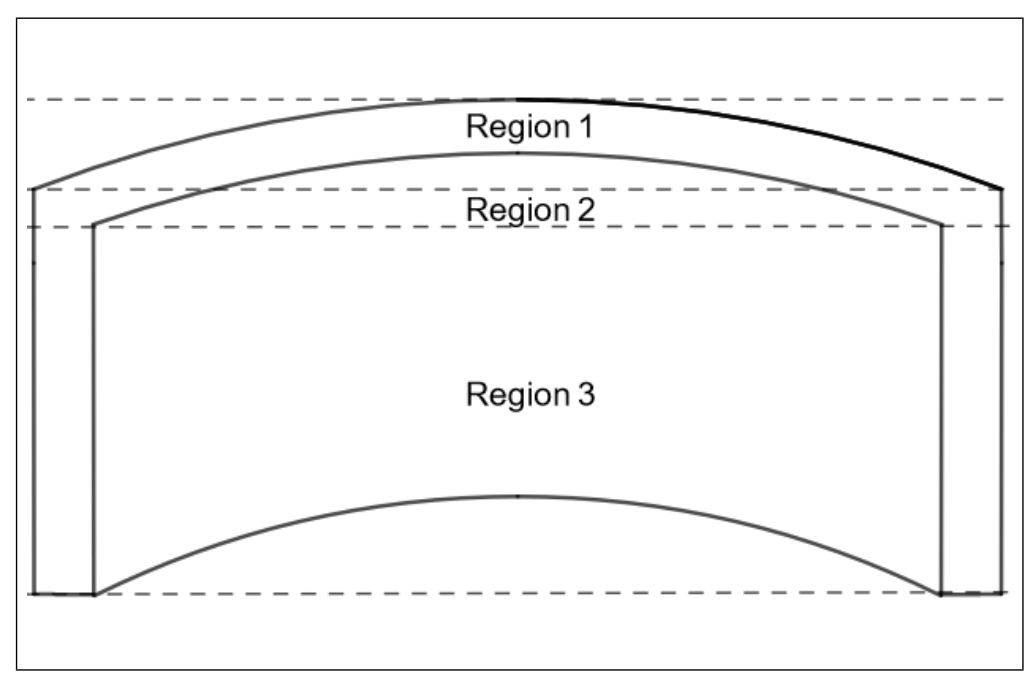

Figure 2.5: Schematic diagram of the three regions which make up the COMS plaque geometry in egs ++ (not to scale). Region 1 has wholly curved backing, Region 2 has curved inner backing and straight outer backing, and Region 3 has both inner and outer backing that are straight and includes the indent placed against the eye.

clinic follow the COMS plaque guidelines perfectly and often use different materials or sizes than the standard. Four plaque models, described below, based on those found in a paper by Lesperance et. al. are provided as an example of other potential models ${ }^{52}$.

\section{"Short lip - acrylic"}

This plaque has a shorter lip than the standard COMS $16 \mathrm{~mm}$ plaque. While the backing is still Modulay, the insert is acrylic (all acrylic inserts are given a mass fraction composition of 0.6 carbon, 0.32 oxygen, and 0.08 hydrogen, and mass density $\left.1.19 \mathrm{~g} \mathrm{~cm}^{-3}\right)$. The short lip causes the seed centres to be closer to the eye with $r_{\text {seed }}=1.295 \mathrm{~cm}$. More detailed information on seed positions is available in Table A.1, and for the plaque geometry in Table 2.1. Figure 2.2 gives a visual representation 
of this plaque in a patient model.

\section{"COMS - thin acrylic"}

This plaque still has a Modulay backing, but has a thin acrylic insert that the seeds are attached to, rather than inserted into. It was designed to approximate plaques like those used in a few different experimental studies. In one work seeds were affixed to a thin acrylic insert placed in a standard COMS backing ${ }^{57}$. Another similar plaque uses a thin reusable seed guide to affix the seeds to an acrylic layer in the same positions as the standard COMS plaque ${ }^{51}$. Similarly, the OSU-NAG plaque is a

Modulay backed plaque with fewer seeds that are also glued onto the backing ${ }^{58}$. The plaque in this work is modelled with the same seed positions as the standard COMS $16 \mathrm{~mm}$ plaque. More detailed information on seed positions is available in Table A.1 and for the plaque geometry in Table 2.1. Figure 2.2 gives a visual representation of this plaque in a patient model.

\section{"No lip - Silastic"}

This plaque still has a Modulay backing with the standard Silastic insert, but the backing has no collimating lip. Plaques used by both Puusaari et al. ${ }^{59}$ and Nag et al. ${ }^{60}$ use a gold backing with no collimating lip, though the latter affixes the seeds directly to the backing. For the plaque model presented here the backing is Modulay like the other COMS plaques rather than gold. The seeds are placed at the same position as for the short lip plaque with seed centres $r_{\text {seed }}=1.295 \mathrm{~cm}$. More detailed information on seed positions is available in Table A.1 and for the plaque geometry in Table 2.1. Figure 2.2 gives a visual representation of this plaque in a patient model. 
Table 2.1: Geometric dimensions for all photon plaques where $r_{\text {seed }}$ is the radius from eye centre to a seed centre; $R_{o}$ is the outer radius of curvature of the plaque backing; $r_{i}$ is the inner radius of the plaque backing; $r_{o}$ is the outer radius of the plaque backing; $l_{i}$ is the inner lip length of the plaque backing; $l_{o}$ is the outer lip length of the plaque backing; and $\mathrm{h}$ is the total height of the plaque as defined in Figure 2.4.

\begin{tabular}{|c|c|c|c|c|c|c|c|}
\hline Plaque & $\begin{array}{c}r_{\text {seed }} \\
(\mathrm{cm})\end{array}$ & $\begin{array}{c}R_{o} \\
(\mathrm{~cm})\end{array}$ & $\begin{array}{c}r_{i} \\
(\mathrm{~cm})\end{array}$ & $\begin{array}{c}r_{o} \\
(\mathrm{~cm})\end{array}$ & $\mathrm{h}(\mathrm{cm})$ & $l_{i}(\mathrm{~cm})$ & $\begin{array}{c}l_{o} \\
(\mathrm{~cm})\end{array}$ \\
\hline COMS 10 mm & 1.370 & 1.505 & 0.50 & 0.55 & 0.38 & 0.24 & 0.28 \\
\hline COMS 12 mm & 1.370 & 1.505 & 0.60 & 0.65 & 0.43 & 0.25 & 0.28 \\
\hline COMS 14 mm & 1.370 & 1.505 & 0.70 & 0.75 & 0.49 & 0.26 & 0.29 \\
\hline COMS 16 mm & 1.370 & 1.505 & 0.80 & 0.85 & 0.57 & 0.28 & 0.31 \\
\hline COMS 18 mm & 1.370 & 1.505 & 0.90 & 0.95 & 0.67 & 0.30 & 0.33 \\
\hline COMS 20 mm & 1.370 & 1.505 & 1.00 & 1.05 & 0.79 & 0.34 & 0.36 \\
\hline COMS 22 mm & 1.370 & 1.505 & 1.10 & 1.15 & 1.00 & 0.40 & 0.42 \\
\hline COMS 24 mm & 1.370 & 1.505 & 1.20 & 1.25 & 1.23 & 0.55 & 0.57 \\
\hline "Short lip - acrylic" & 1.295 & 1.410 & 0.80 & 0.85 & 0.46 & 0.15 & 0.30 \\
\hline "No lip - silastic" & 1.295 & 1.410 & 0.80 & - & 0.47 & - & - \\
\hline "COMS - thin acrylic" & 1.370 & - & 0.80 & 0.85 & 0.57 & 0.28 & 0.31 \\
\hline "Stainless steel - acrylic" & 1.345 & 1.505 & 0.80 & 0.90 & 0.56 & 0.21 & 0.26 \\
\hline \hline
\end{tabular}

\section{"Stainless steel - acrylic"}

This plaque has a thicker $(1 \mathrm{~mm})$ stainless steel backing (mass fraction elemental composition of 0.9905 iron, 0.015 carbon, 0.05 manganese, and 0.03 sulfur with mass density $7.9 \mathrm{~g} \mathrm{~cm}^{-3}$ ) instead of the standard Modulay, and uses an acrylic insert. This plaque is representative of the ROPES eye plaque ${ }^{61}$ as well as a plaque developed by Karolis et al. ${ }^{62}$ The seed centres are again slightly different from the COMS 16 $\mathrm{mm}$ plaque with $r_{\text {seed }}=1.345 \mathrm{~cm}$. More detailed information on seed positions is available in Table A.1 and for the plaque geometry in Table 2.1. Figure 2.2 gives a visual representation of this plaque in a patient model. 


\section{Source models}

For photon plaques the sources are standard brachytherapy seeds. egs_brachy comes bundled with various seed models created with egs ++ and these are used as the source geometries for the photon plaque simulations in this thesis. Two different seed types are used, an ${ }^{125} \mathrm{I}$ seed model based on the Amershan OncoSeed 6711 seed ${ }^{63,64}$ and a ${ }^{103} \mathrm{Pd}$ seed model based on the Theragenics Co. TheraSeed 200 (T200) seed ${ }^{65,66,67}$. The egs_brachy models of these seeds were benchmarked in Chamberland et al. ${ }^{54}$ in 2016.

The 6711 seed (shown in Figure 2.6) has an outer container of titanium ( $\rho=$ $4.54 \mathrm{~g} \mathrm{~cm}^{-3}$ ) with a length of $3.75 \mathrm{~mm}$, a diameter of $0.800 \mathrm{~mm}$, walls that are $0.0700 \mathrm{~mm}$ thick, and hemi-spherical end caps that are $0.375 \mathrm{~mm}$ thick. Within this titanium tube is a silver $\operatorname{rod}\left(\rho=10.5 \mathrm{~g} \mathrm{~cm}^{-3}\right)$ of length $2.80 \mathrm{~mm}$, a radius of $0.250 \mathrm{~mm}$, and end points have radius $0.175 \mathrm{~mm}$. This silver rod is coated in a $1.75 \mu \mathrm{m}$ thick $\mathrm{AgI} / \mathrm{AgBr}$ isotope (elemental composition 0.536 Ag, $0.284 \mathrm{Br}, 0.18 \mathrm{I}$, and $\rho=6.20 \mathrm{~g} \mathrm{~cm}^{-3}$ ). The coated silver rod is left free to move within the titanium cylinder ${ }^{64}$. Table 2.2 gives the tabulated line spectrum used to initialize photons in the ${ }^{125}$ I seeds ${ }^{68,69}$.

Table 2.2: Spectrum used for ${ }^{125} I$ brachytherapy seeds taken from NCRP Report $58^{68,69}$.

\begin{tabular}{|c|c|}
\hline $\begin{array}{c}\text { Energy } \\
(\mathrm{MeV})\end{array}$ & $\begin{array}{c}\text { Intensity } \\
(\%)\end{array}$ \\
\hline 0.0272017 & 0.397 \\
0.0274723 & 0.741 \\
0.031 & 0.257 \\
0.0354919 & 0.0668 \\
\hline \hline
\end{tabular}

The T200 seed shown in Figure 2.6 has a more complex geometry than the 6711 seed. The outer container is a titanium tube with wall thickness $0.0560 \mathrm{~mm}$ 
and outer diameter $0.826 \mathrm{~mm}$. This tube has end cups rather than end caps that have a thickness of $0.040 \mathrm{~mm}$. The end cups are a hemispherical shell with a hollow cylinder of length $0.334 \mathrm{~mm}$ attached, and both have an inner radius $0.306 \mathrm{~mm}$. The combination of tube and end cups has a length of $4.500 \mathrm{~mm}$. Within the tube are two graphite cylinders $\left(\rho=2.265 \mathrm{~g} \mathrm{~cm}^{-3}\right)$ coated in radioactive $\mathrm{Pd}(\rho=12.02$ $\left.\mathrm{g} \mathrm{cm}^{-3}\right)$. The two cylinders are separated by a lead cylinder $66\left(\rho=11.34 \mathrm{~g} \mathrm{~cm}^{-3}\right)$. Refer to Table 2.3 for the tabulated line spectrum used to intialize photons in the ${ }^{103} \mathrm{Pd}$ seeds ${ }^{69,70}$.

Table 2.3: Spectrum used for ${ }^{103} P d$ brachytherapy seeds taken from NNDC $2.6^{70,69}$.

\begin{tabular}{|c|c|}
\hline $\begin{array}{c}\text { Energy } \\
(\mathrm{MeV})\end{array}$ & $\begin{array}{c}\text { Intensity } \\
(\%)\end{array}$ \\
\hline 0.002700 & 0.0873 \\
0.020074 & 0.2240 \\
0.020216 & 0.4250 \\
0.022699 & 0.0354 \\
0.022724 & 0.0685 \\
0.023172 & 0.0164 \\
0.039748 & $6.83 \mathrm{E}-4$ \\
0.053290 & $3.00 \mathrm{E}-7$ \\
0.062410 & $1.04 \mathrm{E}-5$ \\
0.241880 & $5.00 \mathrm{E}-9$ \\
0.294980 & $2.80 \mathrm{E}-5$ \\
0.357450 & $2.21 \mathrm{E}-4$ \\
0.443790 & $1.50 \mathrm{E}-7$ \\
0.497080 & $3.96 \mathrm{E}-5$ \\
\hline \hline
\end{tabular}

\subsubsection{Beta plaque geometries}

The ${ }^{106} \mathrm{Ru} /{ }^{106} \mathrm{Rh}$ beta plaque geometries are all bowl-shaped plaques made of silver. A very thin layer $(\sim 0.1 \mu \mathrm{m})$ of the active source coats the plaque under a $0.1 \mathrm{~mm}$ silver window. The plaque itself is modelled in egs ++ by using a cone of the appropriate opening angle to cut it out from a silver shell $\left(\rho=10.5 \mathrm{~g} \mathrm{~cm}^{-3}\right)$. The angles 
(a)

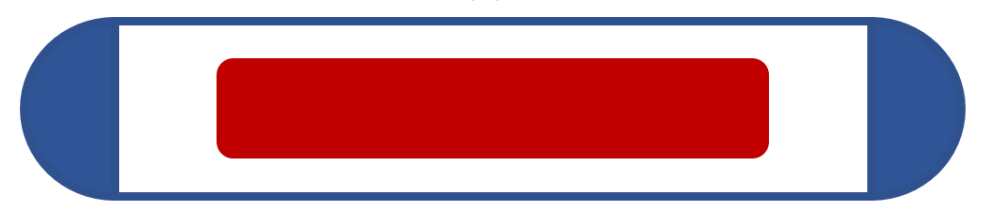

(b)

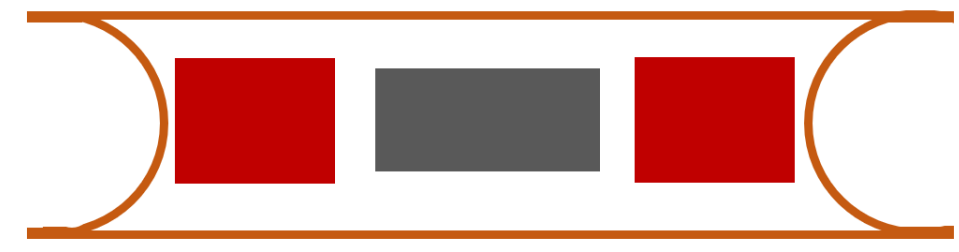

Figure 2.6: Geometry of brachytherapy seeds used in this work. Subfigure(a) shows Amershan OncoSeed $6711^{125} \mathrm{I}$ seed; blue is titanium casing and red is silver rod coated in radioisotope. Subfigure (b) shows Theragenics Co. TheraSeed $200{ }^{103} \mathrm{Pd}$ seed; orange is the titanium casing, grey is lead cylinder, red are graphite cylinders coated in radioisotope.

used for each plaque type as well as their radii and other geometric information can be found in Table 2.4. Figure 2.7 is a schematic diagram that shows how the plaques are constructed given the information in Table 2.4, while Figure 2.2 gives a visual representation of the $\mathrm{CCB}$ plaque in a patient model.

For the beta plaques the source is modelled using a radionuclide source model (known as the EGS_RadionuclideSource). The radionuclide model uses Evaluated Nuclear Structure Data Files (ENSDFs) from the Laboratoire National Henri Becquerel (LNHB) to model the full decay spectrum of a nuclide. 


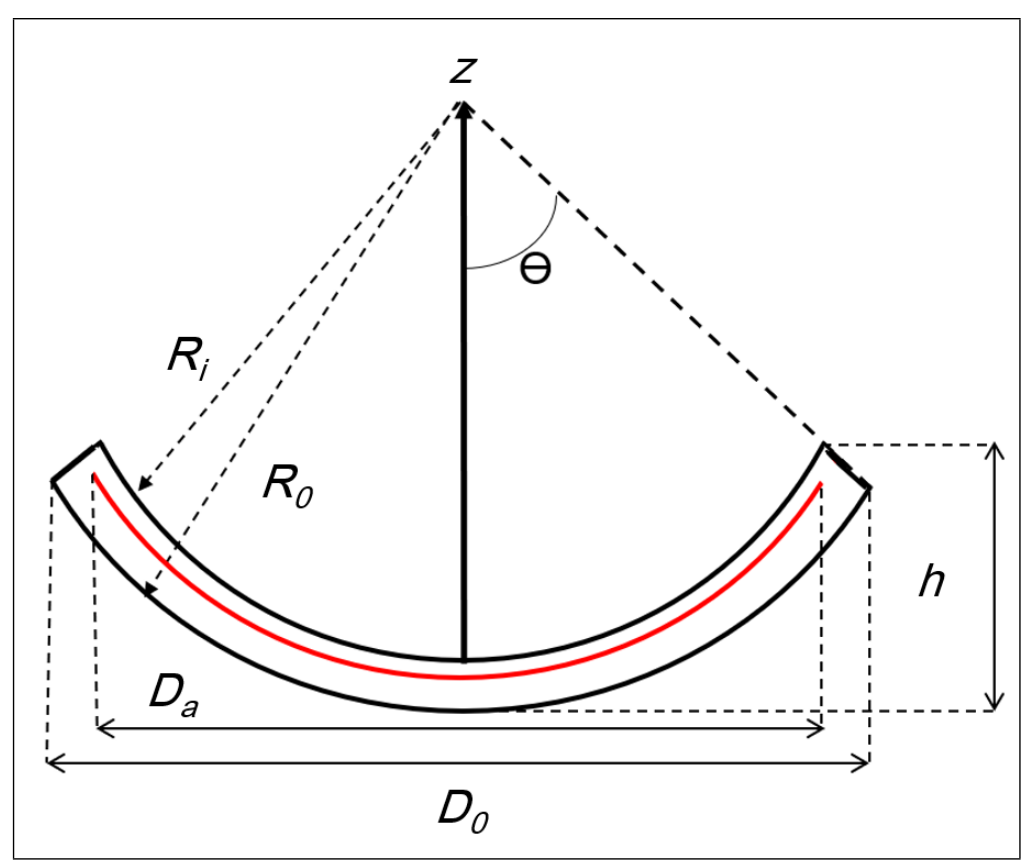

Figure 2.7: Schematic diagram showing geometry parameters for BEBIG beta plaques (not to scale). $R_{i}$ and $R_{o}$ are the radii of curvature for the inner and outer plaque; $D_{a}$ is the diameter of the active source; $D_{o}$ is the diameter of the full plaque; $\mathrm{h}$ is the height of plaque; and the opening angle $\theta$ is the angle used to cut the plaque from a spherical shell.

Table 2.4: Geometric dimensions for all beta plaques. $R_{i}$ and $R_{o}$ are the radii of curvatures for the inner and outer plaque; $r_{i}$ is the radius of the active source; $r_{o}$ is the radius of the full plaque; $h$ is the height of plaque; and the opening angle $\theta$ is the angle used to cut the plaque from a spherical shell.

\begin{tabular}{|c|c|c|c|c|c|c|}
\hline Plaque type & $R_{i}(\mathrm{~cm})$ & $R_{o}(\mathrm{~cm})$ & $D_{a}(\mathrm{~cm})$ & $D_{o}(\mathrm{~cm})$ & $\mathrm{h}(\mathrm{cm})$ & $\theta\left(^{\circ}\right)$ \\
\hline CCA & 1.20 & 1.30 & 1.30 & 1.53 & 0.33 & 36.04 \\
\hline CCB & 1.20 & 1.30 & 1.78 & 2.02 & 0.54 & 50.82 \\
\hline CCC & 1.30 & 1.40 & 2.25 & 2.48 & 0.80 & 62.34 \\
\hline CCD & 1.20 & 1.30 & 1.55 & 1.79 & 0.43 & 43.51 \\
\hline CGD & 1.30 & 1.40 & 1.99 & 2.23 & 0.61 & 52.79 \\
\hline CCX-Y-Z & 1.20 & 1.30 & 0.95 & 1.16 & 0.23 & 26.49 \\
\hline CXS & 1.20 & 1.30 & 0.77 & 1.16 & 0.23 & 26.49 \\
\hline \hline
\end{tabular}




\section{Two versions of beta plaque with cutout notch}

Beta plaques that contain a cutout notch are more complex and require a slightly different approach. The base plaque is designed in the same way as for the symmetric plaques (e.g. the plaque presented here, the CIA plaque, has the same base dimensions as the CCA symmetric plaque seen in Table 2.1). Two different methods are implemented to create the cutout.

The first emulates the work of Hermida-Lopez ${ }^{47}$ (with geometrical values gained from personal communication, Hermida-Lopez 2018) and uses a bounding cylinder geometry to fill the cutout portion of the plaque with water. The cylinder is of radius $0.7 \mathrm{~cm}$ and located at a midpoint of $(0.915,0,-1.2) \mathrm{cm}$ in eye centre coordinates. The sharp corners of the plaque left by the cylinder are then cutoff with a clipping plane at $0.43 \mathrm{~cm}$ on the x-axis. This results in the geometry of Figure 2.8(a). This version of the CIA plaque will henceforth be referred to as the CIA-HL model in this thesis.

The second method uses the egs++ geometry package to round the 'corners' rather than clipping them off. This is done by splitting the plaque into two regions at $0.32 \mathrm{~cm}$ along the $\mathrm{x}$-axis. One region, which contains the majority of the plaque, uses the same bounding cylinder from the first method to create a notch. The other smaller region uses two small cylinders to cut off the area around the 'corners'. These cylinders are $0.175 \mathrm{~cm}$ in radius and are located at $(0.32,0.55,-1.2)$ and $(0.32,-0.55$, -1.2) cm in eye centre coordinates. The resultant model can be seen in Figure 2.8(b). The second version will henceforth be referred to as the CIA model. 


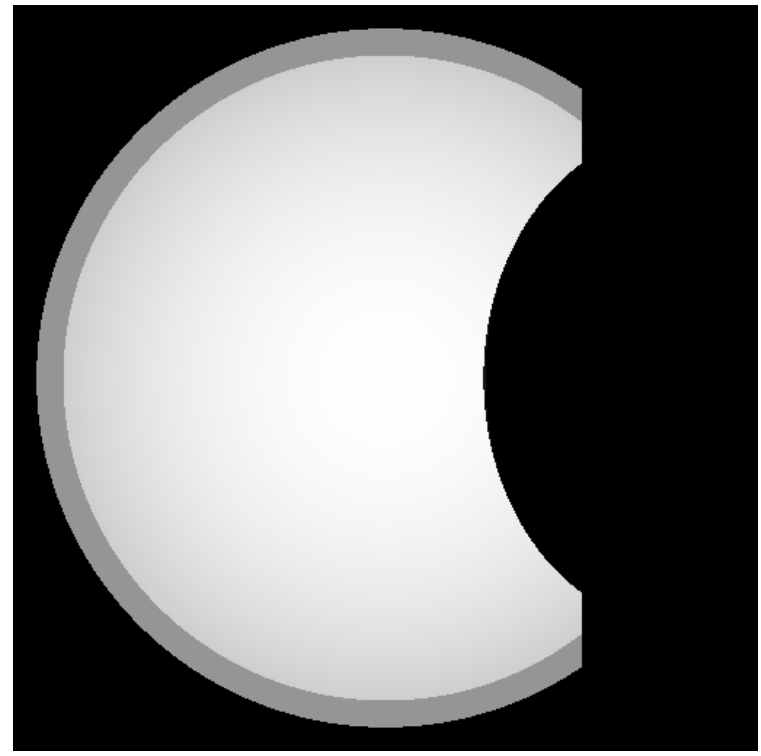

(a)

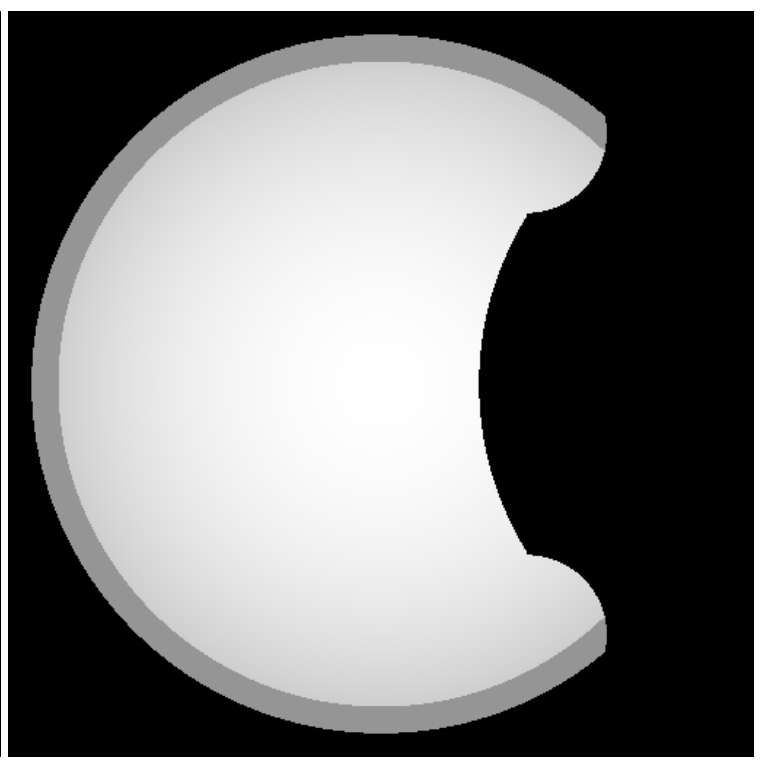

(b)

Figure 2.8: (a) CIA-HL plaque model with points of notch clipped off; (b) CIA plaque model with points of notch rounded out.

\subsection{Phantom geometry}

All simulations in this work are done within a 'phantom,' which is a representation of the patient created by assigning media to different regions of a geometry in which radiation will be transported. For the simulations in this work the phantom is either a $(30 \times 30 \times 30) \mathrm{cm}^{3}$ cube of water centered at $(0,0,0) \mathrm{cm}$ or a more realisitic representation of the eye detailed in Section 2.2.1. The phantom contains a dose scoring grid of voxels (volume elements) where the dose is calculated, the region outside of this scoring region is made much larger than the scoring region to allow scattered particles to return to the scoring region. The dimensions and locations within the eye coordinate system of scoring regions used in this work can be seen in Table 2.5. All water in this work is given $\rho=0.998 \mathrm{~g} \mathrm{~cm}^{-3}$ as recommended in the TG43 protocol $^{34}$. 
Table 2.5: Dimensions of voxelized scoring regions for simulations scoring in water $\left(D_{T G 43}\right.$ and $\left.D_{w, w}\right)$.

\begin{tabular}{|c|c|c|c|c|c|c|c|}
\hline $\begin{array}{c}\text { Source } \\
\text { type }\end{array}$ & $x_{\min }(\mathrm{cm})$ & $x_{\max }(\mathrm{cm})$ & $y_{\min }(\mathrm{cm})$ & $y_{\max }(\mathrm{cm})$ & $z_{\min }(\mathrm{cm})$ & $z_{\max }(\mathrm{cm})$ & $\begin{array}{c}\text { voxel } \\
\text { vol. } \\
\left(\mathrm{cm}^{3}\right)\end{array}$ \\
\hline Photon & -1.275 & 1.275 & -1.275 & 1.275 & -0.075 & 2.475 & 0.000125 \\
\hline Beta & -1.575 & 1.575 & -1.575 & 1.575 & 0.025 & 3.175 & 0.000125 \\
\hline \hline
\end{tabular}

\subsubsection{Voxelized eye phantom model}

Lesperance et al. ${ }^{53}$ created a voxelized eye phantom model for use with BrachyDose in 2014. The model was converted to the .egsphant format, which egs_brachy can utilize for more accurate dose calculations. The composition and dimensions of structures within the eye vary based on several factors such as age and existing eye conditions, so the eye phantom uses averaged dimensions and compositions for ocular structures to create a realistic model of a human eye (specifically the right eye). The eye model is inserted into an ICRP reference computational phantom of a human head $^{71}$ and a coordinate system is created such that the eye centre is at the origin $(0$, $0,0) \mathrm{cm}$. The structures modelled include the sclera, the cornea, the iris, the lens, the aqueous humor, the vitreous humor, the optic nerve, and a nasal uveal tumor.

The sclera is modelled as a sphere that has an outer diameter of $24 \mathrm{~mm}^{72,73,74}$. The inner diameter varies as an ellipsoid where the sclera has a thickness of $0.3 \mathrm{~mm}$ on the plane between anterior and posterior halves of the eye and a thickness of 1 $\mathrm{mm}$ at the anterior and posterior poles ${ }^{72}$. The cornea is the region between two spheres of radii $7.8 \mathrm{~mm}$ and $6.5 \mathrm{~mm}^{72}$. The outer cornea intersects with the outer sclera to form a circle with radius $5.85 \mathrm{~mm}^{72}$. The thickness of the cornea is $0.52 \mathrm{~mm}$ where both the inner cornea and outer cornea intersect with the $\mathrm{x}$-axis ${ }^{72}$. The iris is the region between two cylinders of diameters 5.0 and $11.7 \mathrm{~mm}^{73}$. These cylinders 
are cut off by two planes to give the iris a thickness of $0.6 \mathrm{~mm}$ in the $x$ direction ${ }^{73}$. The anterior surface of the iris rests at the intersection between the cornea and the inner sclera. The lens region is formed from two spheres of radii $6 \mathrm{~mm}$ and $10 \mathrm{~mm}$ for the posterior and anterior surfaces respectively ${ }^{72}$. The intersection of the two spheres gives the lens a diameter of $9.5 \mathrm{~mm}$ in the yz plane at its widest point ${ }^{72}$. The aqueous humor is contained within the region between the inner cornea and the anterior of the lens. The vitreous humor takes up the rest of the eye within the inner sclera. The optic nerve is modelled as three cylindrical segments that increase from $1.5 \mathrm{~mm}$ in diameter at the optic disc to $2.5 \mathrm{~mm}$ in mid-sclera, and $3.5 \mathrm{~mm}$ at the outer sclera ${ }^{72}$. The length of the optic nerve included in the model is $1.1 \mathrm{~cm}$. Finally, a tumor of medium size (with a maximum tumor height of $5 \mathrm{~mm}$ from the inner sclera in the $z$ direction) is modelled against the inner sclera, along the nasal side of the eye. The tumor is modelled as a segment of a sphere with a base diameter of $12 \mathrm{~mm}$ in the $y$ direction.

Scleral and corneal tissue compositions are taken from Meek ${ }^{75}$, and their density was taken to be that of 'muscle skeletal 2' from Woodard and White ${ }^{76}$ since the actual densities were not available in literature. The composition and density for the iris are also from the 'muscle skeletal 2' medium in Woodard and White ${ }^{76}$ as the iris is mostly composed of muscle ${ }^{77}$. The optic nerve, once again lacking literature data, was taken to be 'brain white matter' from Woodard and White ${ }^{76}$. Vitreous density and compositions are taken from Tumram ${ }^{78}$, aqueous from Berman ${ }^{79}$, and lens from the ICRU ${ }^{80}$. The tumor was assigned the density and composition of 'melanoma 1' from Maughan et $\mathrm{al}^{81}$. The compositions and densities used for the eye phantom are listed in Table 2.6. 


\begin{tabular}{|c|c|c|}
\hline 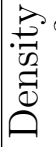 & $\frac{{ }^{\infty}}{0}$ & 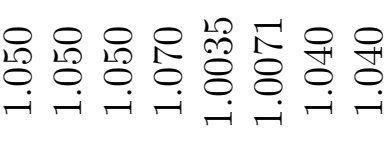 \\
\hline \multirow{9}{*}{ 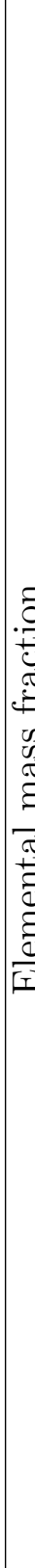 } & 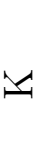 & 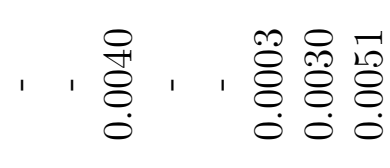 \\
\hline & $\vec{v}$ & 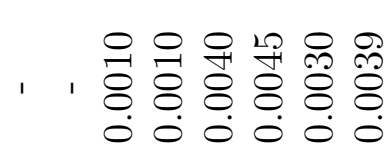 \\
\hline & ש & 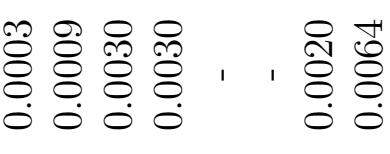 \\
\hline & $n_{1}$ & 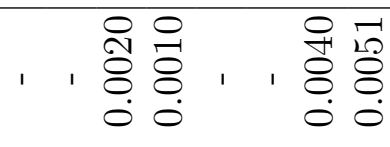 \\
\hline & $\check{z}^{\infty}$ & 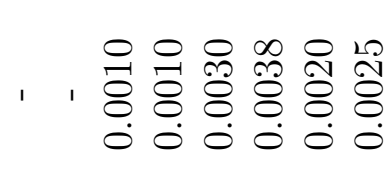 \\
\hline & 0 & 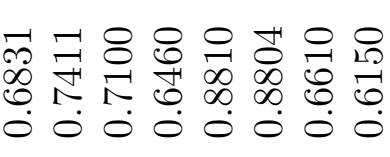 \\
\hline & 乙 & 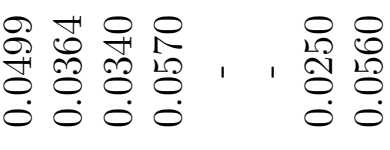 \\
\hline & U & 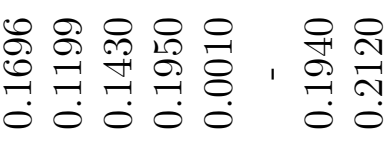 \\
\hline & $I$ & 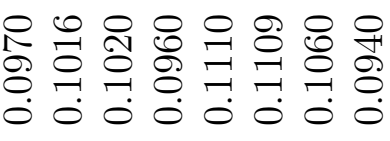 \\
\hline$\sum_{\substack{19 \\
1}}^{0}$ & 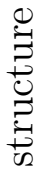 & 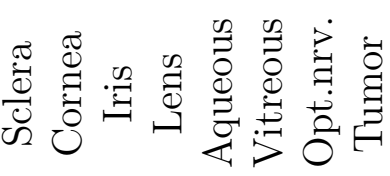 \\
\hline
\end{tabular}




\subsection{Dose calculations}

For the photon plaque simulations the default tracklength estimator scoring method ${ }^{82}$ (explained below) of egs_brachy is used. As discussed in Section 1.4.1 this method first assumes that for the purposes of low energy photons any charged particles generated will have a very short range compared to the length scales of interest. The short range of the charged particles allows the assumption of local energy deposition. Taking that assumption into account charged particle equilibrium can also be assumed. This allows for absorbed dose to be approximated as collision kerma. The tracklength dose equation differs from the generalized collision kerma equation discussed previously and is given as,

$$
D^{j}=K_{c o l}^{j}=\frac{\sum_{i} E_{i} t_{i}\left(\frac{\mu_{e n}}{\rho}\right)_{i}}{V_{j}}
$$

where $D^{j}$ is the dose to voxel $j, K_{\text {col }}^{j}$ is the collision kerma to the same voxel; $E_{i}$

is the energy of photon $i ;\left(\frac{\mu_{e n}}{\rho}\right)_{i}$ is the mass-energy absorption coefficient for that energy; $t_{i}$ is the length of the $i^{t h}$ photon's path through the voxel; and $V_{j}$ is the volume of the voxel ${ }^{83}$.

For beta plaques the main particles of interest are electrons, collision kerma is quantity for non-ionizing particles, so for ionizing particles like electrons the equality of dose and collision kerma cannot be made and absorbed dose must be calculated. In egs_brachy a high enough electron cutoff energy is needed to allow for electron transport and therefore interaction scoring.

For both photon and beta dose calculations, statistical uncertainties are calcu- 
lated using history-by-history (where a history is one of the initial, statistically independent particles) statistics ${ }^{83,84}$ to avoid difficulties that arise in uncertainty calculation for batch submitted simulations. The formula for history-by-history statistics is $^{83,84}$,

$$
S_{\bar{D}}=\sqrt{\frac{1}{N-1}\left(\frac{\sum_{i=1}^{N} D_{i}^{2}}{N}-\left(\frac{\sum_{i=1}^{N} D_{i}}{N}\right)^{2}\right)}
$$

where $S_{\bar{D}}$ is the uncertainty for the mean dose $D$ over all batches; $N$ is the number of histories; and $D_{i}$ is the quantity of dose in the $i^{\text {th }}$ batch. All simulations in this work were performed using $10^{10}$ histories.

Near to the plaque there can be voxels that contain both the medium of the plaque and the medium of the scoring region, which could result in improper scoring in that region. This can be dealt with using a volume correction. In egs_brachy the volume correction is a simple Monte Carlo calculation that determines how much of the volume of a voxel with media intersection is actually phantom. This volume information is used to correct the volume where dose will be scored. The main input for the volume correction is the density of points, which gives the number of points per cubic centimeter which will be checked during the $\mathrm{MC}$ calculation. Volume corrections for simulations in this work use a density of points equal to $10^{8}$ per $\mathrm{cm}^{3}$.

\subsubsection{Simulation parameters}

Several parameters are needed for egs_brachy to calculate dose. By default, egs_brachy utilizes a dataset file (brachy_xcom_1.5MeV.muendat) of mass-energy absorption coefficients for various media at different energies, as well as a file that contains material compositions and densities (material.dat). The photon cross sections used to generate the mass-energy absorption coefficients for egs_brachy are from the XCOM 
photon cross section database ${ }^{85}$. Radiation transport cutoffs for photon plaques and beta plaques are given in Table 2.7 .

Table 2.7: Transport parameters for photon and beta plaque simulations, where Global ECUT determines an energy below which electrons are not simulated and Global PCUT does the same for photons. Source ECUT determines an energy below which electrons are not generted by the source, but does not stop secondary particles from being simulated below that energy, and Source PCUT is the same for photons.

\begin{tabular}{|c|c|c|c|c|}
\hline $\begin{array}{c}\text { Source } \\
\text { Type }\end{array}$ & Global ECUT & Global PCUT & Source ECUT & Source PCUT \\
\hline Photon & $1.512 \mathrm{MeV}$ & $0.001 \mathrm{MeV}$ & $1.512 \mathrm{MeV}$ & $0.001 \mathrm{MeV}$ \\
\hline Beta & $0.522 \mathrm{MeV}$ & $0.010 \mathrm{MeV}$ & $0.522 \mathrm{MeV}$ & $0.010 \mathrm{MeV}$ \\
\hline \hline
\end{tabular}

\subsubsection{Dose normalization}

egs_brachy outputs dose in the form of dose per history, where the number of histories is the number of initial, statistically independent particles. To normalize this output to absolute dose several parameters must be taken into account. For a low dose rate photon source like those used in this work the formula for egs_brachy to convert from dose per history to absolute dose is as follows ${ }^{83}$,

$$
D_{t o t}^{j}=\left[S_{k}\right]_{\text {max }} \frac{\bar{D}^{j}}{S_{K}^{h i s t}} \tau\left(1-e^{-\lambda t_{t o t}}\right)
$$

where $D_{t o t}^{j}$ is the absolute dose to voxel $j ;\left[S_{k}\right]_{\max }$ is the initial maximum air-kerma strength of the chosen brachytherapy sources; $\bar{D}^{j}$ is the dose per history output by egs_brachy; $S_{K}^{\text {hist }}$ is the air-kerma strength per history for the sources; $\tau$ is the mean lifetime of the sources; $\lambda$ is the decay constant of the sources; and $t_{t o t}$ is the total treatment time for the procedure. The values used for the ${ }^{125} \mathrm{I}$ and ${ }^{103} \mathrm{Pd}$ sources in this work can be found in Table 2.8 . 
Table 2.8: Parameters used for calculation of absolute dose with ${ }^{125} \mathrm{I}$ and ${ }^{103} \mathrm{Pd}$ seeds. The unit of air kerma strength $U$ is given by $\left[\frac{\mathrm{CGy} \mathrm{cm}{ }^{2}}{\mathrm{~h}}\right]$.

\begin{tabular}{|c|c|c|c|c|c|}
\hline Source & {$\left[S_{k}\right]_{\max }(U)$} & $S_{K}^{\text {hist }}\left(E^{-14} \frac{U}{\text { hist }}\right)$ & $\tau(d)$ & $\lambda\left(d^{-1}\right)$ & $t_{\text {tot }}(h)$ \\
\hline${ }^{125} \mathrm{I}$ & 4.572 & 3.769 & 41.17 & 0.01167 & 100 \\
\hline${ }^{103} \mathrm{Pd}$ & 3.879 & 6.428 & 11.78 & 0.0408 & 168 \\
\hline \hline
\end{tabular}

However, not all results in literature are presented in terms of absolute dose and so a different normalization may be used. Many of the photon plaque calculations in this work are scaled such that $D_{T G 43}$ is 85 Gy at the prescription dose point of the tumor apex $(0,0,0.5) \mathrm{cm}$. This was done to compare to the MCNP5 calculations of Melhus and Rivard ${ }^{24}$.

For beta plaques, when comparing to most of the previous calculations (eg. those using PENELOPE $\left.{ }^{86}\right)$ normalization is done to $100 \%$ at $(0,0,0.1) \mathrm{cm}$. For comparisons to the calculations from Sommer's GEANT4 paper ${ }^{49}$ the dose is instead normalized to Gy per decay. This is done by dividing dose per history by the particle fluence per history.

When comparing the photon and beta plaque results to each other $D_{w, w}$ is scaled to $100 \%$ at the tumor apex $((0.25,0.25,-0.675) \mathrm{cm}$ in eye centre coordinates $), D_{m, m}$ is then scaled using the same value.

\subsection{Calculation specifications}

The dose calculations in this work include three possible model configurations. First, simulations are done for photon plaques which are meant to emulate TG43 (referred to as $D_{T G 43}$ ). $D_{T G 43}$ calculations are done with seeds in a water phantom (see Table 2.5) and no plaque model is included. The dose is calculated for each seed, one at a time and then summed through superposition to avoid interseed effects (attenuation of photons emitted by one seed caused by another seed). egs_brachy 
has a superposition run mode that makes this a simple task. Second, dose calculations which take into account the plaque geometry and involve radiation transported and scored in water are represented by $D_{w, w}{ }^{87}$. For photon plaques these simulations calculate dose with seeds inserted into a model of the plaque as described in Section 2.1.1. The plaque is in turn inserted into a water phantom. For beta plaques these simulations calculate dose with the full silver plaque model and source film in a similar water phantom (see Table 2.5). Both $D_{T G 43}$ and $D_{w, w}$ calculations set the origin at the eye plaque coordinate origin of the inner sclera. Last, dose calculations using the anatomically realistic voxelized eye phantom from Lesperance et $\mathrm{al}^{52}$ where radiation travels through eye media and is scored in eye media are referred to as $D_{m, m} . D_{m, m}$ calculations are done with both photon and beta plaques as well and the origin set at the eye centre.

All calculations in this thesis were done using egs_brachy version v2017.09.15 on the Carleton physics research server cluster of 644 cores. Each simulation was run with $10^{10}$ histories. For photon plaques the calculations of $D_{T G 43}$ and $D_{w, w}$ took between 21 and $35 \mathrm{CPU}$ hours, while calculations of $D_{m, m}$ can take as many as 50 CPU hours. For beta plaques calculations take much longer due to the need for interaction scoring. $D_{w, w}$ can take up to $720 \mathrm{CPU}$ hours and $D_{m, m} 750 \mathrm{CPU}$ hours.

Dose results are extracted from along the central axis for each of these calculations. In addition to central axis doses, values for four points of clinical interest in the eye at eight different eye plaque positions are extracted. The points of interest include the fovea, optic disc, lens, and lacrimal gland which are located at $(-1.13$, $0,0) \mathrm{cm},(-1.06,0.4,0) \mathrm{cm},(0.77,0,0) \mathrm{cm}$, and $(0.77,0.82 .0 .82) \mathrm{cm}$ respectively, where the coordinates are with respect to the eye centre. The eight plaque positions are:

\#1: on the temporal side of the eye, centered on the equator 
\#2: on the nasal side of the eye, centered on the equator

\#3: on the superior side of the eye, centered on the equator

\#4: on the inferior side of the eye, centered on the equator

\#5: on the temporal side of the eye, posterior from the equator

\#6: on the nasal side of the eye, posterior from the equator

\#7: on the superior side of the eye, posterior from the equator

\#8: on the inferior side of the eye, posterior from the equator

For beta plaques, dose profiles along the lateral axis at different depths with respect to the eye plaque $(0.1 \mathrm{~cm}, 0.2 \mathrm{~cm}, 0.3 \mathrm{~cm}, 0.4 \mathrm{~cm}, 0.6 \mathrm{~cm}$, and $0.8 \mathrm{~cm})$ are extracted.

Comparisons $\triangle \mathrm{D}_{L O C A L}$ and $\triangle \mathrm{D}_{G L O B A L}{ }^{88,89}$ are used as an overall comparison metric, where $\Delta \mathrm{D}_{\mathrm{LOCAL}}=\frac{\mathrm{D}_{\mathrm{A}}(r)-\mathrm{D}_{\mathrm{B}}(r)}{\mathrm{D}_{\mathrm{B}}(r)}$ and $\Delta \mathrm{D}_{\mathrm{GLOBAL}}=\frac{\mathrm{D}_{\mathrm{A}}(r)-\mathrm{D}_{\mathrm{B}}(r)}{\mathrm{D}_{\mathrm{B}}\left(r_{r e f}\right)}$ and $r_{r e f}$ is given by dose at the reference tumor apex point $(0,0,0.5) \mathrm{cm}$ within the eye plaque coordinate system. These results are given for both photon seed types.

\subsubsection{Uncertainties}

\section{Statistical uncertainties}

For the purposes of discussing statisitical uncertainties it should be stated that the tumor apex is located at $z=0.5 \mathrm{~cm}$, and the eye centre and opposite side of the eye are at $z=1.23 \mathrm{~cm}$ and $z=2.46 \mathrm{~cm}$ respectively. The statistical uncertainties for the egs_brachy photon plaque dose calculations presented here are less than $0.1 \%$ at the tumor apex and up to $0.4 \%$ at the opposite side of the eye. For beta plaques the statistical uncertainties are less than $0.3 \%$ within the tumor and $1 \%$ up to the eye centre. Across the lateral points the largest uncertainties are $2.4 \%$, but the majority are below 1\%. The BrachyDose calculations from the CLRP eye 
plaque database have statistical uncertainties similar to those of the egs_brachy calculations, specifically $0.1 \%$ within the tumor and $0.5 \%$ up to the opposite side of the eye ${ }^{43}$. The BrachyDose dose calculations in the Rivard 2011 paper are reported with statistical uncertainties up to $0.1 \%$ within the tumor and $0.5 \%$ at the opposite side of the eye ${ }^{42}$. The MCNP5 dose calculations are reported to have statistical uncertainties up to $0.9 \%$ within the tumor and $2.0 \%$ at the opposite side of the eye $^{24,42}$. The PENELOPE dose calculation statistical uncertainties are reported as generally less than $2 \%^{47}$. The GEANT4 dose calculation statistical uncertainties are reported as $0.12 \%$ within the tumor and $0.4 \%$ up to the eye centre ${ }^{49}$.

\section{Other uncertainties}

Sources of uncertainty other than statistical uncertainties are important to consider and in fact likely account for a greater uncertainty in the calculations than statistics. The TG43U1 ${ }^{34}$ update includes an uncertainty analysis for Monte Carlo brachytherapy calculations that concludes a $2 \%$ uncertainty in both photon cross sections and seed geometries near the seed. There are uncertainties to consider in the plaque models as well.

The suture eyelets on the plaques have not been modelled, though they are small and out of the way and so unlikely to have a large effect on dose. For photon plaques the seeds are modelled to be completely enveloped in the insert material. In reality the seeds are slotted into the insert, so small air pockets would exist. The radionuclide coated film that is the source for beta plaques is not geometrically modelled. The beta plaques are modelled as a single piece. The film is on the order of $0.1 \mu \mathrm{m}$ thick and its exclusion is unlikely to have large effects. It is also assumed that the beta plaque sources have a uniform activity distribution, which BEBIG certificates show may not be the case. 


\section{Chapter 3}

\section{Results}

In this chapter, doses calculated in egs_brachy are presented for both the photon and beta plaques. These doses are shown alongside MC calculated doses from previous studies using MCNP $5^{24,42}$, BrachyDose ${ }^{42,43}$, PENELOPE $^{47}$ and GEANT4 ${ }^{49}$. For

photon plaques most dose data are presented using both ${ }^{125} \mathrm{I}$ and ${ }^{103} \mathrm{Pd}$ seeds and include calculations of $D_{T G 43}, D_{w, w}$, and $D_{m, m}$. For beta plaques dose calculations are made with the source nuclide ${ }^{106} \mathrm{Ru}$ and both $D_{w, w}$, and $D_{m, m}$ are presented. Various graphs and tables comparing egs_brachy photon and beta plaque results conclude the chapter. In some places, two doses $\left(D_{a}, D_{b}\right)$ are compared via their relative difference, defined as $\left|1-D_{a} / D_{b}\right| \times 100 \%$ (e.g. $D_{a}=76$ Gy and $D_{b}=85$ Gy, $|1-76 / 85| \times 100 \%=10.6 \%)$.

\subsection{Photon plaque results}

$D_{T G 43}$ depth-dose curves along the plaque central axis are given for COMS $10 \mathrm{~mm}$ to COMS $22 \mathrm{~mm}$ (and with both $6711{ }^{125} \mathrm{I}$ seeds and T200 ${ }^{103} \mathrm{Pd}$ seeds) in the subfigures of Figures 3.1 and 3.2. The doses in the majority of these figures are scaled to 85 Gy at the reference tumor apex $((0,0,0.5) \mathrm{cm}$ in the eye plaque coordinate system) to match the common prescription dose used with the TG43 protocol. The doses in the subfigures (a) of Figures 3.1 and 3.2 are calculated to be absolute doses 
and are done with a COMS $16 \mathrm{~mm}$ plaque (see Section 2.3 for the parameters used to determine absolute dose for the two different seed types). The depth range of the prescription dose scaled figures is limited by the amount of data presented for MCNP5 ${ }^{24}$ and only reach the eye centre. The absolute dose calculations in Rivard et al. have points at further depths and therefore allow for a more complete depth-dose curve from the inner sclera to the opposite sclera ${ }^{42}$.

For $D_{T G 43}$ (Figures 3.1 and 3.2), when using ${ }^{125} \mathrm{I}$ seeds, the relative difference between egs_brachy and MCNP5 at depths up to the centre of the eye is less than $0.8 \%$ for the best agreement (COMS $10 \mathrm{~mm}$ ) and up to $2.1 \%$ for the worst agreement (COMS $22 \mathrm{~mm}$ ). The agreement tends to get better as plaque size decreases. When using ${ }^{103} \mathrm{Pd}$ seeds the relative difference between egs_brachy and MCNP5 at depths up to the centre of the eye is less than $1.9 \%$ for the best agreement (COMS $22 \mathrm{~mm}$ ) and up to $3.3 \%$ for the worst agreement (COMS $20 \mathrm{~mm}$ ). There does not appear to be a trend between plaque size and agreement when using the ${ }^{103} \mathrm{Pd}$ seeds. The largest statistical uncertainties for the egs_brachy dose results are $0.14 \%$ for ${ }^{125} \mathrm{I}$ and $0.17 \%$ for ${ }^{103} \mathrm{Pd}$.

For the absolute $D_{T G 43}$ depth-dose curves (subfigure (a) of both Figures 3.1 and 3.2) the relative differences between egs_brachy and BrachyDose calculated doses using ${ }^{125} \mathrm{I}$ are less than $0.5 \%$ up to the tumor apex and $0.7 \%$ up to the opposite side of the eye. Between egs_brachy and MCNP5, 1.4\% is the largest relative difference for both tumor apex and opposite side of the eye. When comparing absolute doses calculated with ${ }^{103} \mathrm{Pd}$, egs_brachy and BrachyDose disagree by only $0.5 \%$ up to the tumor apex and $0.6 \%$ up to the opposite side of the eye. egs_brachy and MCNP5 disagree by $2.2 \%$ and $3.4 \%$ for up to tumor apex and the opposite side of the eye respectively. 
The absolute doses for $D_{T G 43}$ (at the points of interest discussed in Section 2.4) given in Table 3.1 and show a relative difference between egs_brachy and BrachyDose of less than $1 \%$ for all points and for both seed types. For ${ }^{125} \mathrm{I}$ the relative difference between egs_brachy and MCNP5 are generally within $2 \%$ and there are only a few outliers. For ${ }^{103} \mathrm{Pd}$ these outliers have a relative difference of between $2 \%$ and $9 \%$. 

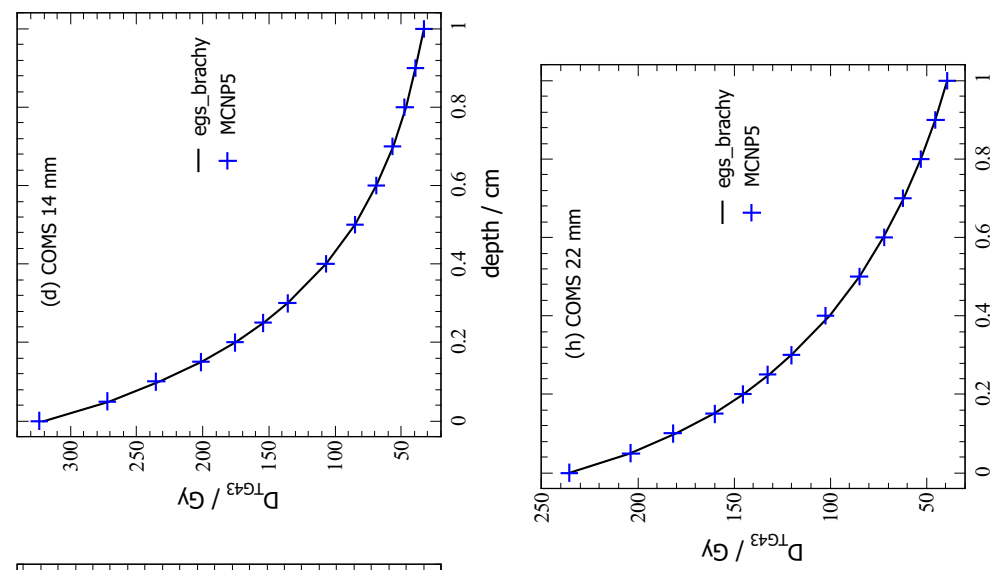

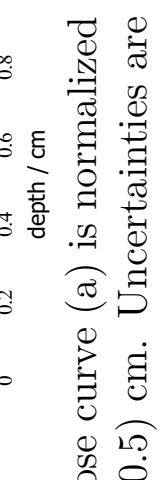
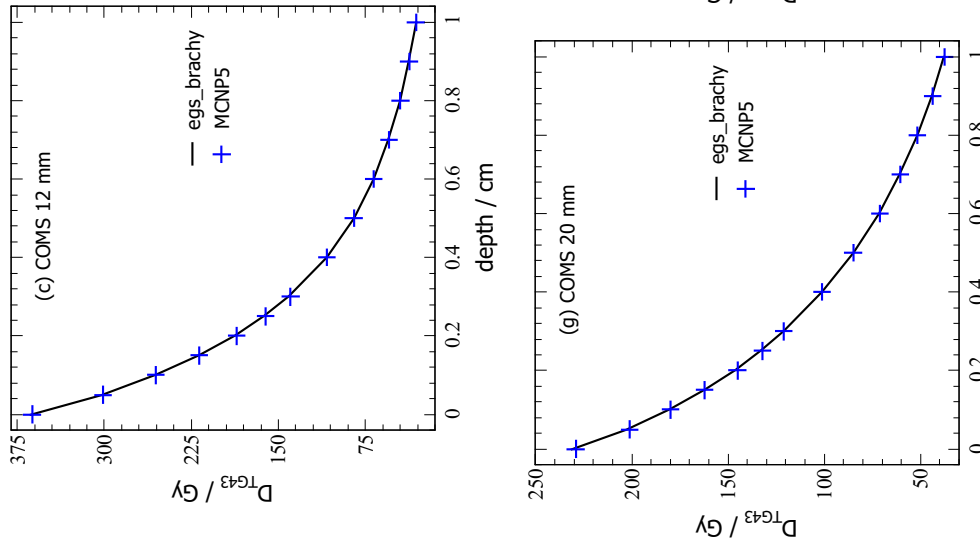

ค
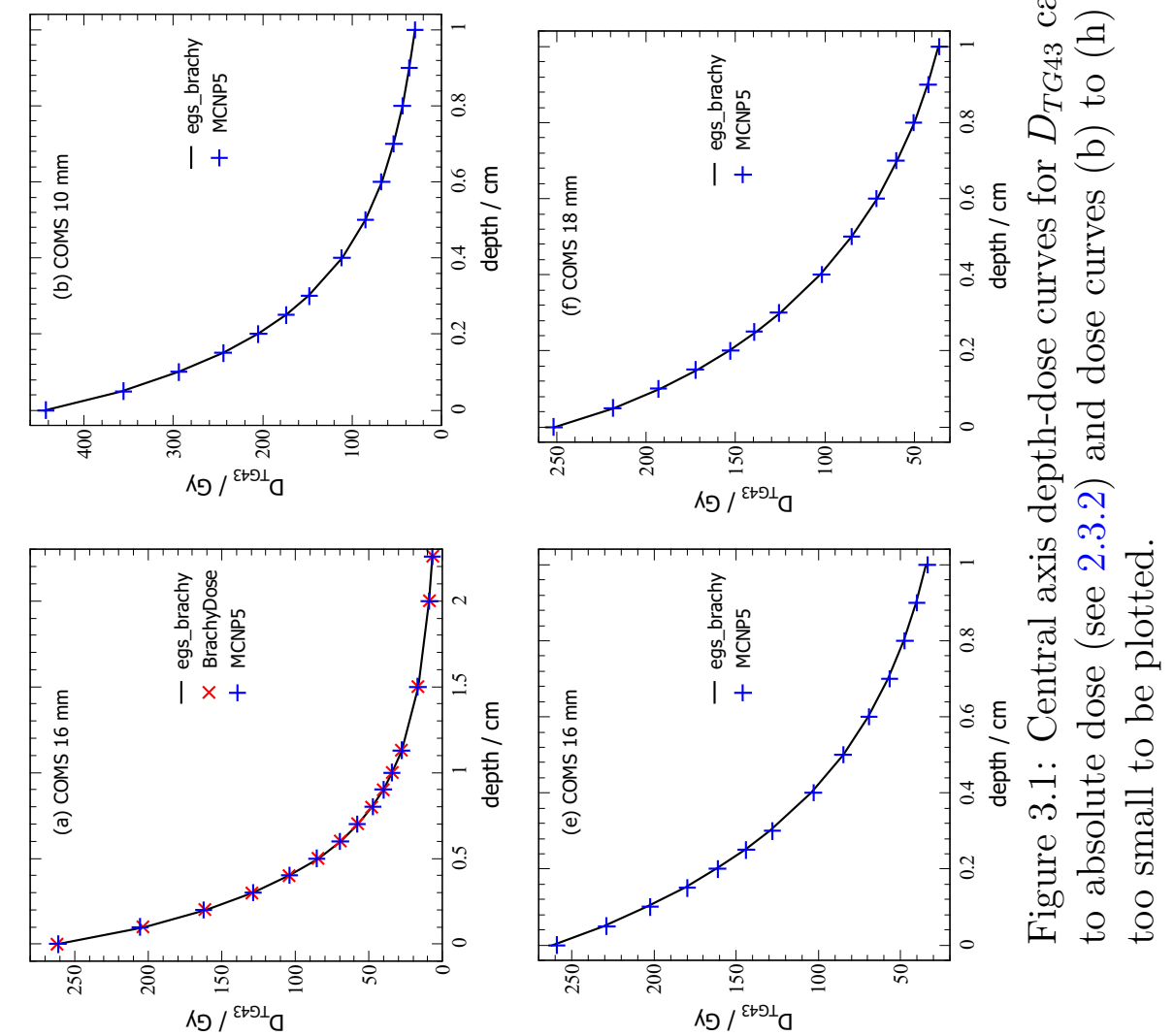

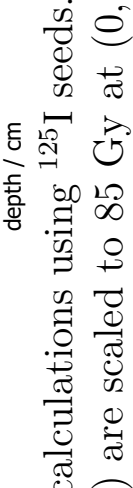
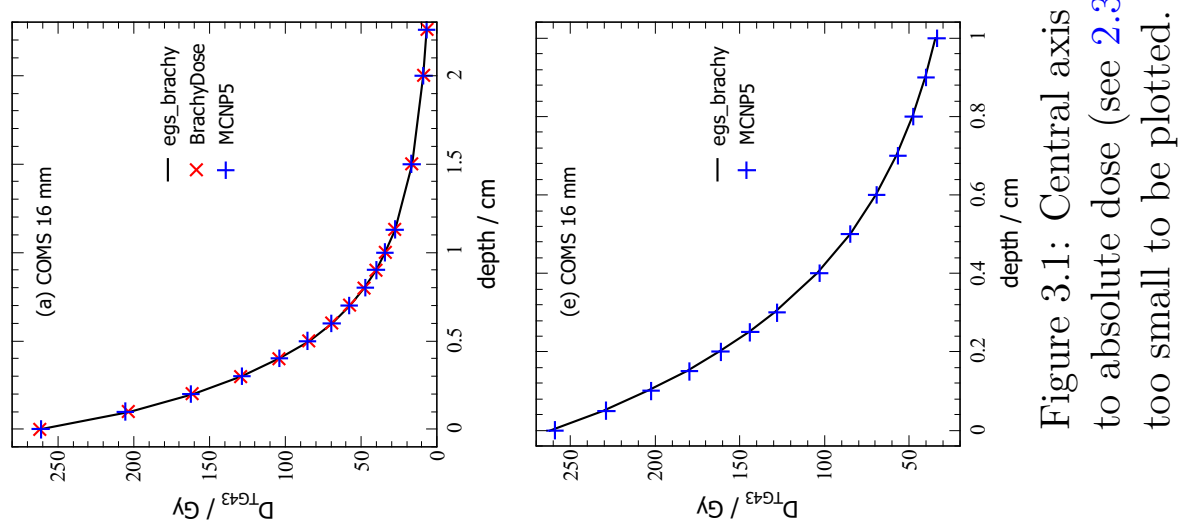

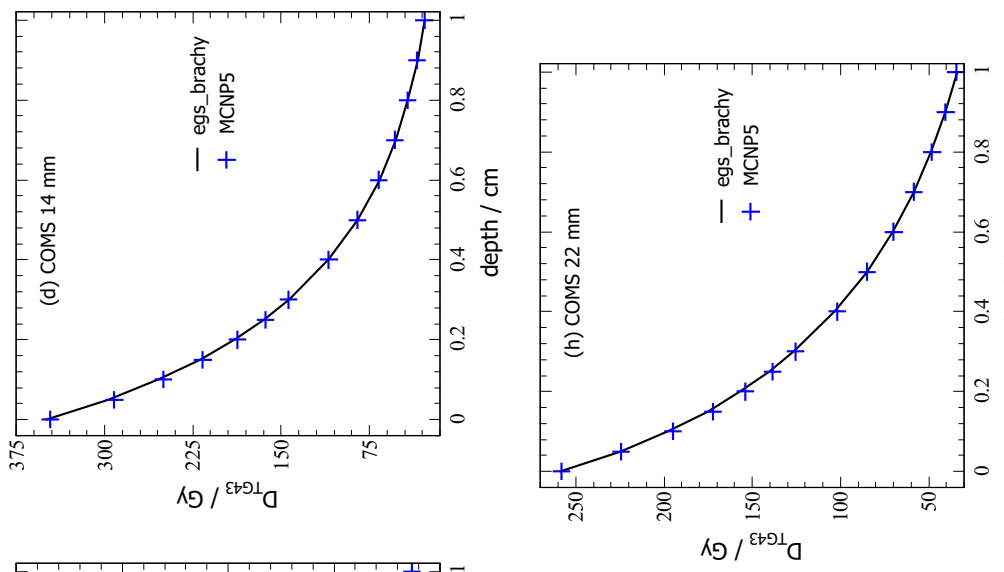

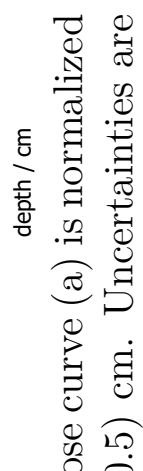
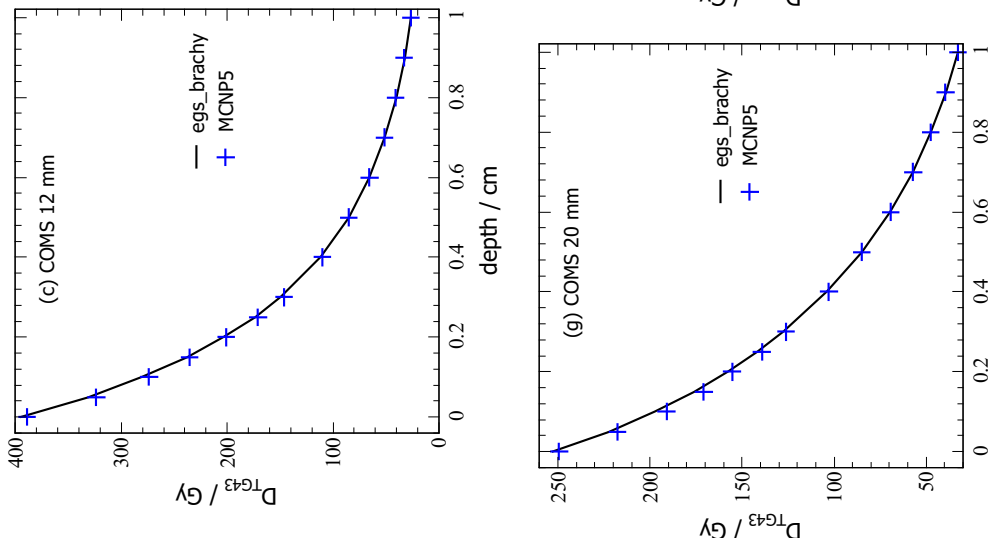

$\stackrel{\circ}{\circ}$
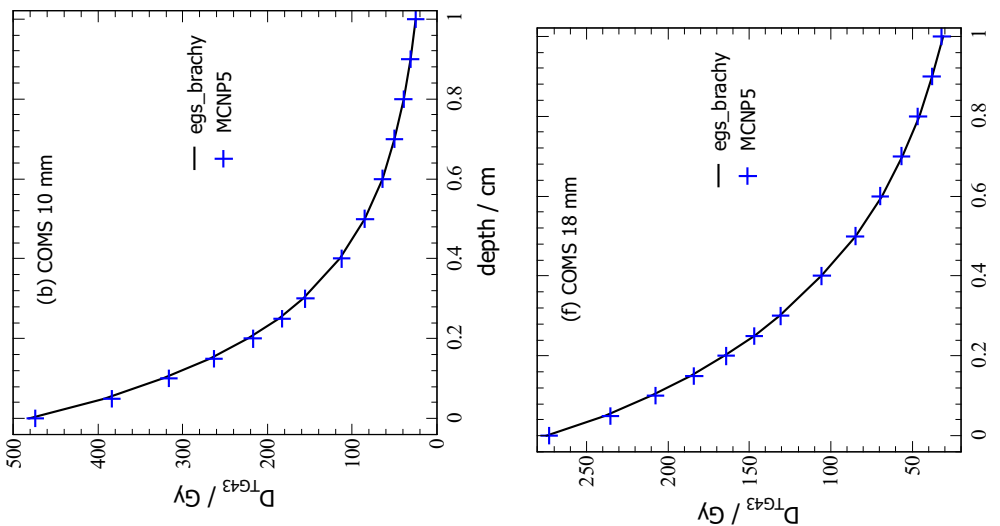

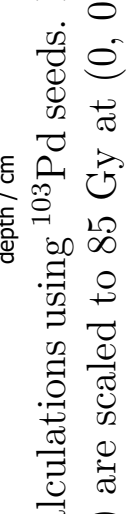
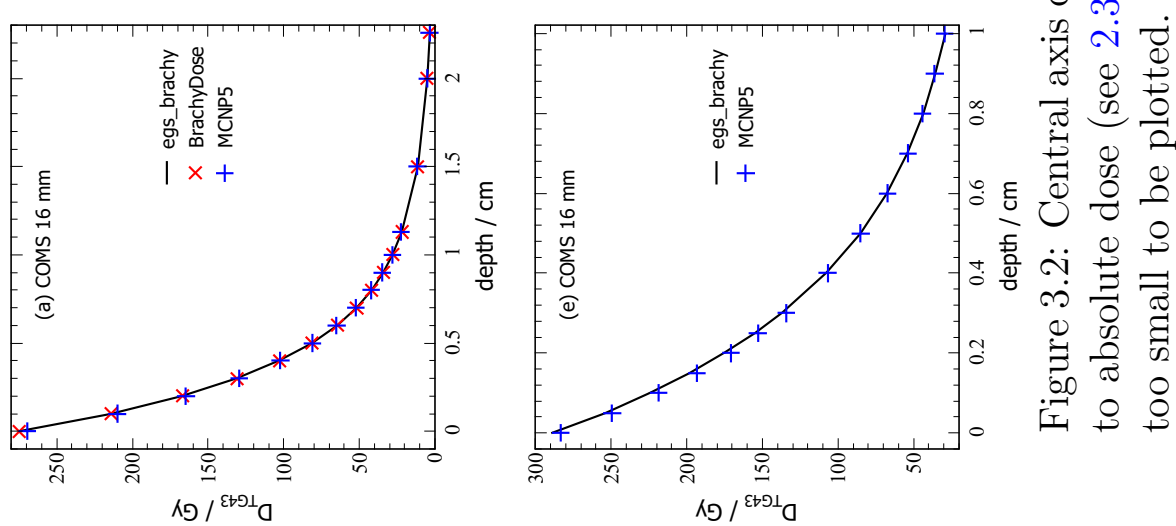
Table 3.1: $D_{T G 43}$ doses for egs_brachy at different plaque locations and points of interest. Ratios are given between egs_brachy and both BrachyDose and MCNP5. Both ${ }^{125} \mathrm{I}$ and ${ }^{103} \mathrm{Pd}$ results are shown.

\begin{tabular}{|c|c|c|c|c|c|c|c|}
\hline \multirow[b]{2}{*}{$\begin{array}{l}\text { plq. } \\
\text { pos. }\end{array}$} & \multirow[b]{2}{*}{$\begin{array}{c}\text { point of } \\
\text { interest }\end{array}$} & \multicolumn{3}{|c|}{${ }^{125} I$} & \multicolumn{3}{|c|}{${ }^{103} \mathrm{Pd}$} \\
\hline & & $\begin{array}{l}D_{\mathrm{eb}} \\
(\mathrm{Gy})\end{array}$ & $\left(\frac{D_{e b}}{D_{B D}}\right)$ & $\left(\frac{D_{\mathrm{eb}}}{\mathrm{D}_{\mathrm{MCNP}}}\right)$ & $\begin{array}{c}\mathrm{D}_{\mathrm{eb}} \\
(\mathrm{Gy})\end{array}$ & $\left(\frac{D_{\mathrm{eb}}}{\mathrm{D}_{\mathrm{BD}}}\right)$ & $\left(\frac{D_{\mathrm{eb}}}{\mathrm{D}_{\mathrm{MCNP} 5}}\right)$ \\
\hline \multirow{4}{*}{$\# 1$} & fovea & 16.4 & 1.00 & 1.01 & 10.9 & 1.00 & 1.04 \\
\hline & opt. dsc. & 11.3 & 1.00 & 0.99 & 6.8 & 1.00 & 1.02 \\
\hline & lens & 21.7 & 1.00 & 1.01 & 15.6 & 1.00 & 1.06 \\
\hline & lac. gld. & 39.8 & 1.00 & 1.03 & 30.7 & 1.00 & 1.04 \\
\hline \multirow{4}{*}{$\# 2$} & fovea & 16.4 & 1.00 & 1.01 & 10.9 & 1.00 & 1.04 \\
\hline & opt. dsc. & 27.6 & 1.00 & 1.01 & 20.7 & 1.00 & 1.04 \\
\hline & lens & 21.7 & 1.00 & 1.01 & 15.6 & 1.00 & 1.06 \\
\hline & lac. gld. & 7.2 & 1.00 & 1.04 & 3.9 & 1.01 & 1.04 \\
\hline \multirow{4}{*}{$\# 3$} & fovea & 16.4 & 1.00 & 1.01 & 10.9 & 1.00 & 1.04 \\
\hline & opt. dsc. & 16.5 & 1.00 & 1.02 & 10.9 & 1.00 & 1.05 \\
\hline & lens & 21.7 & 1.00 & 1.01 & 15.6 & 1.00 & 1.06 \\
\hline & lac. gld. & 39.8 & 1.00 & 1.03 & 30.8 & 1.00 & 1.04 \\
\hline \multirow{4}{*}{$\# 4$} & fovea & 16.4 & 1.00 & 1.01 & 10.9 & 1.00 & 1.04 \\
\hline & opt. dsc. & 16.4 & 1.00 & 1.02 & 10.9 & 1.00 & 1.04 \\
\hline & lens & 21.7 & 1.00 & 1.01 & 15.6 & 1.00 & 1.06 \\
\hline & lac. gld. & 7.3 & 1.01 & 1.04 & 3.9 & 1.00 & 1.03 \\
\hline \multirow{4}{*}{$\# 5$} & fovea & 73.6 & 0.99 & 1.01 & 66.5 & 0.99 & 1.09 \\
\hline & opt. dsc. & 31.0 & 1.00 & 1.01 & 23.9 & 1.00 & 1.06 \\
\hline & lens & 12.3 & 1.00 & 0.99 & 7.7 & 1.00 & 1.04 \\
\hline & lac. gld. & 13.5 & 1.00 & 1.03 & 8.4 & 1.00 & 1.03 \\
\hline \multirow{4}{*}{$\# 6$} & fovea & 27.1 & 1.00 & 1.00 & 20.2 & 1.00 & 1.04 \\
\hline & opt. dsc. & 59.2 & 1.00 & 1.01 & 51.5 & 1.00 & 1.07 \\
\hline & lens & 15.9 & 0.99 & 0.99 & 10.7 & 1.00 & 1.02 \\
\hline & lac. gld. & 6.4 & 1.00 & 1.02 & 3.3 & 1.01 & 1.12 \\
\hline \multirow{4}{*}{$\# 7$} & fovea & 73.6 & 0.99 & 1.01 & 66.5 & 0.99 & 1.09 \\
\hline & opt. dsc. & 60.2 & 1.00 & 1.00 & 51.1 & 1.00 & 1.02 \\
\hline & lens & 12.3 & 1.00 & 0.99 & 7.7 & 1.00 & 1.04 \\
\hline & lac. gld. & 13.5 & 1.00 & 1.01 & 8.4 & 0.99 & 1.06 \\
\hline \multirow{4}{*}{$\# 8$} & fovea & 73.6 & 0.99 & 1.01 & 66.5 & 0.99 & 1.09 \\
\hline & opt. dsc. & 60.3 & 1.00 & 0.98 & 51.1 & 1.00 & 1.02 \\
\hline & lens & 12.3 & 1.00 & 0.99 & 7.7 & 1.00 & 1.04 \\
\hline & lac. gld. & 6.1 & 1.00 & 1.02 & 3.0 & 0.99 & 1.04 \\
\hline
\end{tabular}


The depth-dose curves for simulations of $D_{w, w}$ using both ${ }^{125} \mathrm{I}$ and ${ }^{103} \mathrm{Pd}$ are given in Figures 3.3 and 3.4. The majority of these central axis sub-figures are scaled using the value needed for $D_{T G 43}$ to be 85 Gy at the prescription point $(0,0,0.5)$ cm; however, once again, sub-figures (a) of Figures 3.3 and 3.4 are absolute dose calculations (see Section 2.3 for the parameters used to determine absolute dose for the two seed types). The egs_brachy doses and ratios to other MC codes are given in Table 3.2 for the different points of interest discussed in Section 2.4.

For $D_{w, w}$ using ${ }^{125} \mathrm{I}$ seeds, the largest disagreement between egs_brachy and BrachyDose at depths up to the tumor apex is less than $0.3 \%$ to $0.6 \%$ for all plaque models, and up to the eye centre is also less than $0.3 \%$ to $0.6 \%$ for all plaque models. The best agreement is with the COMS $12 \mathrm{~mm}$ plaque and the worst with COMS $22 \mathrm{~mm}$ plaque, but there does not appear to be a trend relating to plaque size. When using ${ }^{103} \mathrm{Pd}$ seeds the relative difference between egs_brachy and BrachyDose at depths up to the tumor apex ranges from less than $0.3 \%$ to $0.8 \%$ for all plaque models, and at depths up to the eye centre is less than between $0.4 \%$ and $1.0 \%$ across all plaques. In this case, the best agreement is with the COMS $10 \mathrm{~mm}$ plaque and the worst is the COMS $20 \mathrm{~mm}$ plaque. It should be noted that very few central axis doses have greater than $0.001 \%$ relative difference between egs_brachy and BrachyDose when using ${ }^{103} \mathrm{Pd}$ seeds.

When comparing $D_{w, w}$ with MCNP5 using ${ }^{125}$ I seeds, relative differences are at most $0.5 \%$ to $1.8 \%$ up to the tumor apex and $0.7 \%$ to $2.4 \%$ up to the eye centre across all plaque models. The best agreement exists with the COMS $10 \mathrm{~mm}$ and the worst with the COMS $22 \mathrm{~mm}$. Here the differences do appear to increase with the plaque size. With ${ }^{103} \mathrm{Pd}$ seeds dose relative differences range between $0.9 \%$ and $1.9 \%$ up to the tumor apex and between $2.0 \%$ and $3.4 \%$ up to the eye centre, once again for all plaque models. The worst agreement is with the COMS $14 \mathrm{~mm}$ and the best 
the COMS $16 \mathrm{~mm}$.

For the absolute $D_{w, w}$ depth-dose curves (subfigure (a) of both Figures 3.3 and 3.4) the relative differences between doses calculated with egs_brachy and BrachyDose using ${ }^{125} \mathrm{I}$ are less $0.3 \%$ up to the tumor apex and $0.9 \%$ up to the opposite side of the eye. Between egs_brachy and MCNP5 the largest relative difference is $2.4 \%$ for both tumor apex and opposite side of the eye. When comparing absolute doses calculated with ${ }^{103} \mathrm{Pd}$, egs_brachy and BrachyDose disagree by $0.5 \%$ up to the tumor apex and $1.4 \%$ up to the opposite side of the eye. egs_brachy and MCNP5 disagree by $2.2 \%$ and $3.4 \%$ up to tumor apex and up to the opposite side of the eye respectively.

For both $D_{T G 43}$ and $D_{w, w}$ agreement between egs_brachy and MCNP5 is comparable to agreement between BrachyDose and MCNP5. In some cases the agreement seems to be related to plaque size, but not often enough to be considered a strong trend. Agreement also tends to be stronger with ${ }^{125} \mathrm{I}$ seeds over ${ }^{103} \mathrm{Pd}$ seeds. Larger disagreements are commonly at dose locations that are at a further depths and/or are in the penumbra region of the plaque. This is shown in the dose ratios at points of interest in Table 3.2 (e.g. the lacrimal gland for positions \#1 and \#3 of the plaque). 

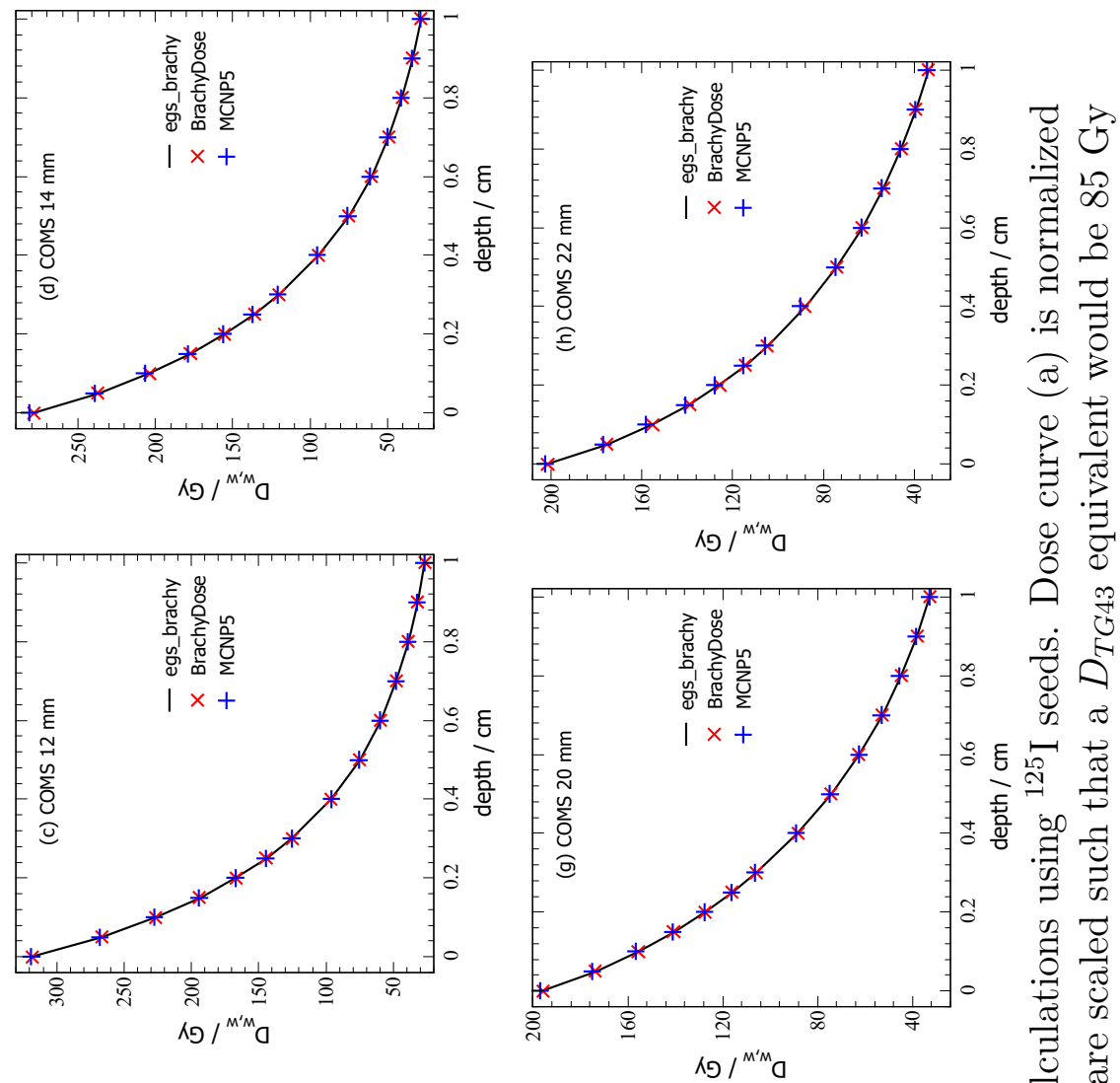

(2)
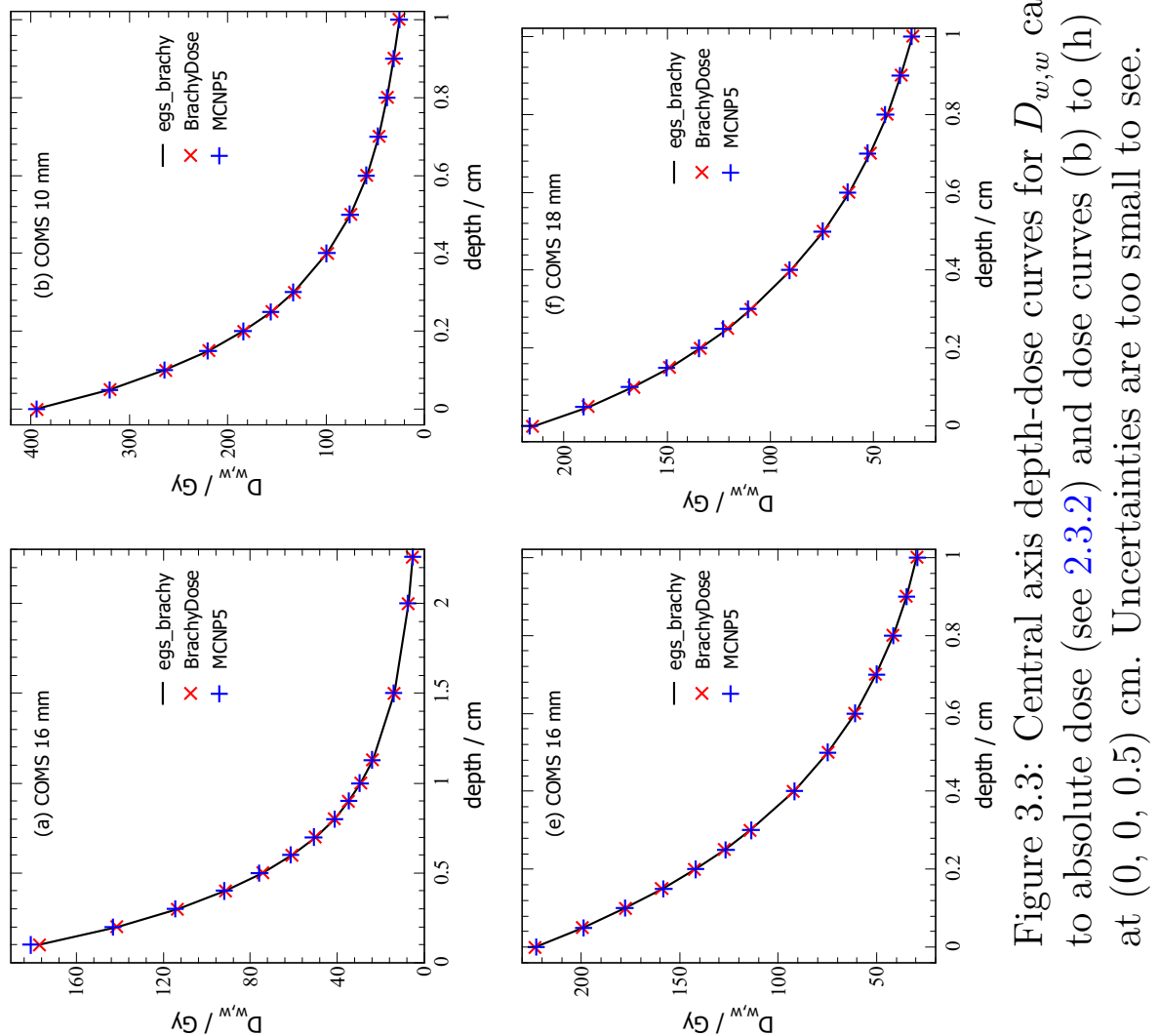

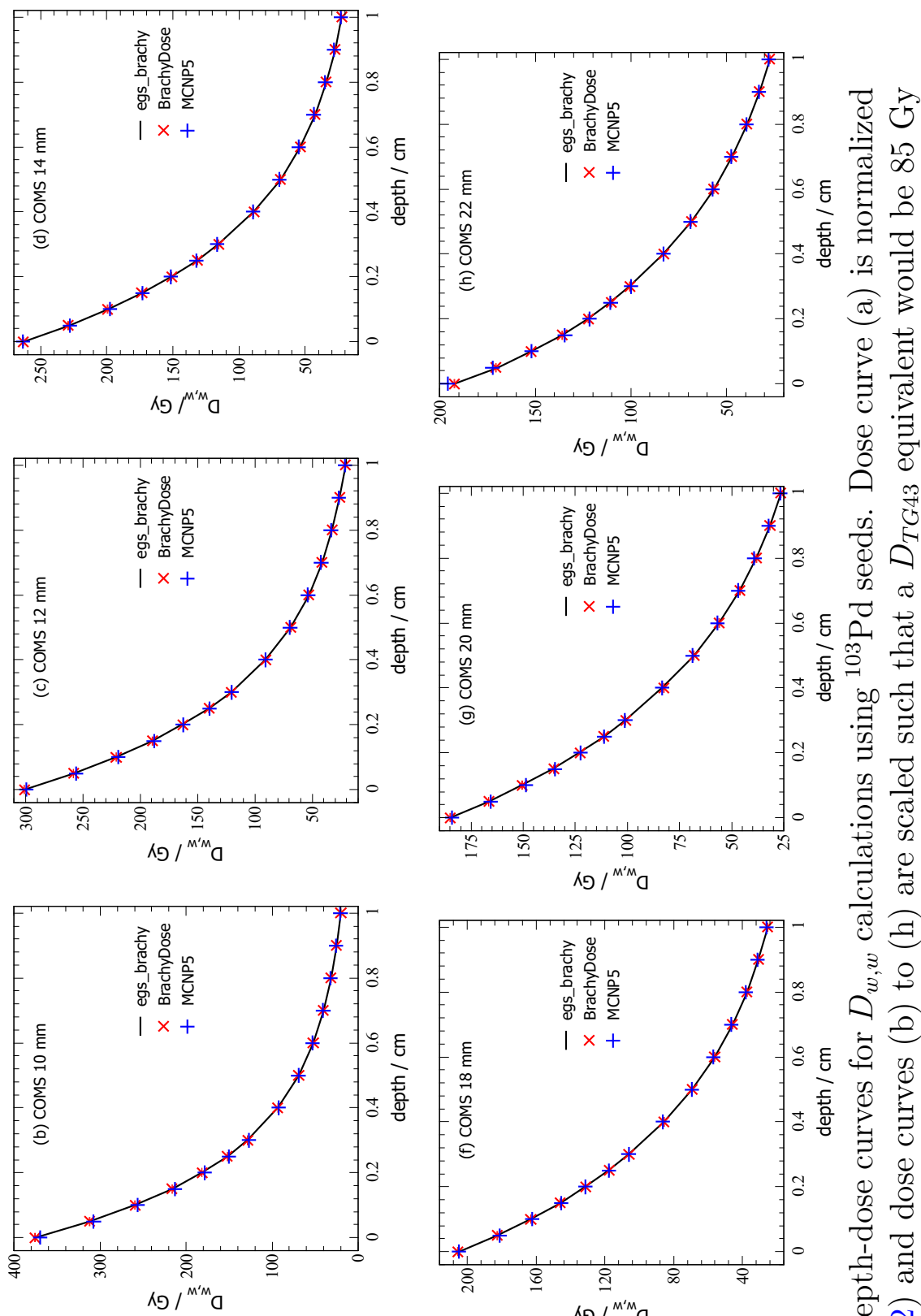

คำ 
Table 3.2: $D_{w, w}$ doses for egs_brachy at different plaque locations and points of interest. Ratios are given between egs_brachy and both BrachyDose and MCNP5. Both ${ }^{125} \mathrm{I}$ and ${ }^{103} \mathrm{Pd}$ results are shown.

\begin{tabular}{|c|c|c|c|c|c|c|c|}
\hline \multirow[b]{2}{*}{$\begin{array}{l}\text { plq. } \\
\text { pos. }\end{array}$} & \multirow[b]{2}{*}{$\begin{array}{l}\text { point of } \\
\text { interest }\end{array}$} & \multicolumn{3}{|c|}{${ }^{125} I$} & \multicolumn{3}{|c|}{${ }^{103} \mathrm{Pd}$} \\
\hline & & $\begin{array}{l}\mathrm{D}_{\mathrm{eb}} \\
(\mathrm{Gy})\end{array}$ & $\left(\frac{D_{e b}}{D_{B D}}\right)$ & $\left(\frac{D_{\mathrm{eb}}}{\mathrm{D}_{\mathrm{MCNP}}}\right)$ & $\begin{array}{c}\mathrm{D}_{\mathrm{eb}} \\
(\mathrm{Gy})\end{array}$ & $\left(\frac{D_{e b}}{D_{B D}}\right)$ & $\left(\frac{D_{\text {eb }}}{D_{\text {MCNP5 }}}\right)$ \\
\hline \multirow{4}{*}{$\# 1$} & fovea & 12.7 & 0.99 & 0.98 & 7.7 & 1.00 & 1.03 \\
\hline & opt. dsc. & 9.0 & 1.00 & 0.99 & 5.0 & 0.99 & 0.99 \\
\hline & lens & 17.9 & 1.00 & 0.99 & 12.0 & 1.01 & 1.06 \\
\hline & lac. gld. & 6.6 & 0.98 & 1.21 & 3.7 & 0.98 & 1.27 \\
\hline \multirow{4}{*}{$\# 2$} & fovea & 12.7 & 0.99 & 0.98 & 7.7 & 1.00 & 1.03 \\
\hline & opt. dsc. & 20.7 & 1.00 & 0.97 & 13.5 & 1.00 & 1.02 \\
\hline & lens & 17.9 & 1.00 & 0.99 & 12.0 & 1.01 & 1.06 \\
\hline & lac. gld. & 5.6 & 0.99 & 0.99 & 2.9 & 1.01 & 1.04 \\
\hline \multirow{4}{*}{$\# 3$} & fovea & 12.7 & 0.99 & 0.98 & 7.7 & 1.00 & 1.03 \\
\hline & opt. dsc. & 12.8 & 1.00 & 1.00 & 7.7 & 0.99 & 1.01 \\
\hline & lens & 17.9 & 1.00 & 0.99 & 12.0 & 1.01 & 1.06 \\
\hline & lac. gld. & 6.5 & 0.96 & 1.18 & 3.7 & 0.98 & 1.26 \\
\hline \multirow{4}{*}{$\# 4$} & fovea & 12.7 & 0.99 & 0.98 & 7.7 & 1.00 & 1.03 \\
\hline & opt. dsc. & 12.8 & 1.00 & 1.00 & 7.8 & 1.00 & 1.02 \\
\hline & lens & 17.9 & 1.00 & 0.99 & 12.0 & 1.01 & 1.06 \\
\hline & lac. gld. & 5.7 & 1.00 & 1.00 & 2.9 & 1.00 & 1.03 \\
\hline \multirow{4}{*}{$\# 5$} & fovea & 58.7 & 1.00 & 1.00 & 45.3 & 1.00 & 1.09 \\
\hline & opt. dsc. & 23.0 & 1.00 & 0.97 & 15.1 & 1.00 & 1.01 \\
\hline & lens & 10.1 & 1.00 & 0.97 & 6.0 & 1.01 & 1.00 \\
\hline & lac. gld. & 9.1 & 1.00 & 1.02 & 4.8 & 1.00 & 1.02 \\
\hline \multirow{4}{*}{$\# 6$} & fovea & 20.4 & 1.00 & 0.96 & 13.2 & 1.00 & 1.01 \\
\hline & opt. dsc. & 45.7 & 1.00 & 0.99 & 33.8 & 1.00 & 1.05 \\
\hline & lens & 13.2 & 1.01 & 0.99 & 8.3 & 1.00 & 1.01 \\
\hline & lac. gld. & 5.1 & 1.00 & 1.00 & 2.6 & 1.01 & 1.10 \\
\hline \multirow{4}{*}{$\# 7$} & fovea & 58.7 & 1.00 & 1.00 & 45.3 & 1.00 & 1.09 \\
\hline & opt. dsc. & 47.1 & 1.00 & 0.97 & 33.8 & 1.00 & 1.00 \\
\hline & lens & 10.1 & 1.00 & 0.97 & 6.0 & 1.01 & 1.00 \\
\hline & lac. gld. & 9.1 & 1.00 & 0.98 & 4.9 & 1.00 & 1.03 \\
\hline \multirow{4}{*}{$\# 8$} & fovea & 58.7 & 1.00 & 1.00 & 45.3 & 1.00 & 1.09 \\
\hline & opt. dsc. & 47.0 & 1.00 & 0.97 & 33.7 & 1.00 & 1.01 \\
\hline & lens & 10.1 & 1.00 & 0.97 & 6.0 & 1.01 & 1.00 \\
\hline & lac. gld. & 4.9 & 1.00 & 1.00 & 2.4 & 1.00 & 1.04 \\
\hline
\end{tabular}


Comparisons between egs_brachy and BrachyDose 3D dose distributions using a COMS $16 \mathrm{~mm}$ eye plaque for both ${ }^{125} \mathrm{I}$ and ${ }^{103} \mathrm{Pd}$ seeds are given in Figure 3.5. These comparisons are in the form of $\Delta \mathrm{D}_{L O C A L}$ and $\Delta \mathrm{D}_{G L O B A L}$ discussed in Section 2.4. For ${ }^{125} \mathrm{I}, \triangle \mathrm{D}_{L O C A L}$ is not strongly skewed but is shifted as to be centered at $-0.6 \%$, showing a small systematic difference between the two codes. $\triangle \mathrm{D}_{G L O B A L}$ shows a skew, but is peaked at $-0.1 \%$. For ${ }^{103} \mathrm{Pd}$ there is a larger skew in $\triangle \mathrm{D}_{L O C A L}$ than for ${ }^{125} \mathrm{I}$, the peak is at $\sim 0.5 \%$ showing a similar systematic difference but in the opposite direction. $\triangle \mathrm{D}_{G L O B A L}$ shows a smaller skew than for ${ }^{125} \mathrm{I}$, the natural peak seems to be at $0.02 \%$, but a sharp single bin peak occurs at $0 \%$. These comparisons show a small overall difference between the two codes that is discussed in Chapter 4 .

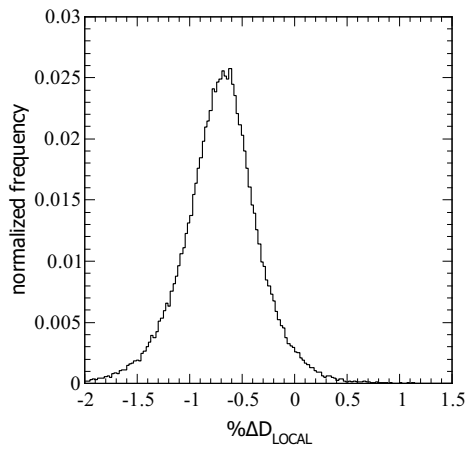

(a)

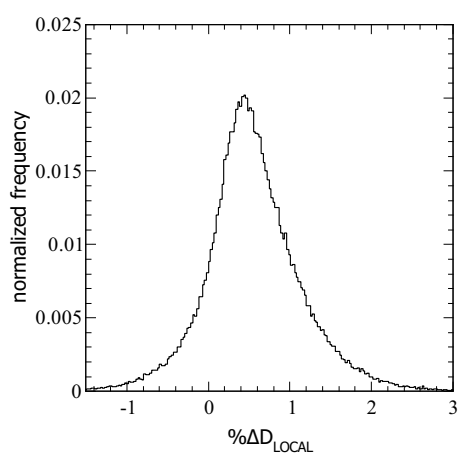

(c)

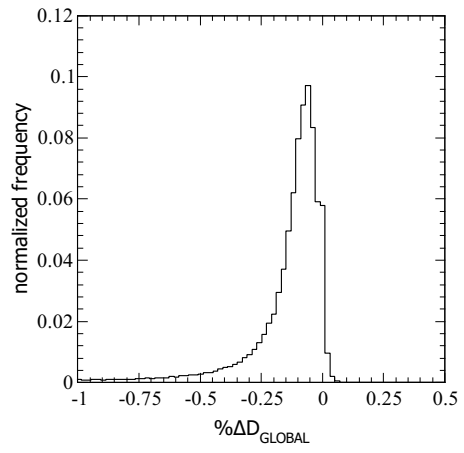

(b)

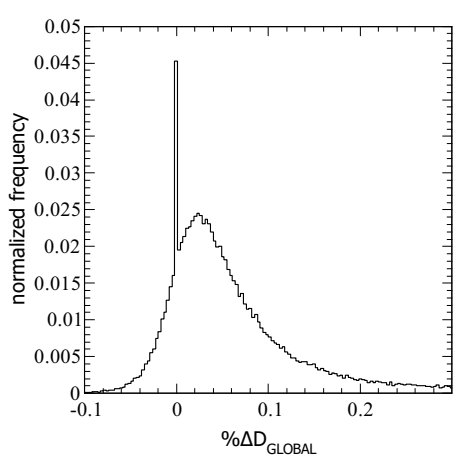

(d)

Figure 3.5: A 3D $D_{w, w}$ comparison between egs_brachy and BrachyDose. Subfigures (a) and (b) were obtained using ${ }^{125}$ I seeds. Subfigures (c) and (d) were obtained using ${ }^{103} \mathrm{Pd}$ seeds. 


\subsubsection{Comparison of $D_{T G 43}, D_{w, w}$, and $D_{m, m}$}

Figure 3.6 shows isodose contours for different photon plaque models, using COMS $16 \mathrm{~mm}$ as a reference for the COMS standard. Profiles of $D_{T G 43}, D_{w, w}$, and $D_{m, m}$ are shown for each of the COMS $16 \mathrm{~mm}$, "COMS - thin acrylic", "short lip - acrylic", "no lip - silastic", and "stainless steel - acrylic" plaques. These isodose contours are scaled such that $D_{T G 43}$ is 85 Gy at the tumor apex $((0.25,0.25,-0.675) \mathrm{cm}$ in eye centre coordinates). It is shown that $D_{T G 43}$ is consistently higher than $D_{w, w}$ or $D_{m, m}$. In contrast, $D_{m, m}$ varies between being higher than $D_{w, w}$ and lower than $D_{w, w}$ depending on the medium being traversed, but is still generally lower than $D_{T G 43}$.

Figure 3.7 gives plaque central axis dose ratios for $D_{w, w}$ and $D_{m, m}$ over $D_{T G 43}$. For the purposes of comparison to Lesperance et al. ${ }^{52}$, the ratio of egs_brachy calculated $D_{w, w}$ over $D_{T G 43}$ is shown alongside their equivalent BrachyDose results. Relative differences in the ratios appear to be statistical and are between $0.4 \%$ and $0.8 \%$ up to the tumor apex and between $1.6 \%$ and $2.3 \%$ up to the opposite side of the eye. The smallest difference up to the tumor is for the "no lip - silastic" plaque and the largest differences are the COMS $16 \mathrm{~mm}$ and "stainless steel - acrylic". Up to the opposite side of the eye the smallest difference is for the "short lip - acrylic" plaque and the largest is for the "COMS - thin acrylic" plaque.

Minimum, maximum, and average $D_{T G 43}, D_{w, w}$, and $D_{m, m}$ in different eye struc-

tures for a COMS $16 \mathrm{~mm}$ plaque loaded with ${ }^{125} \mathrm{I}$ seeds are given in Table 3.3. This table also includes the ratios of $D_{w, w}$ and $D_{m, m}$ with $D_{T G 43}$ for those eye structures. 


$$
\frac{p 2}{D}
$$




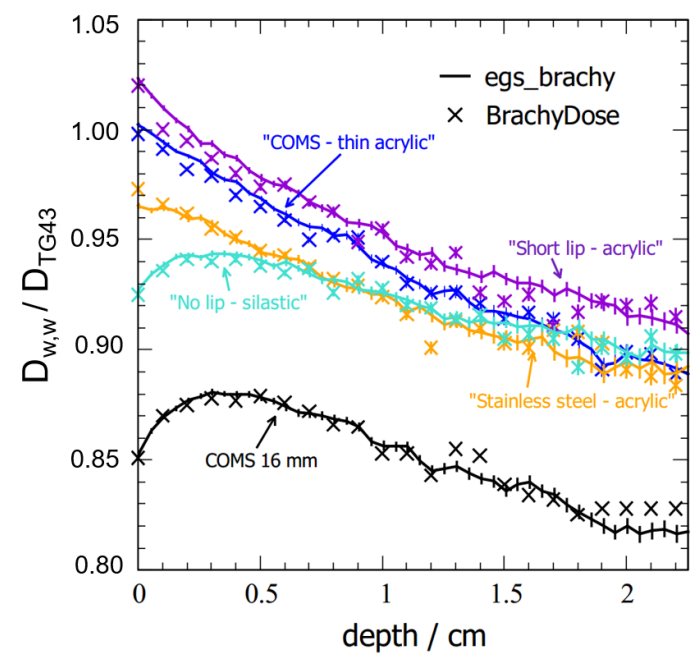

(a)

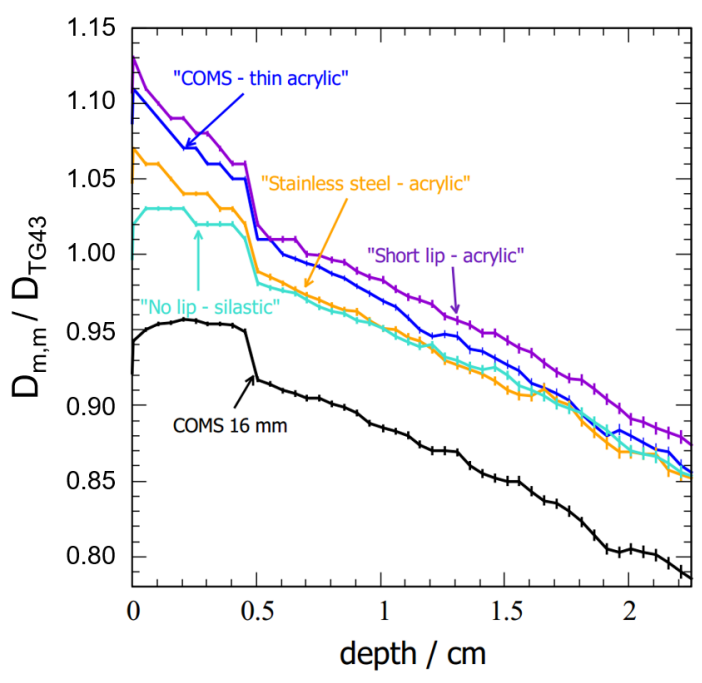

(b)

Figure 3.7: Dose ratios are given in (a) $D_{w, w} / D_{T G 43}$, and (b) $D_{m, m} / D_{T G 43}$ for five different plaque models fully loaded with ${ }^{125}$ I seeds: COMS $16 \mathrm{~mm}$, "COMS thin acrylic", "short lip - acrylic", "no lip - silastic", and "stainless steel - acrylic". All plaques are loaded with ${ }^{125}$ I seeds. 
Table 3.3: $D_{T G 43}, D_{w, w}$, and $D_{m, m}$ to structures in the eye (the lens, tumor, sclera, optic nerve, vitreous fluid, aqueous humor, iris, and cornea) using a COMS $16 \mathrm{~mm}$ plaque model loaded with ${ }^{125}$ I seeds.

\begin{tabular}{|c|c|c|c|c|c|c|}
\hline $\begin{array}{c}\text { eye } \\
\text { struct. }\end{array}$ & value & $D_{T G 43}$ & $D_{w, w}$ & $D_{m, m}$ & $\frac{D_{w, w}}{D_{T G 43}}$ & $\frac{D_{m, m}}{D_{T G 43}}$ \\
\hline \multirow[t]{3}{*}{ lens } & Min & 12.80 & 10.40 & 8.74 & 0.81 & 0.68 \\
\hline & Max & 39.34 & 32.41 & 28.61 & 0.82 & 0.73 \\
\hline & Avg & 22.45 & 18.42 & 16.01 & 0.82 & 0.71 \\
\hline \multirow[t]{3}{*}{ tumor } & Min & 92.19 & 80.94 & 87.30 & 0.88 & 0.95 \\
\hline & Max & 289.69 & 247.89 & 274.78 & 0.86 & 0.95 \\
\hline & Avg & 169.40 & 147.03 & 160.96 & 0.87 & 0.95 \\
\hline \multirow[t]{3}{*}{ sclera } & Min & 6.44 & 5.23 & 4.07 & 0.81 & 0.63 \\
\hline & Max & 337.25 & 283.25 & 250.47 & 0.84 & 0.74 \\
\hline & Avg & 38.37 & 30.34 & 26.25 & 0.79 & 0.68 \\
\hline \multirow{3}{*}{$\begin{array}{l}\text { opt. } \\
\text { nrv. }\end{array}$} & Min & 8.05 & 1.29 & 1.36 & 0.16 & 0.17 \\
\hline & Max & 24.70 & 18.06 & 17.85 & 0.73 & 0.72 \\
\hline & Avg & 13.86 & 6.17 & 6.18 & 0.45 & 0.45 \\
\hline \multirow[t]{3}{*}{ vitreous } & Min & 6.74 & 5.49 & 5.21 & 0.81 & 0.77 \\
\hline & Max & 180.22 & 155.01 & 165.55 & 0.86 & 0.92 \\
\hline & Avg & 29.38 & 24.43 & 25.30 & 0.83 & 0.86 \\
\hline \multirow[t]{3}{*}{ aqueous } & Min & 8.94 & 7.20 & 6.69 & 0.81 & 0.75 \\
\hline & Max & 61.14 & 48.59 & 50.61 & 0.79 & 0.83 \\
\hline & Avg & 20.08 & 15.78 & 15.74 & 0.79 & 0.78 \\
\hline \multirow[t]{3}{*}{ iris } & Min & 9.82 & 7.88 & 7.20 & 0.80 & 0.73 \\
\hline & Max & 41.24 & 32.17 & 32.45 & 0.78 & 0.79 \\
\hline & Avg & 20.00 & 15.76 & 15.36 & 0.79 & 0.77 \\
\hline \multirow[t]{3}{*}{ cornea } & Min & 9.77 & 7.74 & 6.15 & 0.79 & 0.63 \\
\hline & Max & 31.15 & 19.64 & 17.58 & 0.63 & 0.56 \\
\hline & Avg & 15.95 & 11.52 & 9.78 & 0.72 & 0.61 \\
\hline
\end{tabular}




\subsection{Beta plaque results}

Doses calculated in egs_brachy using ${ }^{106} \mathrm{Ru}$ beta plaques are presented in several different forms. First, Figure 3.8 shows depth-dose curves along the plaque central axis. These curves are presented alongside doses calculated with PENELOPE by Hermida-Lopez ${ }^{47}$ and are given for eight different plaque varieties(with geometries discussed in Section 2.1.2): CCA, CCB, CCC, CCD, CGD, CXS, CCX-Y-Z, and a simplified planar model of the CCB plaque. These depth-dose curves give $D_{w, w}$ and are scaled to $100 \%$ at the point $(0,0,0.1) \mathrm{cm}$ in the eye plaque coordinate system, the same scaling used in the PENELOPE paper ${ }^{47}$. The relative dose difference between egs_brachy and PENELOPE at depths up to the tumor apex is less than $2.2 \%$ for the CXS plaque. All other plaques have relative dose differences less than $1.4 \%$. The relative dose differences get substantially larger past the tumor where doses are low (less than $10 \mathrm{~Gy}$ ) and reach up to $13 \%$. The best agreement is with the CCB plaque, which has a relative dose difference of less than $4.5 \%$ up to $1 \mathrm{~cm}$.

Next, Figure 3.9 is a central axis depth-dose curve for the CCB plaque given in units of pGy per decay. The pGy per decay depth-dose curve is presented alongside doses calculated by Sommer et al. ${ }^{49}$ using a Monte Carlo code known as GEANT4 and a reference dataset from the $\mathrm{BEBIG}{ }^{106} \mathrm{Ru}$ beta plaque user manual that is averaged over 174 measurements and has a combined uncertainty of $20 \%^{90}$. Comparing pGy per decay for egs_brachy and GEANT4 there is at most a $0.7 \%$ disagreement up to the tumor apex. Up to $1 \mathrm{~cm}$, the largest disagreement is $4.4 \%$. Comparing egs_brachy doses to the BEBIG reference dataset shows a disagreement of up $7.5 \%$ at a depth of $1 \mathrm{~cm}$.

For each plaque type, dose profiles of an axis lateral to the central axis are shown in Figures 3.10 and 3.11. These profiles are given at different depths along the $\mathrm{z}$ axis 
ranging from $0.1 \mathrm{~cm}$ to $0.8 \mathrm{~cm}$ in the eye plaque coordinate system. The profiles are presented alongside PENELOPE results from the same work as the depth-dose curves $^{47}$ and are scaled to $100 \%$ at the same point $(0,0,0.1) \mathrm{cm}$. Looking at the lateral profiles at different depths most relative dose differences are less than $3 \%$. Higher relative differences are found at locations in the penumbra region of the plaque, at far depths, or where they intersect the plaque. 

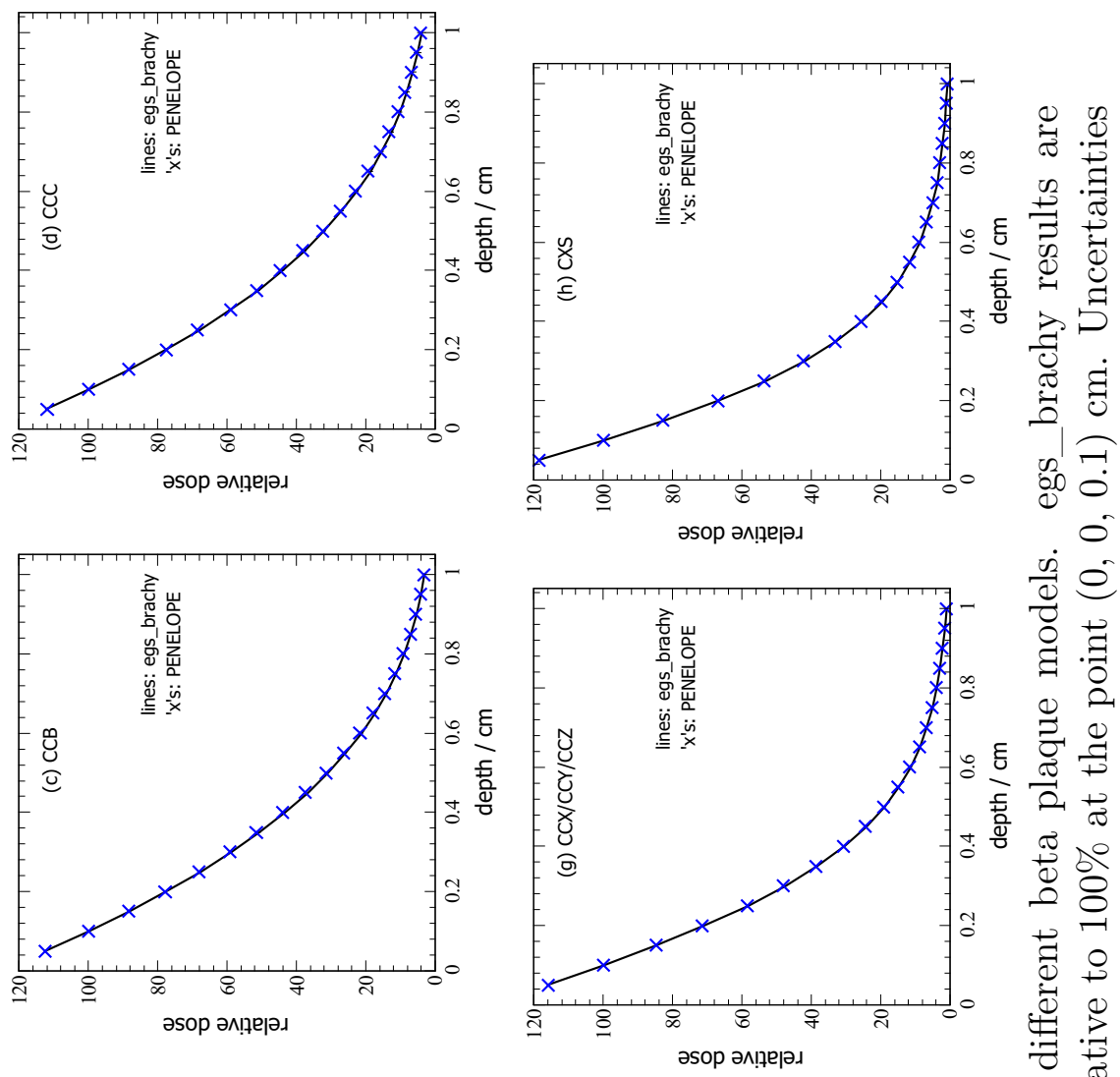

$\dot{\Omega} 0$

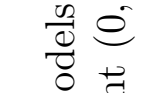

芩.

?

E

吾

要

궁

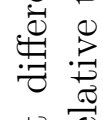
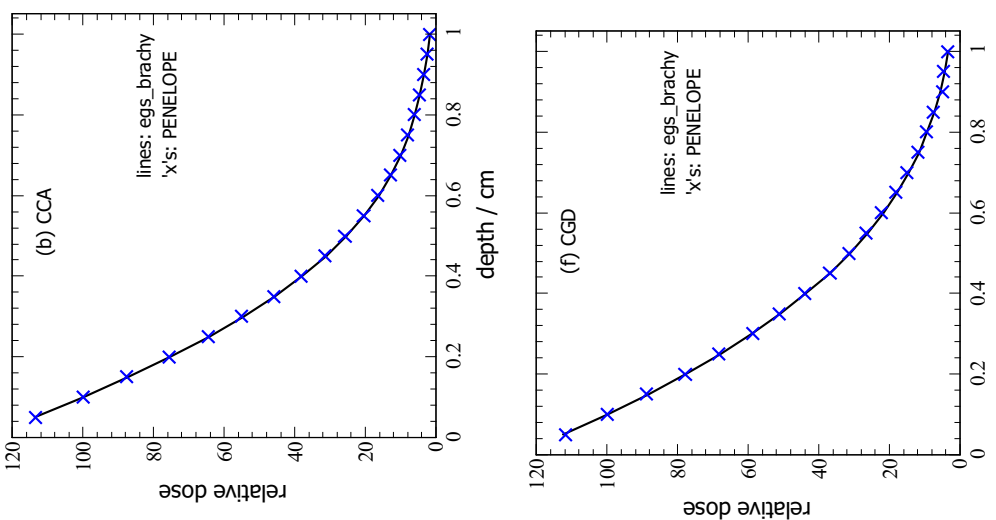

$\infty 0$

.

등 巳

흠

$\begin{array}{lll}0 & 0 \\ 0 & 0 \\ 0 & 0\end{array}$

I.

过完
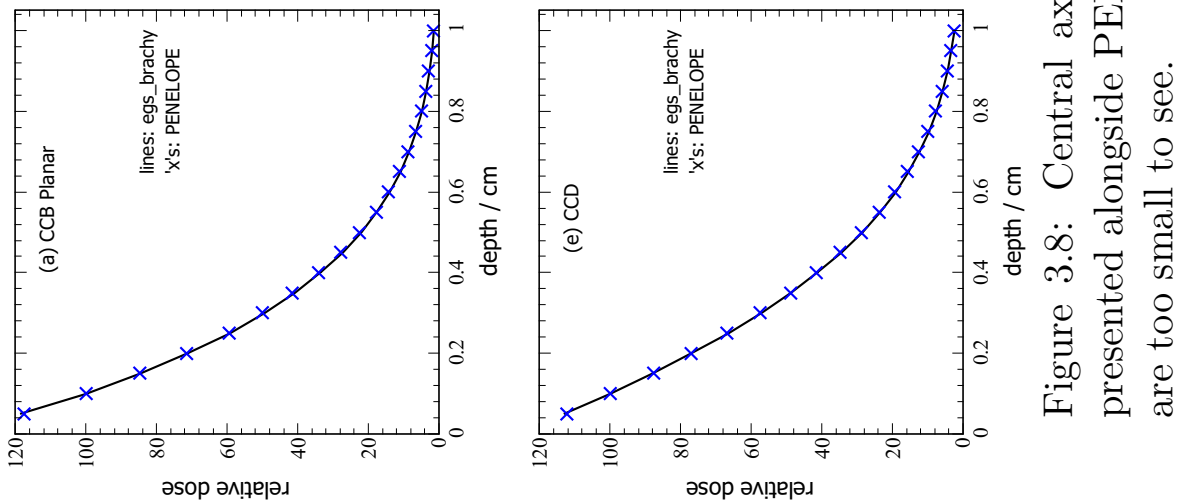


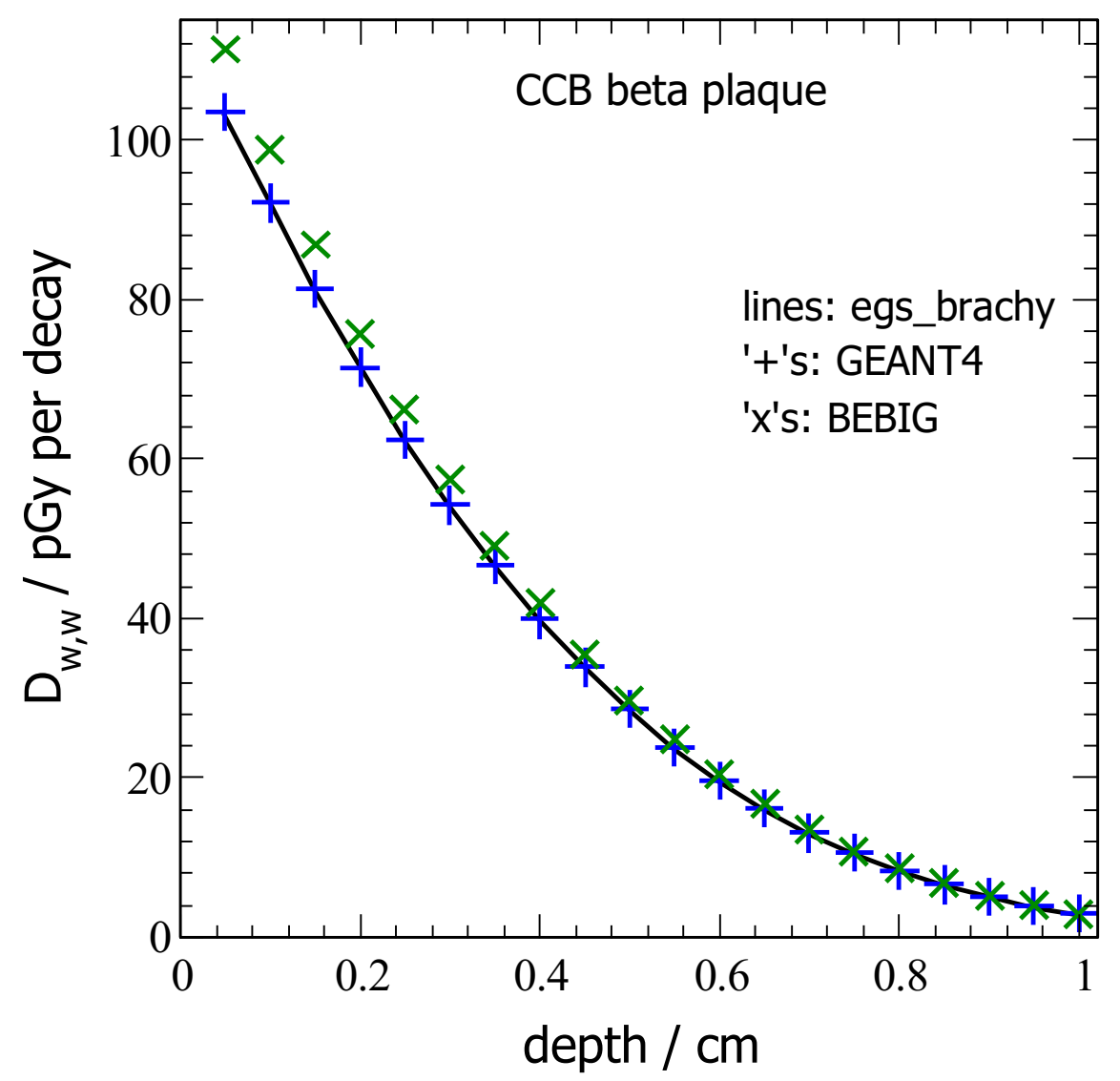

Figure 3.9: A comparison of the central axis dose per decay with depth between egs_brachy, GEANT4, and an averaged dataset from BEBIG certificates. Uncertainties are too small to see. 


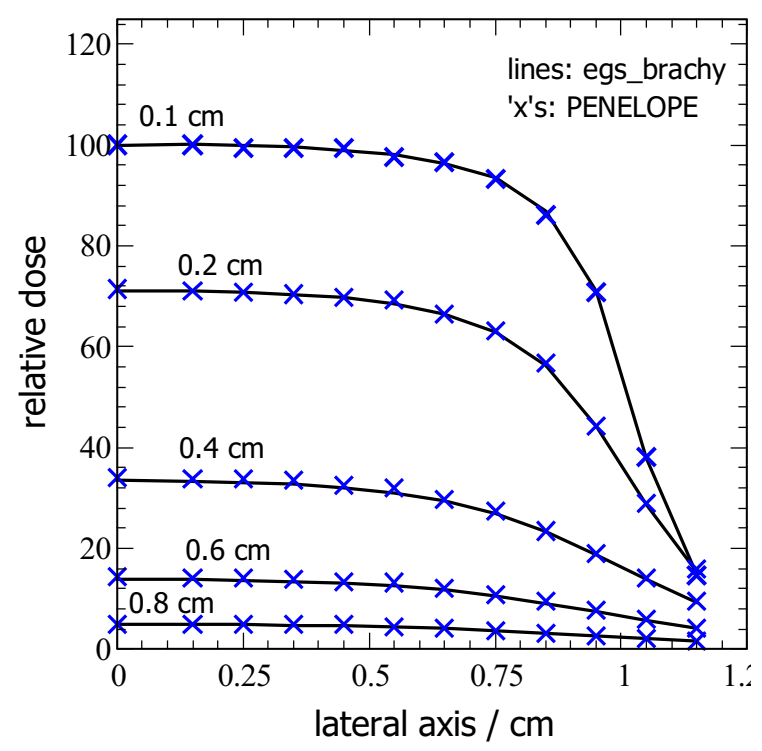

(a) CCB Planar

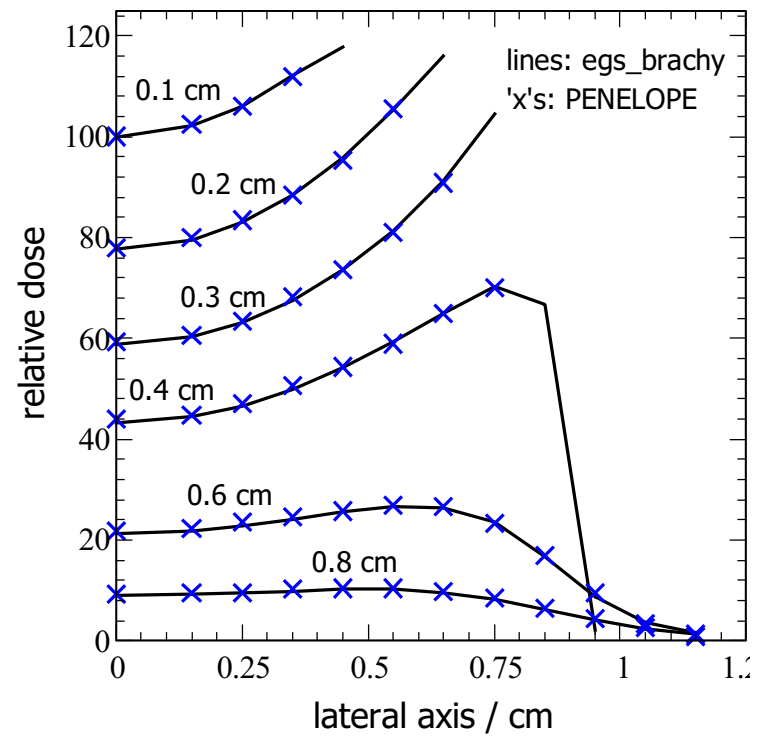

(c) $\mathrm{CCB}$

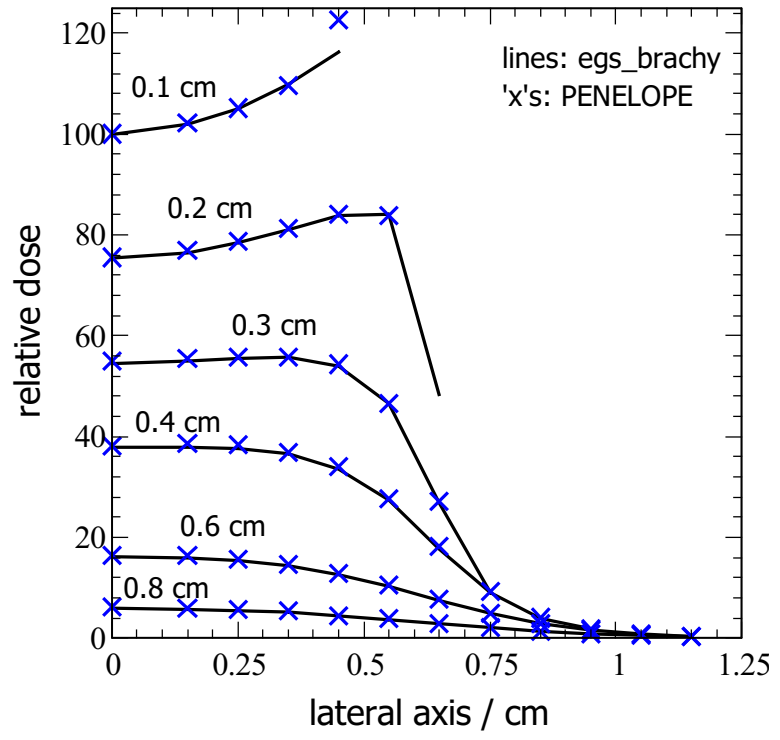

(b) CCA

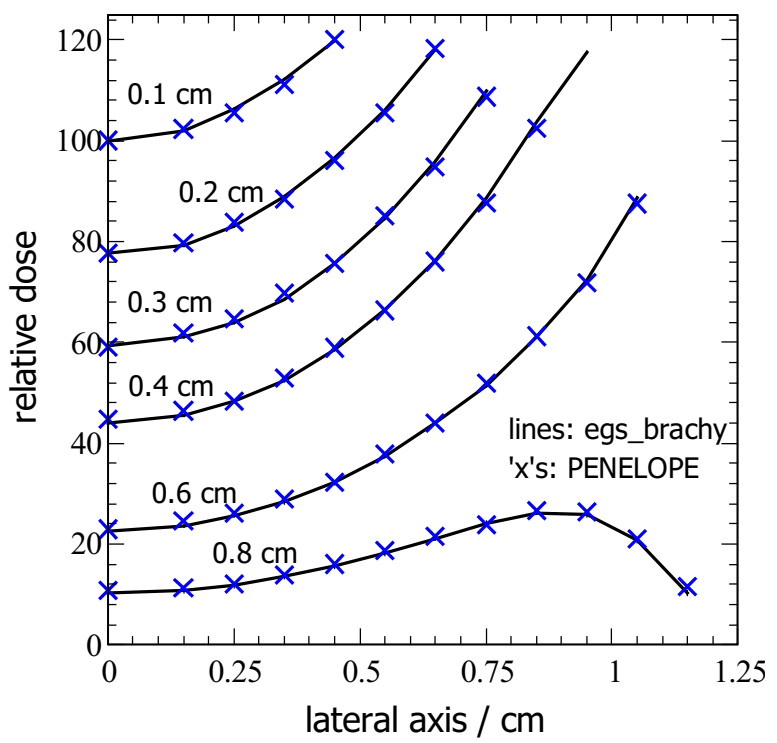

(d) $\mathrm{CCC}$

Figure 3.10: Dose profiles at different depths comparing egs_brachy and PENELOPE. Doses are relative to $100 \%$ at the point $(0,0,0.1) \mathrm{cm}$. Uncertainties are too small to see. 


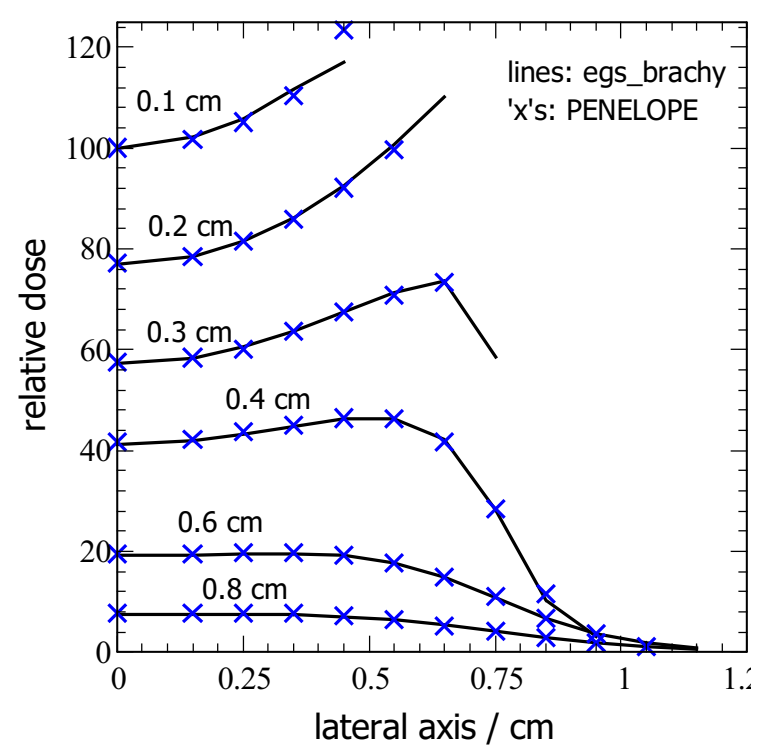

(a) CCD

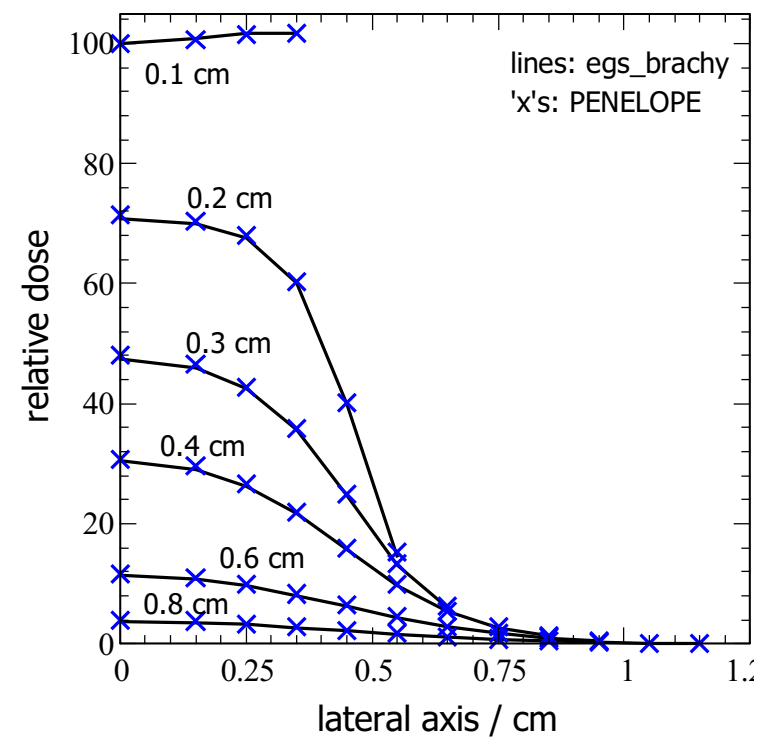

(c) CCX-Y-Z

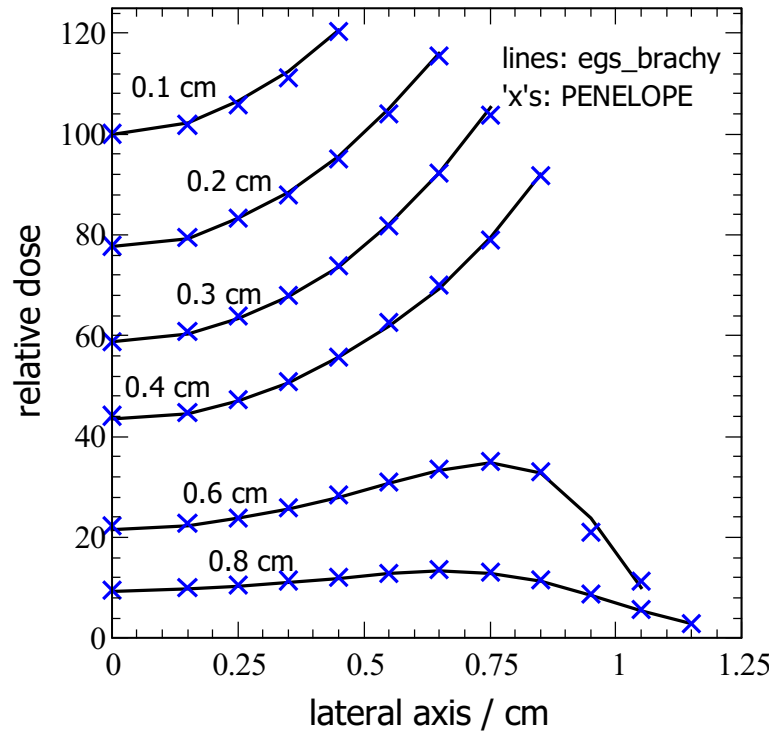

(b) CGD

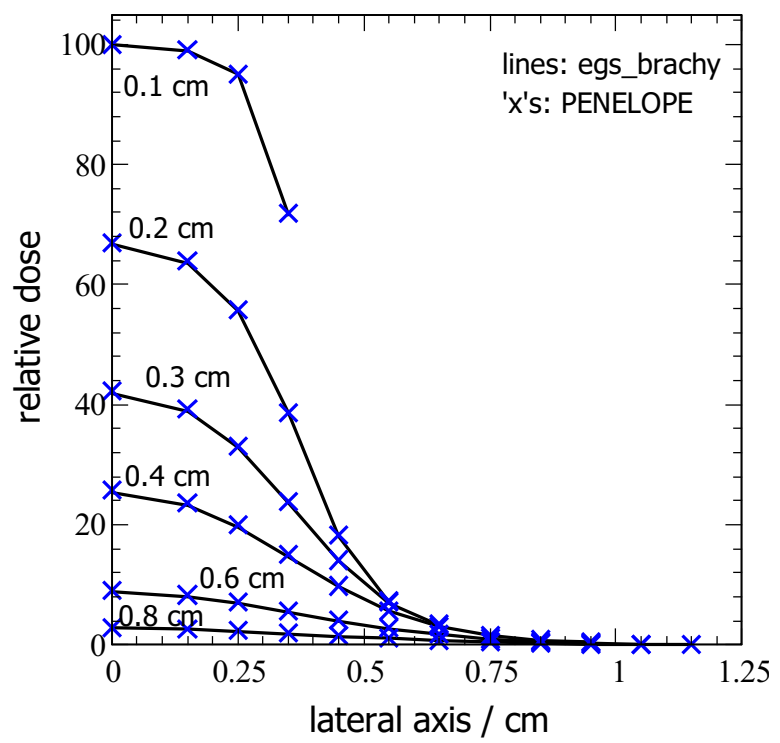

(d) CXS

Figure 3.11: Dose profiles at different depths comparing egs_brachy and PENELOPE. Doses are relative to $100 \%$ at the point $(0,0,0.1) \mathrm{cm}$. Uncertainties are too small to see. 


\subsubsection{Comparison of CIA beta plaque models}

Some of the beta plaques have a cutout notch to fit around structures of the eye. Two versions of the CIA beta plaque are simulated here, CIA-HL and CIA. For a definition of these two plaque geometries refer to Section 2.1.2. Figure 3.12 presents dose distribution for the two models alongside the PENELOPE doses for the same plaque calculated by Hermida-Lopez ${ }^{47}$. For the majority of points both central axis depth-dose curve and $\mathrm{x}$-axis profiles have very good agreement (within a disagreement of only $0.1 \%$ ) between the CIA-HL and the CIA. There are a few points where they have a $1-3 \%$ disagreement and even fewer with quite large disagreement (20\% or greater). All of these points are located at far depths or further out on the xaxis or both. The points of greatest disagreement are shown in Figure 3.13, where isodose contours of CIA-Hl and CIA are given. The isodose contours in this figure show large discrepancies near the plaque on the positive $\mathrm{x}$-axis. When comparing to PENELOPE, both versions of the egs_brachy CIA plaque result in doses in good agreement (2\% or less disagreement) for most points, only at further depths and distances along the $\mathrm{x}$-axis do the disagreements reach $3 \%-6 \%$. 

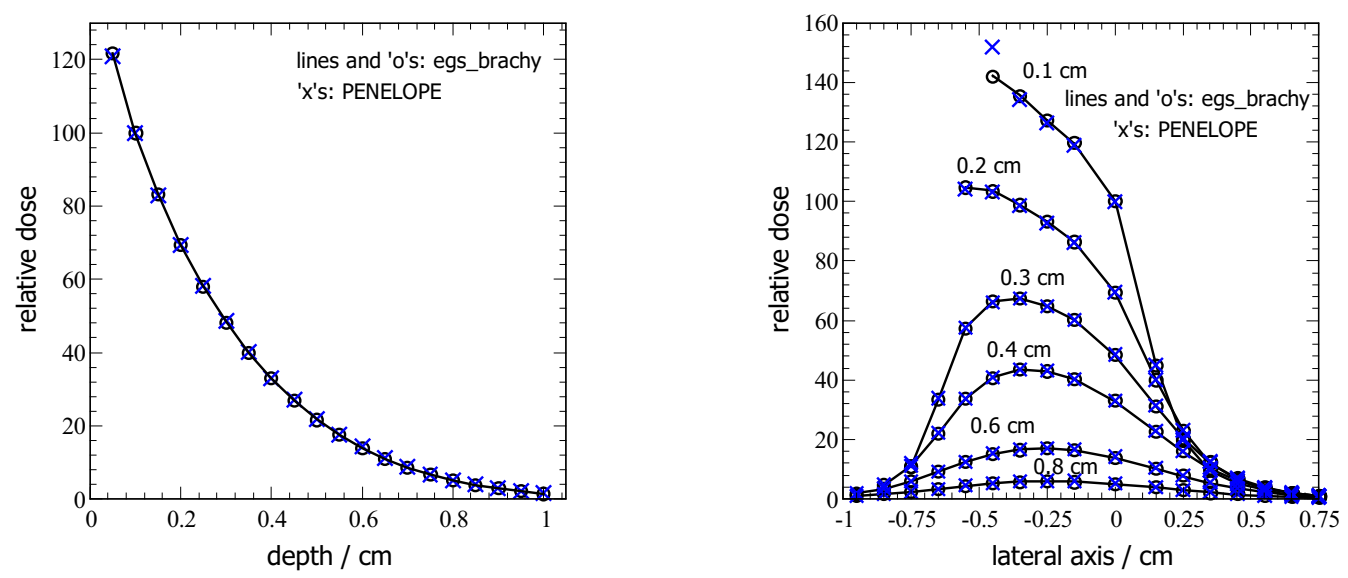

Figure 3.12: Plots showing doses using different CIA models. Lines give the egs_brachy doses using the CIA-HL model, 'o's give the egs_brachy doses using the CIA model, and ' $x$ 's give the doses calculated with PENELOPE by HermidaLopez $^{47}$. (a) Shows the depth-dose curve along the plaque central axis. (b) Shows dose profiles along the x-axis (where the cutout notch is located) at different depths of the central axis. Uncertainties are too small to see.

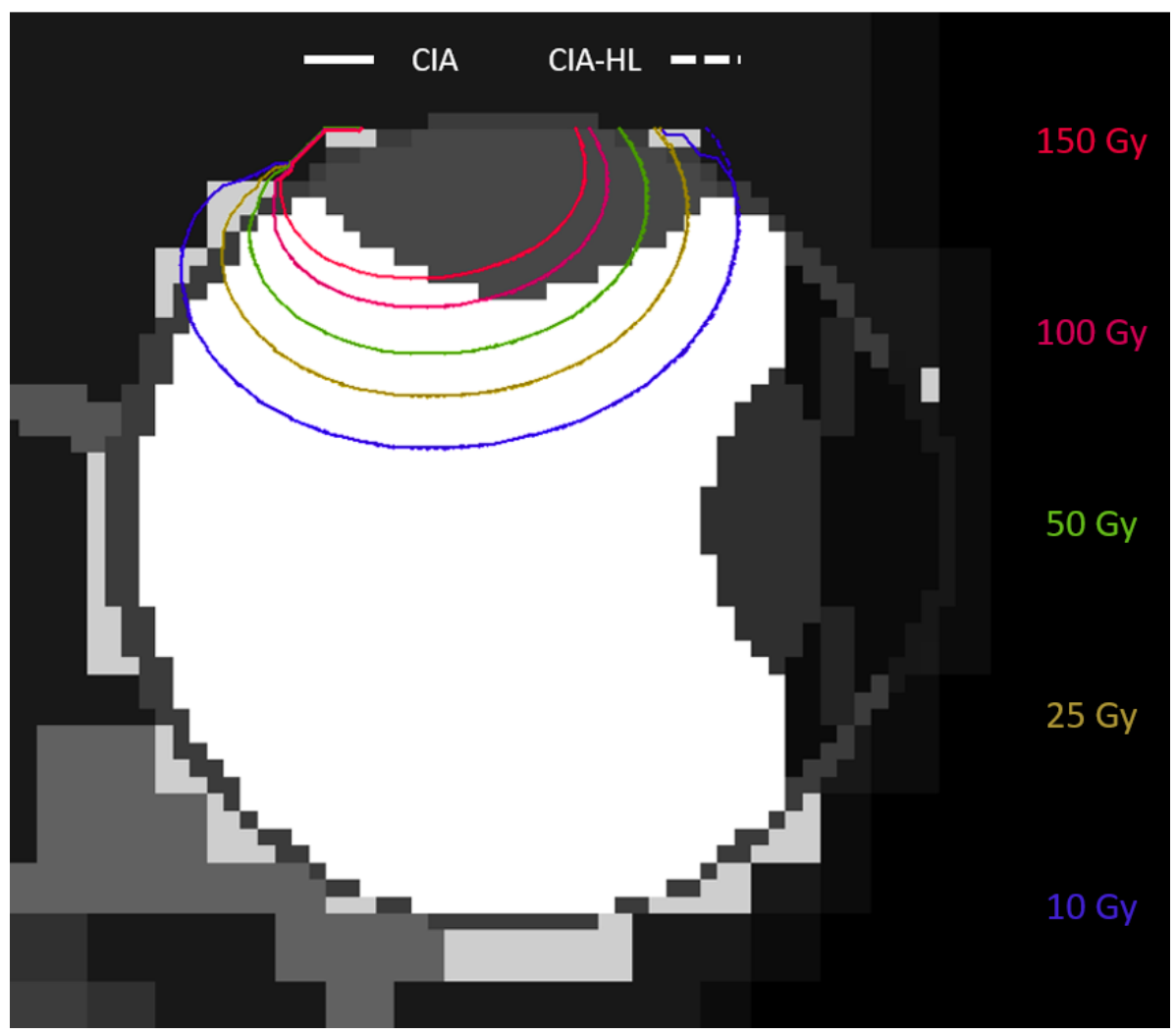

Figure 3.13: isodose contours for the two models, CIA and CIA-HL. Solid lines are CIA, dashed lines are CIA-HL. 


\subsection{Photon and beta plaque comparisons}

To compare a photon-emitting plaque to a beta-emitting plaque, the COMS $16 \mathrm{~mm}$ and CCB plaques were chosen for their similar size and as commonly-used and studied designs of the two plaques types. Subfigures (a) and (b) of Figure 3.14 show a comparison of plaque central axis depth-dose curves for $D_{w, w}$ and $D_{m, m}$ respectively. Figure 3.15 shows isodose contours of $D_{w, w}$ and $D_{m, m}$ for both the photon plaque and the beta plaque. Both Figures show generally higher doses near to the plaque for the beta plaque than for the photon plaque. Table 3.4 gives the dose $D_{w, w}$ at the points of interest laid out in Section 2.4 for both plaque types and shows much lower doses to the points of interest for the beta plaque in most cases. Table 3.5 gives minimum, maximum, and average doses to ocular structures within the eye for

both plaque types and both $D_{w, w}$ and $D_{m, m}$, as well as a ratio between $D_{m, m}$ and $D_{w, w}$ for the beta plaque to highlight the differences caused by introducing the eye phantom. 


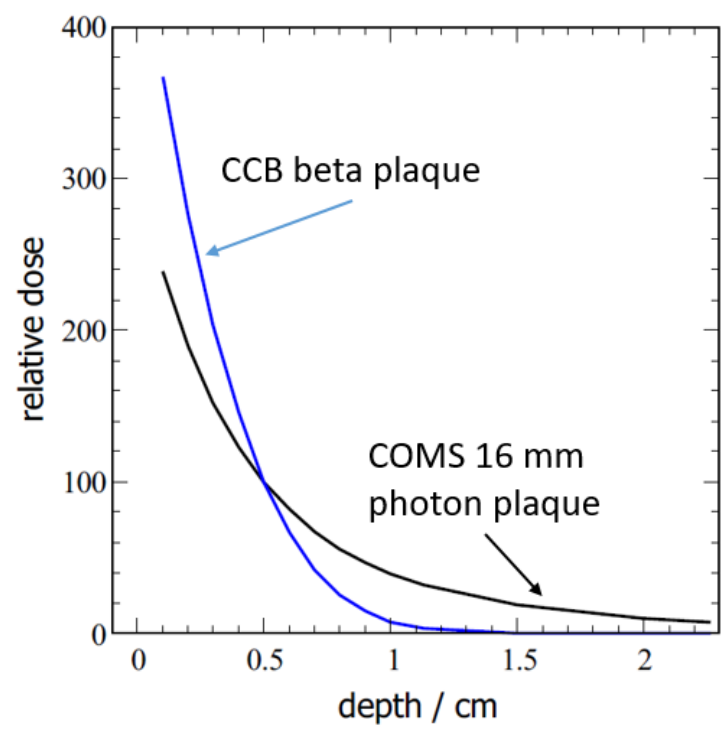

(a)

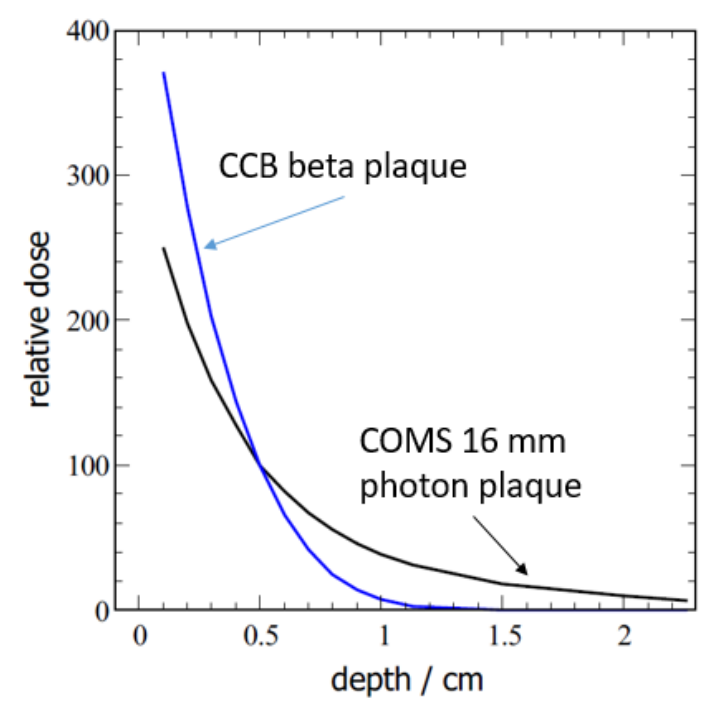

(b)

Figure 3.14: Depth-dose curves for both the COMS $16 \mathrm{~mm}$ photon-emitting plaque and the CCB beta-emitting plaque, (a) gives $D_{w, w}$, and (b) $D_{m, m}$. Uncertainties are too small to see. 


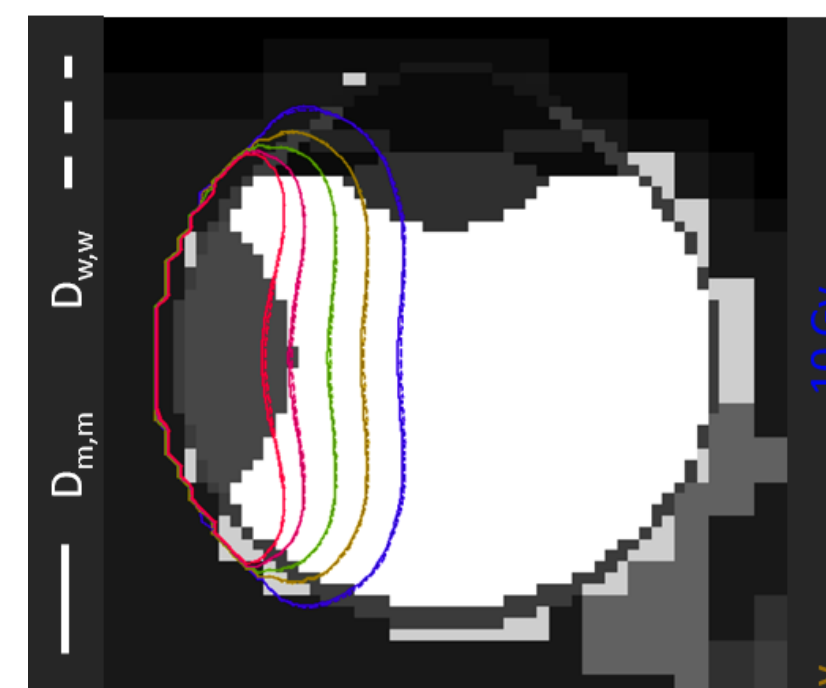

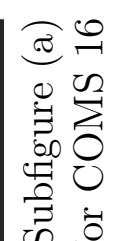

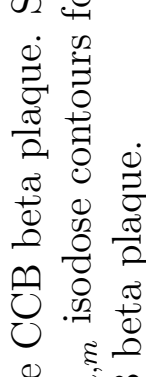

झ हीं

(1)
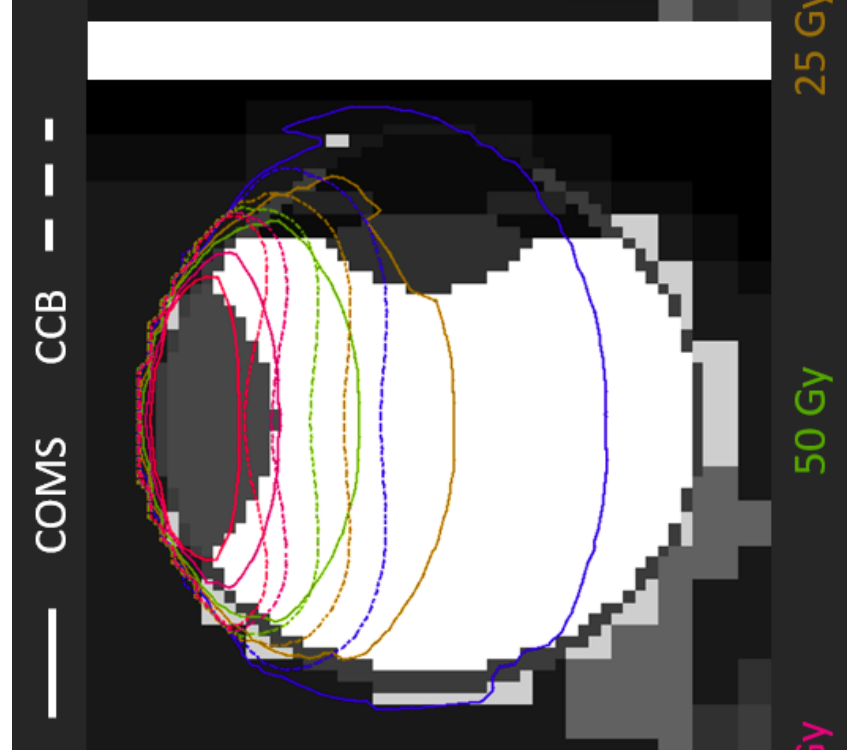

ซี

ช.

完 2

리류

ชี

슬 全

घี $\stackrel{0}{0}$

व ह ह

का है

$\sum_{0}^{\infty} \oplus$

$\circlearrowright \circlearrowright$ ठี

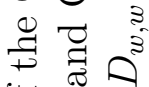

फै घ

잉

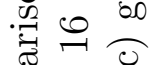

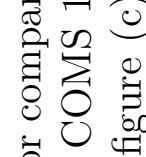

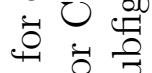

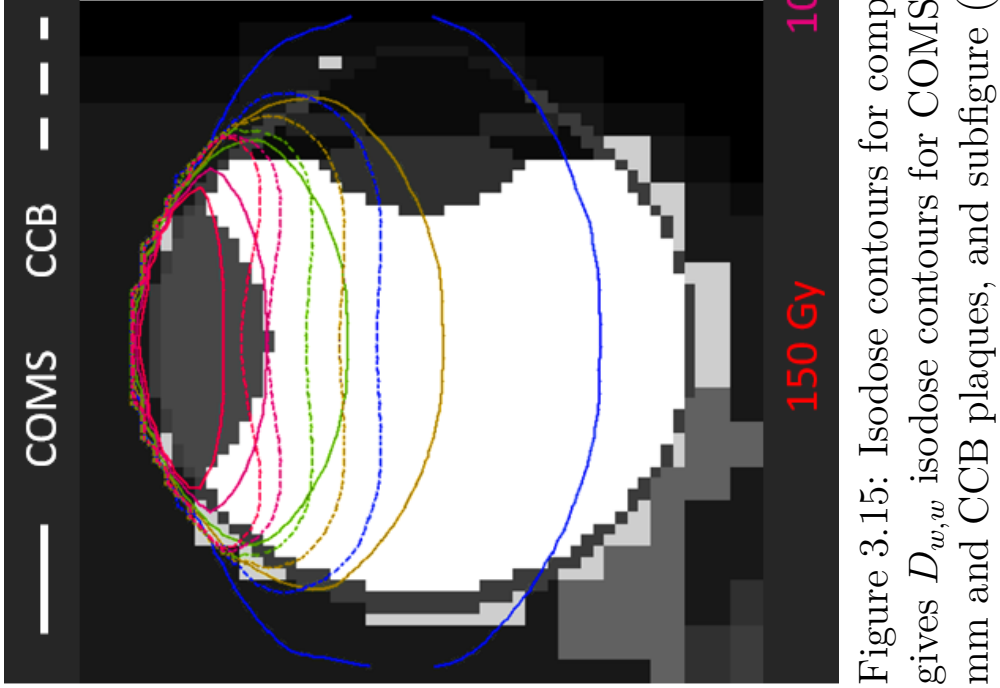


Table 3.4: $D_{w, w}$ calculated using a COMS $16 \mathrm{~mm}$ plaque loaded with ${ }^{125} \mathrm{I}$ seeds and a CCB plaque at different plaque locations and points of interest. $D_{w, w}$ is scaled to $100 \%$ at the tumor apex $(\mathrm{X}, \mathrm{Y}, \mathrm{Z})=(0.25,0.25,-0.675) \mathrm{cm}$.

\begin{tabular}{|c|c|c|c|c|}
\hline $\begin{array}{l}\text { plq. } \\
\text { pos. }\end{array}$ & $\begin{array}{l}\text { point of } \\
\text { interest }\end{array}$ & $\begin{array}{l}\text { COMS } \\
16 \mathrm{~mm}\end{array}$ & $\mathrm{CCB}$ & $\frac{D_{C C B}}{D_{C O M S}}$ \\
\hline \multirow{4}{*}{$\# 1$} & \multirow{4}{*}{$\begin{array}{c}\text { fovea } \\
\text { opt. dsc. } \\
\text { lens } \\
\text { lac. gld. }\end{array}$} & 17.06 & 1.08 & 0.06 \\
\hline & & 12.11 & 0.19 & 0.02 \\
\hline & & 24.08 & 2.99 & 0.12 \\
\hline & & 8.9 & 3.35 & 0.38 \\
\hline \multirow{4}{*}{$\# 2$} & \multirow{4}{*}{$\begin{array}{c}\text { fovea } \\
\text { opt. dsc. } \\
\text { lens } \\
\text { lac. gld. }\end{array}$} & 17.06 & 1.08 & 0.06 \\
\hline & & 27.92 & 9.81 & 0.35 \\
\hline & & 24.08 & 2.99 & 0.12 \\
\hline & & 7.59 & 0.11 & 0.01 \\
\hline \multirow{4}{*}{$\# 3$} & \multirow{4}{*}{$\begin{array}{c}\text { fovea } \\
\text { opt. dsc. } \\
\text { lens } \\
\text { lac. gld. }\end{array}$} & 17.06 & 1.08 & 0.06 \\
\hline & & 17.19 & 1.06 & 0.06 \\
\hline & & 24.08 & 2.99 & 0.12 \\
\hline & & 8.7 & 3.28 & 0.38 \\
\hline \multirow{4}{*}{$\# 4$} & \multirow{4}{*}{$\begin{array}{c}\text { fovea } \\
\text { opt. dsc. } \\
\text { lens } \\
\text { lac. gld. }\end{array}$} & 17.06 & 1.08 & 0.06 \\
\hline & & 17.23 & 1.07 & 0.06 \\
\hline & & 24.08 & 2.99 & 0.12 \\
\hline & & 7.68 & 0.11 & 0.01 \\
\hline \multirow{4}{*}{$\# 5$} & \multirow{4}{*}{$\begin{array}{c}\text { fovea } \\
\text { opt. dsc. } \\
\text { lens } \\
\text { lac. gld. }\end{array}$} & 79.02 & 263.75 & 3.34 \\
\hline & & 31.01 & 14.25 & 0.46 \\
\hline & & 13.65 & 0.18 & 0.01 \\
\hline & & 12.25 & 0.4 & 0.03 \\
\hline \multirow{4}{*}{$\# 6$} & \multirow{4}{*}{$\begin{array}{c}\text { fovea } \\
\text { opt. dsc. } \\
\text { lens } \\
\text { lac. gld. }\end{array}$} & 27.44 & 9.24 & 0.34 \\
\hline & & 61.42 & 132.38 & 2.16 \\
\hline & & 17.84 & 0.54 & 0.03 \\
\hline & & 6.9 & 0.11 & 0.02 \\
\hline \multirow{4}{*}{$\# 7$} & \multirow{4}{*}{$\begin{array}{c}\text { fovea } \\
\text { opt. dsc. } \\
\text { lens } \\
\text { lac. gld. }\end{array}$} & 79.02 & 263.75 & 3.34 \\
\hline & & 63.25 & 149.44 & 2.36 \\
\hline & & 13.65 & 0.18 & 0.01 \\
\hline & & 12.27 & 0.43 & 0.04 \\
\hline \multirow{4}{*}{$\# 8$} & \multirow{4}{*}{$\begin{array}{c}\text { fovea } \\
\text { opt. dsc. } \\
\text { lens } \\
\text { lac. gld. }\end{array}$} & 79.02 & 263.75 & 3.34 \\
\hline & & 63.27 & 149.57 & 2.36 \\
\hline & & 13.65 & 0.18 & 0.01 \\
\hline & & 6.56 & 0.1 & 0.02 \\
\hline
\end{tabular}


Table 3.5: Minimum, maximum, and average $D_{w, w}$ and $D_{m, m}$ for different eye structures (the lens, tumor, sclera, optic nerve, vitreous fluid, aqueous humor, iris, and cornea) calculated using both a COMS $16 \mathrm{~mm}$ plaque loaded with ${ }^{125} \mathrm{I}$ seeds and a CCB beta plaque. $D_{w, w}$ is scaled to $100 \%$ at the tumor apex $(\mathrm{X}, \mathrm{Y}, \mathrm{Z})=(0.25$, $0.25,-0.675) \mathrm{cm}, D_{m, m}$ is scaled using the same value.

\begin{tabular}{|c|c|c|c|c|c|c|}
\hline \multirow{2}{*}{$\begin{array}{c}\text { eye } \\
\text { struct. }\end{array}$} & \multirow[b]{2}{*}{ value } & \multicolumn{2}{|c|}{$D_{w, w}$} & \multicolumn{2}{|c|}{$D_{m, m}$} & \multirow{2}{*}{$\frac{\left(\frac{D_{m, m}}{D_{w, w}}\right)}{\mathrm{CCB}}$} \\
\hline & & COMS & CCB & COMS & CCB & \\
\hline \multirow[t]{3}{*}{ lens } & Min & 12.77 & 0.19 & 10.73 & 0.17 & 0.91 \\
\hline & Max & 39.79 & 30.05 & 35.13 & 29.58 & 0.98 \\
\hline & Avg & 22.62 & 4.62 & 19.66 & 4.39 & 0.95 \\
\hline \multirow[t]{3}{*}{ tumor } & Min & 99.37 & 99.83 & 107.18 & 93.88 & 0.94 \\
\hline & Max & 304.35 & 427.06 & 337.36 & 418.98 & 0.98 \\
\hline & Avg & 180.52 & 283.43 & 197.62 & 274.50 & 0.97 \\
\hline \multirow[t]{3}{*}{ sclera } & Min & 6.42 & 0.08 & 5.00 & 0.08 & 1.00 \\
\hline & Max & 347.77 & 463.11 & 307.52 & 454.73 & 0.98 \\
\hline & Avg & 37.26 & 58.61 & 32.23 & 57.56 & 0.98 \\
\hline \multirow{3}{*}{$\begin{array}{l}\text { opt. } \\
\text { nrv. }\end{array}$} & Min & 1.59 & 0.09 & 1.67 & 0.09 & 0.98 \\
\hline & Max & 22.17 & 4.97 & 21.91 & 4.60 & 0.93 \\
\hline & Avg & 7.58 & 0.67 & 7.59 & 0.62 & 0.93 \\
\hline \multirow[t]{3}{*}{ vitreous } & Min & 6.74 & 0.08 & 6.40 & 0.08 & 0.95 \\
\hline & Max & 190.32 & 402.32 & 203.25 & 400.69 & 1.00 \\
\hline & Avg & 30.00 & 26.50 & 31.06 & 26.01 & 0.98 \\
\hline \multirow[t]{3}{*}{ aqueous } & Min & 8.83 & 0.11 & 8.21 & 0.10 & 0.95 \\
\hline & Max & 59.65 & 126.65 & 62.13 & 130.67 & 1.03 \\
\hline & Avg & 19.38 & 6.44 & 19.32 & 6.45 & 1.00 \\
\hline \multirow[t]{3}{*}{ iris } & Min & 9.68 & 0.12 & 8.84 & 0.11 & 0.92 \\
\hline & Max & 39.50 & 34.23 & 39.85 & 34.45 & 1.01 \\
\hline & Avg & 19.34 & 4.63 & 18.86 & 4.53 & 0.98 \\
\hline \multirow[t]{3}{*}{ cornea } & Min & 9.51 & 0.12 & 7.56 & 0.11 & 0.87 \\
\hline & Max & 24.11 & 10.14 & 21.59 & 9.97 & 0.98 \\
\hline & Avg & 14.15 & 1.29 & 12.01 & 1.22 & 0.95 \\
\hline
\end{tabular}




\section{Chapter 4}

\section{Discussion}

\subsection{Verification of egs_brachy and eye plaque models}

The verification of egs_brachy and plaque models against other Monte Carlo calculations in Chapter 3 shows overall good agreement. While discrepancies exist in the doses calculated by the various Monte Carlo codes, they can be explained through small differences in how the codes operate, how the geometries are defined, and what data they use to make calculations. The source of cross sections and material composition data, the difference in volume correction algorithms, differences in geometry of models, and the assumptions made in the dose calculation methods all impact the final dose outcome for a given MC code and therefore result in a small discrepancy in calculated doses between codes.

\subsubsection{Photon plaque verification}

Comparisons between egs_brachy and BrachyDose show agreement that is generally within $1 \%$ across all plaque models. Figure 3.5 shows a minor systematic difference between egs_brachy and BrachyDose of about $0.6 \%$ for ${ }^{125} I$ seeds and $0.5 \%$ for

${ }^{103} P d$ seeds. This systematic uncertainty may arise from the differences in the scoring 
routines or modelling of geometries between the two codes. For other possible sources of the systematic discrepancy see 'Other uncertainties' in Section 2.4.1.

When looking at the $D_{w, w}$ results reported as absolute dose there are a few points of interest with large discrepancies $(\sim 20 \%)$ between egs_brachy and MCNP5. These points are shown in Table 3.2 and are located at the lacrimal gland for plaque positions \#1, \#2, and \#3. Looking at those same points for $D_{T G 43}$ in Table 3.1 it is shown that the large discrepancies seen in $D_{w, w}$ are no longer present. The implication is therefore that the introduction of the plaque model creates these discrepancies. This follows logically, as the lacrimal gland is located in the far penumbra region of the plaque for those positions (the penumbra is the dose to areas where radiation would either have to pass through the shielding or scatter into it to reach). It can be concluded that these discrepancies are likely caused by differences in the plaque geometry between egs_brachy and MCNP5. In Melhus and Rivard's 2008 and 2011 papers they confirm overall good agreement between MCNP5 and BrachyDose. egs_brachy shows very strong agreement with BrachyDose and similar agreement with MCNP5.

It is expected that $D_{T G 43}$ results would compare more favourably than $D_{w, w}$ as there is less room for discrepancy (if there is no plaque there can be no discrepancies in plaque geometry or composition), and this is the case when comparing egs_brachy and BrachyDose results. However; the results show similar or worse discrepancies between egs_brachy and MCNP5 for $D_{T G 43}$. A possible explanation for this is in the level of accuracy in emulating TG43. The exact configuration of the 'homogeneous' simulations for the $2008^{24}$ and $2011^{42}$ MCNP5 works is left somewhat ambiguous. In a personal communication with Melhus it was confirmed for the 'homogeneous' simulations in the 2008 paper, while the seeds were modelled in water without a plaque, interseeds effects were also modelled. egs_brachy has a superposition mode 
that simulates each seed individually and then combines the results to obtain a total dose, allowing for simulations to be done without interseed effects. The addition of interseed effects would affect the final doses calculated using MCNP5 and could result in larger discrepancies when comparing when comparing TG43 doses $\left(D_{T G 43}\right)$ than expected.

\subsubsection{Beta plaque verification}

There is solid agreement when comparing to beta plaque doses calculated by HermidaLopez using PENELOPE. The largest discrepancies are observed at locations that are either at depths near or past the $R_{C S D A}$ of $7 \mathrm{~mm}$ for the electron mean energy, 1.41 MeV, and/or within the penumbral region of the plaque. There are also a few outlier points where the PENELOPE calculations have a much higher dose than those calculated in egs_brachy. These outliers are observable in subfigure (b) of Figure 3.10 (the CCA plaque) and subfigure (a) of Figure 3.11 (the CCD plaque). These points are located along the lateral axis at a depth of $0.1 \mathrm{~cm}$. The dose is near to the plaque and located in voxels where the plaque intersects the scoring region.

These differences could be attributed to a differences in scoring methods, volume corrections or the modelling of geometries between PENELOPE and egs_brachy. Other than the outlier points most disagreements are less than $3 \%$ across all profiles for all plaques. The statistical uncertainties reach 2\%-2.5\% with egs_brachy and 1-2\% with PENELOPE at the far points which have $3 \%$ disagreement (a combined uncertainty of $3.2 \%$ ), meaning most disagreement can be explained through statistical uncertainties. The larger differences observed at points toward the eye centre are past the range of the mean electron energy, so little energy is deposited, resulting in low doses. With such low doses a small difference in dose value can result in a large 
difference in dose ratio. For example, at the same points most of the photon plaques have central axis doses of greater than $30 \mathrm{~Gy}$, while the beta plaque doses can be on the scale of a few (1-4) Gy.

There is a stronger agreement when comparing egs_brachy with the GEANT4 doses calculated by Sommer et al. ${ }^{49}$ in Figure 3.9. Up to the tumor apex there is only a $0.7 \%$ disagreement and, while the disagreement does reach $4.4 \%$ by $1 \mathrm{~cm}$, the actual dose difference at that point is only 0.13 Gy per decay. A stronger agreement between egs_brachy and GEANT4 doses is not unexpected as both use a model of the nuclide decay in their calculation ${ }^{49}$, while the PENELOPE doses were calculated using an ICRU spectrum ${ }^{47}$. When comparing with the BEBIG reference dataset disagreements of up to $7.5 \%$ with egs_brachy are observed. The GEANT4 results have similar discrepancies with the BEBIG reference. In either case the reported combined uncertainty for the BEBIG measurements is $20 \%$, accounting for far greater than $7.5 \%$ disagreements.

When looking at the CIA plaque which contains a cut-out notch, two different versions of the plaque were developed for this work. The 'CIA-HL' plaque is based on the work of Hermida-Lopez, and the 'CIA' plaque is developed in this work. The dose differences between the dose distributions calculated using the two different versions are not large, but are observable. Figure 3.12 shows that the central axis depth-dose curve and the $\mathrm{x}$-axis profiles for the two versions are within a $0.01 \%$ disagreement for most points. At a few points they have $2-3 \%$ disagreement or even greater disagreements of up to $20 \%$.

The larger disagreements are shown more clearly in the isodose contours of Figure 3.13 where a quite large discrepancy between CIA-HL and CIA can be observed near the plaque in the area of the cutout notch. This discrepancy shows higher doses 
near to the cutout for CIA over CIA-HL. The rounded region allows for the active source to cover a larger area of the plaque, and thus increases dose in that region. The disagreement between CIA and CIA-HL suggests that the more accurate CIA version should be used over the more simplistic CIA-HL version.

As seen in the depth-dose and cutout axis curves in Figure 3.12, comparing to PENELOPE shows the two egs_brachy CIA plaque versions result in doses that are in solid agreement. The rounded CIA model disagrees with PENELOPE at the same points and for similar percentages that it disagrees with the CIA-HL. This shows that the CIA-HL geometry created to mirror Hermida-Lopez is functioning properly as a clone, and confirms that rounding the edges of the cutout makes a difference in the dose calculations.

For BEBIG beta plaques accurate information of their physical geometry is difficult to find, especially with plaques that contain a cutout. This uncertainty in physical geometry could influence the difference seen in beta plaque dose distributions between various Monte Carlo codes.

\subsection{Comparison of $D_{T G 43}, D_{w, w}$, and $D_{m, m}$}

As introduced in Section 1.4, the probability of photon interaction at low energies is highly dependent on $Z_{\text {eff }}$ for the medium being traversed. This gives the expectation of large discrepancies between $D_{T G 43}$ and $D_{w, w}$. Reports such as TG12936 and TG186 ${ }^{87}$ have strongly recommended that clinics with the means include calculation of $D_{w, w}$ in their dose evaluation process. Other studies such as Melhus and Rivard's 2008 work $^{24}$ and Rivard et al's 2011 work $^{42}$ with MCNP5 and BrachyDose have shown results confirming the existence of large discrepancies between the two calculation types. This conclusion is supported by comparing the depth-dose curves 
of Figures 3.1 and 3.2 to those in Figures 3.3 and 3.4, as well as by looking at the ratios in Figure 3.7 and Table 3.3.

Figure 3.6 shows isodose contours of $D_{T G 43}$ alongside $D_{w, w}$ for different photon plaque models. The isodose contours show that $D_{T G 43}$ is consistently higher than $D_{w, w}$ for standard COMS $16 \mathrm{~mm}$ plaques as well as all the other plaque models except the "COMS - thin acrylic", which has a higher $D_{w, w}$ than $D_{T G 43}$ close to the plaque. That $D_{T G 43}$ is greater than $D_{w, w}$ shows the possibility of prescribing an underdose to a patient. For example, the results show a difference of $11 \%$ at the tumor apex between $D_{w, w}$ and $D_{T G 43}$; if $D_{T G 43}=85$ Gy is prescribed, then $D_{w, w}$ would only be 75.65 Gy. More extreme differences in $D_{T G 43}$ and $D_{w, w}$ can be observed in Table 3.3. The minimum dose to the optic nerve shows a $84 \%$ difference and the average dose to the optic nerve shows a $55 \%$ relative difference. These large discrepancies in dose calculations show a problematic lack of accuracy in clinical dose evaluations.

The isodose contours in Figure 3.6 and the ratios in subfigure (b) of Figure 3.7 show that differences between $D_{T G 43}$ and $D_{w, w}$ exist for each of the photon plaque models used in this thesis. It is interesting to note that $D_{w, w}$ calculated using the standard COMS plaque has a greater disagreement along the plaque central axis with $D_{T G 43}$ than all of the other photon plaque models. Understanding the geometry of the different plaques allows for an understanding of the differences in disagreement. The "COMS - thin acrylic" plaque would result in less photon attenuation than the standard COMS plaque as the seeds are just affixed to an acrylic layer on the backing, so the majority of photons leaving the plaque would not have to go through the medium of the insert. Because the "short lip - acrylic" plaque has a shorter lip there is less medium between the seeds and the eye and, therefore; less photon attenuation. The "no lip - silastic" plaque has the same height as the "short lip acrylic" plaque, so the seeds are closer to the eye than in the standard COMS plaque. 
The lipless plaque also has no high $Z_{\text {eff }}$ lip to attenuate the photons. The "stainless steel - acrylic" plaque's backing is stainless steel rather than a gold alloy, resulting in a much lower $Z_{\text {eff }}$ for the shielding of the plaque and less photon attentuation compared to the standard COMS plaque.

Lesperence et al. ${ }^{53}$ show that the addition of realistic tissue compositions and structures when calculating dose in the eye with photon plaques makes a significant difference. The ratios in Table 3.3 and Figure 3.7 and the isodose contours in Figure 3.6 show that similar differences observed in BrachyDose are achieved in egs_brachy as well. For example, Lesperance et al. report differences in average dose to the lens of $29 \%$, average dose to the vitreous of $14 \%$, and maximum dose to the optic nerve of $27 \%$ when comparing $D_{m, m}$ and $D_{T G 43}$. Table 3.3 shows that with egs_brachy dose differences in the same structures come to $29 \%, 14 \%$ and $28 \%$ respectively.

Table 3.3 shows a larger or similar difference in dose between $D_{m, m}$ and $D_{T G 43}$ to the ocular structures when compared to $D_{w, w}$ and $D_{T G 43}$. One of the most obvious differences between Figure 3.7(a) $\left(D_{w, w}\right.$ and $D_{T G 43}$ central axis ratios) and Figure 3.7(b) ( $D_{m, m}$ and $D_{T G 43}$ central axis ratios) is the behaviour within the tumor region. Within the tumor region $D_{m, m}$ is much higher than in $D_{w, w}$ and then drops off immediately after the tumor apex. Interestingly the tumor is the one structure where $D_{m, m}$ is much closer to $D_{T G 43}$ when using the standard COMS $16 \mathrm{~mm}$ plaque.

$D_{m, m}$ has a $95 \%$ agreement with $D_{T G 43}$ for the minimum, maximum and average dose to the tumor as shown in Table 3.3. Figure 1.2 shows at the average photon energy of $28 \mathrm{keV}$ for ${ }^{125} \mathrm{I}$, the mass-energy absorption coefficient is $\sim 10 \%$ higher for tumor than water. With a greater mass-energy absorption coefficient more photon attenuation occurs in the tumor medium. More photon attenuation leads to more energy being deposited in the tumor medium than would be in water. $D_{m, m}$ includes 
attenuation from the plaque model and interseed effects, while $D_{T G 43}$ does not, and so is still less than $D_{T G 43}$ in the tumor region.

Other eye structures can be analyzed in a similar manner. At $28 \mathrm{keV}$ the cornea, lens, and sclera have mass-energy absorption coefficients that are respectively, $8 \%$, $9 \%$, and $12 \%$ less than water. For each of these three structures $D_{m, m}$ is less than $D_{w, w}$. The lower $D_{m, m}$ concurs with the idea that a lower mass-energy absorption coefficent results in less photon attenuation and consequently, less energy deposition. The optic nerve has a mass-energy coefficient almost equivalent to water and so therefore $D_{w, w} \sim D_{m, m}$, which concurs with the idea that they should have equivalent photon attenuation. The iris, aqueous, and vitreous have mass-energy absorption coefficients that are approximately $2 \%, 5 \%, 6 \%$ more than water respectively, which suggests that $D_{m, m}$ should be higher than $D_{w, w}$ for these three eye structures. The max doses in each case are high for $D_{m, m}$, but only for vitreous is the average dose of $D_{m, m}$ greater than $D_{w, w}$. Photons must cross other media before reaching the iris or aqueous, so it is possible the attenuation in those upstream media result in less photons reaching the iris and aqueous, resulting in less energy deposition than expected.

Figure 3.15 and Table 3.5 allow for comparison of $D_{w, w}$ and $D_{m, m}$ for betaemitting plaques. Subfigure (c) of 3.15 shows isodose contours for $D_{w, w}$ and $D_{m, m}$ using a CCB beta plaque. The figure shows that $D_{w, w}$ is consistently slightly higher than $D_{m, m}$ for a beta plaque. Table 3.5 shows that when comparing $D_{w, w}$ and $D_{m, m}$ for a beta plaque, the difference in average dose for each eye structure is between $0 \%$ - $7 \%$. Looking at the stopping power ratios in Figure 1.3 explains these differences. The ratios of stopping powers in medium to water show that each of the media of the eye have slightly lower stopping powers than water (up to $2 \%$ lower with the tumor). Stopping power characterizes energy loss of a incident charged particle. A 
lower stopping power allows less energy to be deposited by a charged particle as it traverses the medium. Less energy deposition in the medium results in less less dose, which contributes to $D_{m, m}$ being consistently lower than $D_{w, w}$.

Clinically, dose evaluations are expected to be within a few percent of the actual dose that will be delivered. This is particularly relevant for organs at risk (OARs), where doses should be under a certain tolerance value to minimize damage to healthy tissues. Table 3.3 shows large discrepancies between $D_{T G 43}$ and both $D_{w, w}$ and $D_{m, m}$ that are far greater than a few percent. An example is the optic nerve, where $D_{T G 43}$ is different from $D_{m, m}$ by $55 \%$. For other OARs, the differences are closer to $20 \%$, and thus are clinically significant. $D_{T G 43}$ emulates clinical dose calculations, so the differences discussed show major clinical relevance.

\subsection{Comparison of photon and beta plaque dose distribu- tions}

As electrons are charged particles, they have a short range when traveling through most media. They undergo a large number of interactions and lose energy quickly. This is shown generally in Figure 3.14. Comparing beta plaques to photon plaques, the beta plaques have a higher dose within the tumor region that quickly drops off to nearly zero dose along the plaque central axis. isodose contours of a standard COMS $16 \mathrm{~mm}$ plaque and a CCB beta plaque are given in Figure 3.15. The isodose contours show how different the shape of the dose distribution is between photon and beta plaques. They also confirm how quickly dose calculated using a beta plaque drops off, not just along the central axis, but across the distribution.

Unfortunately, the short range of beta electrons in tissue leads to a "weakness" 
of the beta plaque. If a tumor is too 'tall' then the beta electrons may not be able to deposit an acceptable amount of energy throughout the entire tumor. Table 3.4 shows that this behaviour of electrons results in much lower doses to organs at risk than photon plaques for most plaque positions. It also shows, however, that with certain plaque placements the beta plaque will deliver much higher doses when the beta plaque is placed near the organ at risk. Table 3.5 shows minimum, maximum, and average doses to the tumor and the surrounding organs at risk. The table shows that the beta plaque offers lower minimum dose to the lens, sclera, optic nerve, and vitreous fluid; lower average doses except to the sclera and lower maximum doses to the lens and optic nerve for both $D_{w, w}$ and $D_{m, m}$. Within the tumor the beta plaque has higher maximum, minimum, and average doses than the photon plaque. The difference is larger for photon plaques, a logical conclusion to make based on Figures 1.2 and 1.3. These figures show a larger difference in mass-energy absorption coefficients between various eye tissues and water (with a largest difference of $~ 10 \%$ between tumor and water) than in stopping powers between the same tissues and water (the largest difference being $\sim 2 \%$ between tumor and water). 


\section{Chapter 5}

\section{Conclusions and outlook}

Current clinical photon eye plaque brachytherapy dose calculations are done using the TG43 formalism. The TG43 formalism for dose calculation involves several assumptions. All media are considered to be water rather than the high $Z$ materials of a plaque or the varying tissues of the human body. Additionally, the interseed effects, which account for a notable amount of photon attenuation, are ignored.

For beta plaques the situation is more ambiguous. No accepted standard for dose calculations and treatment planning has been put in place. Dose calculations for beta-emitting plaques can vary in accuracy and methodology. Questions as to the accuracy of doses calculated using the TG43 formalism have led to various studies on the effects of taking a model-based approach instead (modelling both the plaque and tissue compositions during dose calculation) and have concluded that there is a significant difference between TG43 calculations and model-based calculations. An investigation into various model-based dose calculation algorithms has ensued

and one of the most prominent candidates is the use of Monte Carlo-based dose calculations.

Through comparisons of $D_{T G 43}, D_{w, w}$, and $D_{m, m}$ this work has demonstrated the need for model-based calculations (purpose (i), section 1.6). This importance of more advanced model-based eye plaque brachytherapy dose calculations has been demon- 
strated many times, but implementation within the clinic will require access to well documented and thorough algorithms, plaque geometries, and material composition data. The plaque models presented in this work are developed within an open source geometry package (egs ++$)$ that works in conjunction with an open source Monte Carlo code (egs_brachy) and will be distributed with future releases of egs_brachy (purpose (ii), section 1.6). Through comparisons to other doses calculated by other Monte Carlo codes this work has verified egs_brachy and the plaque models against published results (purpose (iii), section 1.6). The availability of these plaque models will allow for future studies to investigate further aspects of eye plaque dose distributions and encourage the adoption of advanced model-based calculations by treatment planning systems and the clinics that use them. The variety of plaque models will also give a baseline for creation of more plaque models to be implemented within egs_brachy. Future studies will be able to investigate the difference between the various plaque types, their effects among the various ocular structures, and comparisons to other treatment modalities. Using the eye phantom, doses to volume such as those presented in Table 3.5 could be used to more accurately investigate the doses in ocular structures at chosen prescription doses. Such investigations could provide exploration of changes to prescription dose, including possible dose de-escalation. An example de-escalation study found decreased toxicity with equivalent tumor control using TG43 dose calculations but only a 69 Gy prescription dose ${ }^{91}$.

Model-based Monte Carlo calculations could also enable investigation into radiobiology for eye plaque brachytherapy. In 2012 Gagne et al. ${ }^{92}$ developed a method for evaluating model-based Monte Carlo dose distributions and the resultant biologicallyeffective dose distributions for photon plaques. While there is currently no analogous study for beta plaques, the geometries in this work could be used to accomplish one. Gagne et al. ${ }^{93}$ also investigated the radiobiological effects of implant duration and 
the choice of radionuclide for photon plaques. The study found that ${ }^{103} \mathrm{Pd}$ offered a radiobiological benefit when compared to ${ }^{125} \mathrm{I}$ and ${ }^{131} \mathrm{Cs}$; however, the magnitude of uncertainties eclipsed the significance of the differences. Similar studies could be done with egs_brachy and the plaques in this work to confirm or deny their results or to investigate the same effects from beta-emitting plaques.

This thesis work utilizes the egs_brachy Monte Carlo code and the egs ++ geometry package to create and verify various plaque models (both photon and beta-emitting) and investigate the use of a realistic eye phantom. The model-based approach also enables a comparison between a photon-emitting plaque and a betaemitting plaque to be performed with the goal of showcasing the versatility of research that the plaque models enable (purpose (iv), section 1.6). The created plaques will be distributed with future releases of egs_brachy as well as through a website database maintained by the CLRP. It is hoped they will be utilized in future studies, as a baseline for future plaque models, and to encourage the implementation of model-based dose calculations by treatment planning system developers and clinics. 


\section{Appendix A}

\section{Photon plaque seed positions}

The photon plaque designs in this thesis each have a different number of seeds and positions for those seeds. The seed positions for each of these plaque designs are presented in Table A.1. Seed positions are given in terms of $x_{c}, y_{c}$, and $z_{c}$, which are the $x, y$, and $z$ coordinates of the seed centres with respect to the eye plaque coordinate system. The angle of the seed $\theta$ which is measured from the x-axis is also included. 
Table A.1: Seed centre and seed angle coordinates for all photon plaques developed for this work. Seed angles are in relation to the $\mathrm{x}-\mathrm{y}$ plane where $0^{\circ}$ is a seed lying along the x-axis.

\begin{tabular}{|c|c|c|c|c|c|}
\hline Plaque size & $\begin{array}{c}\text { seed } \\
\text { number }\end{array}$ & $x_{c}(\mathrm{~cm})$ & $y_{c}(\mathrm{~cm})$ & $z_{c}(\mathrm{~cm})$ & $\theta(\mathrm{rad})$ \\
\hline \multirow{5}{*}{ COMS $10 \mathrm{~mm}$} & 1 & -0.230 & -0.230 & -0.201 & 2.36 \\
\hline & 2 & 0.230 & -0.230 & -0.201 & 0.78 \\
\hline & 3 & 0.230 & 0.230 & -0.201 & 5.50 \\
\hline & 4 & -0.230 & 0.230 & -0.201 & 3.93 \\
\hline & 5 & 0.000 & 0.000 & -0.240 & 4.71 \\
\hline \multirow[t]{8}{*}{ COMS $12 \mathrm{~mm}$} & 1 & -0.372 & -0.27 & -0.16 & 2.51 \\
\hline & 2 & 0.142 & -0.437 & -0.16 & 1.26 \\
\hline & 3 & 0.46 & 0 & -0.16 & 6.28 \\
\hline & 4 & 0.142 & 0.437 & -0.16 & 5.03 \\
\hline & 5 & -0.372 & 0.27 & -0.16 & 3.77 \\
\hline & 6 & 0 & -0.27 & -0.213 & 1.57 \\
\hline & 7 & 0 & 0.27 & -0.213 & 4.71 \\
\hline & 8 & 0 & 0 & -0.24 & 4.71 \\
\hline \multirow[t]{13}{*}{ COMS $14 \mathrm{~mm}$} & 1 & -0.59 & 0 & -0.106 & 3.14 \\
\hline & 2 & -0.295 & -0.511 & -0.106 & 2.09 \\
\hline & 3 & 0.295 & -0.511 & -0.106 & 1.05 \\
\hline & 4 & 0.59 & 0 & -0.106 & 0 \\
\hline & 5 & 0.295 & 0.511 & -0.106 & 5.24 \\
\hline & 6 & -0.295 & 0.511 & -0.106 & 4.19 \\
\hline & 7 & -0.41 & 0 & -0.177 & 3.14 \\
\hline & 8 & 0 & -0.41 & -0.177 & 1.57 \\
\hline & 9 & 0.41 & 0 & -0.177 & 0 \\
\hline & 10 & 0 & 0.41 & -0.177 & 4.71 \\
\hline & 11 & 0 & -0.21 & -0.224 & 1.57 \\
\hline & 12 & 0 & 0.21 & -0.224 & 4.71 \\
\hline & 13 & 0 & 0 & -0.24 & 4.71 \\
\hline \multirow{13}{*}{$\begin{array}{l}\text { COMS } 16 \mathrm{~mm} \\
\text { and } \\
\text { "COMS - thin } \\
\text { acrylic" }\end{array}$} & 1 & -0.568 & -0.273 & -0.087 & 2.69 \\
\hline & 2 & -0.14 & -0.614 & -0.087 & 1.8 \\
\hline & 3 & 0.393 & -0.493 & -0.087 & 0.89 \\
\hline & 4 & 0.63 & 0 & -0.087 & 0 \\
\hline & 5 & 0.393 & 0.493 & -0.087 & 5.39 \\
\hline & 6 & -0.14 & 0.614 & -0.087 & 4.48 \\
\hline & 7 & -0.568 & 0.273 & -0.087 & 3.59 \\
\hline & 8 & -0.45 & 0 & -0.164 & 3.14 \\
\hline & 9 & 0 & -0.45 & -0.164 & 1.57 \\
\hline & 10 & 0.45 & 0 & -0.164 & 6.28 \\
\hline & 11 & 0 & 0.45 & -0.164 & 4.71 \\
\hline & 12 & 0 & -0.18 & -0.228 & 1.57 \\
\hline & 13 & 0 & 0.18 & -0.228 & 4.71 \\
\hline
\end{tabular}




\begin{tabular}{|c|c|c|c|c|c|}
\hline Plaque size & $\begin{array}{c}\text { seed } \\
\text { number }\end{array}$ & $x_{c}(\mathrm{~cm})$ & $y_{c}(\mathrm{~cm})$ & $z_{c}(\mathrm{~cm})$ & $\theta(\mathrm{rad})$ \\
\hline \multirow{21}{*}{ COMS $18 \mathrm{~mm}$} & 1 & -0.77 & 0 & -0.003 & 3.14 \\
\hline & 2 & -0.544 & -0.544 & -0.003 & 2.36 \\
\hline & 3 & 0 & -0.77 & -0.003 & 1.57 \\
\hline & 4 & 0.544 & -0.544 & -0.003 & 0.78 \\
\hline & 5 & 0.77 & 0 & -0.003 & 6.28 \\
\hline & 6 & 0.544 & 0.544 & -0.003 & 5.5 \\
\hline & 7 & 0 & 0.77 & -0.003 & 4.71 \\
\hline & 8 & -0.544 & 0.544 & -0.003 & 3.92 \\
\hline & 9 & -0.62 & 0 & -0.092 & 3.14 \\
\hline & 10 & -0.31 & -0.537 & -0.092 & 2.09 \\
\hline & 11 & 0.31 & -0.537 & -0.092 & 1.04 \\
\hline & 12 & 0.62 & 0 & -0.092 & 6.28 \\
\hline & 13 & 0.31 & 0.537 & -0.092 & 5.23 \\
\hline & 14 & -0.31 & 0.537 & -0.092 & 4.19 \\
\hline & 15 & -0.318 & -0.318 & -0.164 & 2.36 \\
\hline & 16 & 0.318 & -0.318 & -0.164 & 0.78 \\
\hline & 17 & 0.318 & 0.318 & -0.164 & 5.5 \\
\hline & 18 & -0.318 & 0.318 & -0.164 & 3.92 \\
\hline & 19 & 0 & -0.2 & -0.225 & 1.57 \\
\hline & 20 & 0 & 0.2 & -0.225 & 4.71 \\
\hline & 21 & 0 & 0 & -0.24 & 4.71 \\
\hline \multirow[t]{24}{*}{ COMS $20 \mathrm{~mm}$} & 1 & -0.81 & -0.29 & 0.06 & 2.79 \\
\hline & 2 & -0.43 & -0.75 & 0.06 & 2.09 \\
\hline & 3 & 0.15 & -0.85 & 0.06 & 1.40 \\
\hline & 4 & 0.66 & -0.55 & 0.06 & 0.70 \\
\hline & 5 & 0.86 & 0.00 & 0.06 & 6.28 \\
\hline & 6 & 0.66 & 0.55 & 0.06 & 5.58 \\
\hline & 7 & 0.15 & 0.85 & 0.06 & 4.89 \\
\hline & 8 & -0.43 & 0.75 & 0.06 & 4.19 \\
\hline & 9 & -0.81 & 0.29 & 0.06 & 3.49 \\
\hline & 10 & -0.65 & -0.15 & -0.07 & 2.91 \\
\hline & 11 & -0.29 & -0.60 & -0.07 & 2.02 \\
\hline & 12 & 0.29 & -0.60 & -0.07 & 1.12 \\
\hline & 13 & 0.65 & -0.15 & -0.07 & 0.02 \\
\hline & 14 & 0.52 & 0.42 & -0.07 & 5.60 \\
\hline & 15 & 0.00 & 0.67 & -0.07 & 4.71 \\
\hline & 16 & -0.52 & 0.42 & -0.07 & 3.82 \\
\hline & 17 & -0.38 & -0.28 & -0.16 & 2.51 \\
\hline & 18 & 0.15 & -0.45 & -0.16 & 1.26 \\
\hline & 19 & 0.47 & 0.00 & -0.16 & 6.28 \\
\hline & 20 & 0.15 & 0.45 & -0.16 & 5.02 \\
\hline & 21 & -0.38 & 0.28 & -0.16 & 3.77 \\
\hline & 22 & 0.00 & -0.23 & -0.22 & 1.57 \\
\hline & 23 & 0.00 & 0.23 & -0.22 & 4.71 \\
\hline & 24 & 0.00 & 0.00 & -0.24 & 4.71 \\
\hline
\end{tabular}




\begin{tabular}{|c|c|c|c|c|c|}
\hline Plaque size & $\begin{array}{c}\text { seed } \\
\text { number }\end{array}$ & $x_{c}(\mathrm{~cm})$ & $y_{c}(\mathrm{~cm})$ & $z_{c}(\mathrm{~cm})$ & $\theta(\mathrm{rad})$ \\
\hline \multirow{21}{*}{ COMS $22 \mathrm{~mm}$} & 1 & -0.92 & 0.00 & 0.12 & 3.14 \\
\hline & 2 & -0.65 & -0.65 & 0.12 & 2.35 \\
\hline & 3 & 0.00 & -0.92 & 0.12 & 1.57 \\
\hline & 4 & 0.65 & -0.65 & 0.12 & 0.78 \\
\hline & 5 & 0.92 & 0.00 & 0.12 & 6.28 \\
\hline & 6 & 0.65 & 0.65 & 0.12 & 5.50 \\
\hline & 7 & 0.00 & 0.92 & 0.12 & 4.71 \\
\hline & 8 & -0.65 & 0.65 & 0.12 & 3.93 \\
\hline & 9 & -0.72 & -0.13 & -0.03 & 2.97 \\
\hline & 10 & -0.25 & -0.69 & -0.03 & 1.92 \\
\hline & 11 & 0.47 & -0.56 & -0.03 & 0.87 \\
\hline & 12 & 0.72 & 0.13 & -0.03 & 6.11 \\
\hline & 13 & 0.25 & 0.69 & -0.03 & 5.06 \\
\hline & 14 & -0.47 & 0.56 & -0.03 & 4.01 \\
\hline & 15 & -0.31 & -0.37 & -0.15 & 2.27 \\
\hline & 16 & 0.37 & -0.31 & -0.15 & 0.70 \\
\hline & 17 & 0.31 & 0.37 & -0.15 & 5.41 \\
\hline & 18 & -0.37 & 0.31 & -0.15 & 3.84 \\
\hline & 19 & 0.02 & -0.22 & -0.22 & 1.48 \\
\hline & 20 & -0.02 & 0.22 & -0.22 & 4.62 \\
\hline & 21 & 0.00 & 0.00 & -0.24 & 4.62 \\
\hline \multirow[t]{33}{*}{ COMS $24 \mathrm{~mm}$} & 1 & 0.00 & 0.00 & -0.24 & 1.57 \\
\hline & 2 & 0.00 & 0.19 & -0.23 & 1.57 \\
\hline & 3 & 0.00 & -0.19 & -0.23 & 4.71 \\
\hline & 4 & 0.44 & 0.09 & -0.16 & 2.95 \\
\hline & 5 & 0.06 & 0.45 & -0.16 & 1.7 \\
\hline & 6 & -0.41 & 0.19 & -0.16 & 0.44 \\
\hline & 7 & -0.31 & -0.33 & -0.16 & 5.53 \\
\hline & 8 & 0.22 & -0.39 & -0.16 & 4.21 \\
\hline & 9 & 0.47 & 0.45 & -0.08 & 2.37 \\
\hline & 10 & -0.06 & 0.64 & -0.08 & 1.48 \\
\hline & 11 & -0.54 & 0.35 & -0.08 & 0.58 \\
\hline & 12 & -0.61 & -0.20 & -0.08 & 5.96 \\
\hline & 13 & -0.22 & -0.61 & -0.08 & 5.07 \\
\hline & 14 & 0.34 & -0.55 & -0.08 & 4.17 \\
\hline & 15 & 0.64 & -0.08 & -0.08 & 3.27 \\
\hline & 16 & 0.83 & 0.07 & 0.04 & 3.05 \\
\hline & 17 & 0.59 & 0.59 & 0.04 & 2.36 \\
\hline & 18 & 0.07 & 0.83 & 0.04 & 1.66 \\
\hline & 19 & -0.48 & 0.68 & 0.04 & 0.96 \\
\hline & 20 & -0.80 & 0.21 & 0.04 & 0.26 \\
\hline & 21 & -0.75 & -0.35 & 0.04 & 5.85 \\
\hline & 22 & -0.35 & -0.75 & 0.04 & 5.15 \\
\hline & 23 & 0.21 & -0.80 & 0.04 & 4.45 \\
\hline & 24 & 0.68 & -0.48 & 0.04 & 3.75 \\
\hline & 25 & 0.91 & 0.45 & 0.21 & 2.69 \\
\hline & 26 & 0.41 & 0.93 & 0.21 & 1.99 \\
\hline & 27 & -0.28 & 0.98 & 0.21 & 1.29 \\
\hline & 28 & -0.84 & 0.57 & 0.21 & 0.59 \\
\hline & 29 & -1.01 & -0.11 & 0.21 & 3.04 \\
\hline & 30 & -0.71 & -0.73 & 0.21 & 5.42 \\
\hline & 31 & -0.07 & -1.01 & 0.21 & 4.78 \\
\hline & 32 & 0.60 & -0.82 & 0.21 & 4.08 \\
\hline & 33 & 0.98 & -0.25 & 0.21 & 3.38 \\
\hline
\end{tabular}




\begin{tabular}{|c|c|c|c|c|c|}
\hline Plaque size & $\begin{array}{c}\text { seed } \\
\text { number }\end{array}$ & $x_{c}(\mathrm{~cm})$ & $y_{c}(\mathrm{~cm})$ & $z_{c}(\mathrm{~cm})$ & $\begin{array}{c}\text { seed } \\
\text { angle } \\
(\mathrm{rad})\end{array}$ \\
\hline "Short lip - & 1 & -0.568 & -0.273 & -0.012 & 2.688 \\
acrylic" & 2 & -0.140 & -0.614 & -0.012 & 1.798 \\
and & 3 & 0.393 & -0.493 & -0.012 & 0.890 \\
"No lip - & 4 & 0.630 & 0.000 & -0.012 & 0.000 \\
silastic" & 5 & 0.393 & 0.493 & -0.012 & 5.393 \\
& 6 & -0.140 & 0.614 & -0.012 & 4.486 \\
& 7 & -0.568 & 0.273 & -0.012 & 3.595 \\
& 8 & -0.450 & 0.000 & -0.089 & 3.142 \\
& 9 & 0.000 & -0.450 & -0.089 & 1.571 \\
& 10 & 0.450 & 0.000 & -0.089 & 6.283 \\
& 11 & 0.000 & 0.450 & -0.089 & 4.712 \\
& 12 & 0.000 & -0.180 & -0.153 & 1.571 \\
& 13 & 0.000 & 0.180 & -0.153 & 4.712 \\
\hline "Stainless steel - & 1 & -0.568 & -0.273 & -0.058 & 2.688 \\
& 2 & -0.140 & -0.614 & -0.058 & 1.798 \\
& 3 & 0.393 & -0.493 & -0.058 & 0.890 \\
& 4 & 0.630 & 0.000 & -0.058 & 0.000 \\
& 5 & 0.393 & 0.493 & -0.058 & 5.393 \\
& 6 & -0.140 & 0.614 & -0.058 & 4.486 \\
& 7 & -0.568 & 0.273 & -0.137 & 3.595 \\
& 8 & -0.450 & 0.000 & -0.137 & 3.142 \\
& 9 & 0.000 & -0.450 & -0.137 & 1.571 \\
& 10 & 0.450 & 0.000 & -0.137 & 6.283 \\
& 11 & 0.000 & 0.450 & -0.137 & 4.712 \\
& 12 & 0.000 & -0.180 & -0.203 & 1.571 \\
& 13 & 0.000 & 0.180 & -0.203 & 4.712 \\
\hline \hline
\end{tabular}




\section{References}

[1] C. L. Shields and J. A. Shields, Ocular melanoma: relatively rare but requiring respect, Clin. Dematol. 27, 122 - 133 (2009). (p 1)

[2] B. Tarlan and H. Kiratli, Uveal Melanoma: Current Trends in Diagnosis and Management, Turk. J. Ophthalmol 46, 123 - 137 (2016). (p 1)

[3] O. Pastyr, G. H. Hartmann, W. Schlegel, S. Schabbert, H. Treuer, W. J. Lorenz, and V. Sturm, Stereotactically guided convergent beam irradiation with a linear accelerator: Localization-technique, Acta Neurochir. 99, 61 - 64 (1989). (p 1)

[4] K. Muller, P. J. Nowak, C. de Pan, J. P. Marijnissen, D. A. Paridaens, P. Levendag, and G. P. Luyten, Effectiveness of fractionated stereotactic radiotherapy for uveal melanoma, Med. Phys. 30, 116-122 (2005). (p 1)

[5] E. S. Gragoudas, M. Goitein, A. M. Koehler, L. Verhey, J. Tepper, H. D. Suit, R. Brockhurst, and I. J. Constable, Proton irradiation of small choroidal malignant melanomas, Am. J. Ophthalmol 83, 665 - 673 (1977). (p 1)

[6] E. S. Gragoudas, M. Goitein, L. Verhey, J. Munzenreider, M. Urie, H. D. Suit, and A. M. Koehler, Proton beam irradiation of uveal melanomas. Results of 5 and a half year study, Arch. Ophthalmol 100, 928 - 934 (1982). (p 1)

[7] M. W. Wilson and J. L. Hungerford, Comparison of episcleral plaque and proton beam radiation therapy for the treatment of choroidal melanom, Ophthalmology 106, 1579 - 1587 (1999). (p 1)

[8] B. Damato, A. Kacperek, M. Chopra, I. R. Campbell, and R. D. Errington, Proton beam radiotherapy of choroidal melanoma: the Liverpool-Clatterbridge experience, Int. J. Radiat. Oncol. Biol. Phys. 62, 1405 - 1411 (2005). (p 1)

[9] D. H. Char, S. M. Kroll, and J. Castro, Ten-year follow-up of helium ion therapy for uveal melanoma, Am. J. Ophthalmol 125, 81 - 89 (1998). (p 1)

[10] S. Logani, T. K. Helenowski, H. Thakrar, and B. Pothiawala, Gamma Knife radiosurgery in the treatment of ocular melanoma, Stereotact. Funct. Neurosurg 61S1, $38-44$ (1993). (p 1)

[11] P. E. Carvounis and B. Katz, Gamma knife radiosurgery in neuroophthalmology, Curr. Opin. Ophthalmol 14, 317 - 324 (2003). (p 1) 
[12] G. Modorati, E. Miserocchi, L. Galli, P. Picozzi, and P. Rama, Gamma knife radiosurgery for uveal melanoma: 12 years of experience, Br. J. Ophthalmol. 93, $40-44$ (2009). (p 1)

[13] E. B. Podgorsak, Radiation Physics for Medical Physicists (2nd Ed), SpringerVerlag, Berlin, Heidelberg, 2010. (p 2)

[14] E. J. Hall and A. J. Giaccia, Radiobiology for the Radiologist, 7th ed., Lippincott Williams \& Wilkins, Philadelphia, 2012. (p 2)

[15] D. Eriksson and T. Stigbrand, Radiation-induced cell death mechanisms, Tumor Biol. 31, $363-372$ (2010). (p 2)

[16] L. L. Anderson et al., Interstitial Brachytherapy: Physical, Biological, and Clinical Considerations, Raven Press, New York, 1990. (p 2)

[17] R. Moore, Choroidal sarcoma treated by the intraocular insertion of radon seeds, Br. J. Ophthalmol 14, 145-156 (1930). (p 3)

[18] H. B. Stallard, Radiotherapy for malignant melanoma of the choroid, Br. J. Ophthalmol 50, 147 - 155 (1966). (p 3)

[19] P. K. Lommatzsch, Results after beta-irradiation (Ru-106/Rh-106) of choroidal melanomas. Twenty years experience., A. J. Clin. Oncol. 10, 146 - 151 (1987). (p 3)

[20] B. Damato, I. Patel, I. R. Campbel, H. M. Maylesl, and R. D. Errington, Local tumor control after Ru-106 brachytherapy of choroidal melanoma, Int. J. Radiat. Oncol. Biol. Phys. 63, 385 - 391 (2005). (p 3)

[21] A. F. Brovkina, G. D. Zarubei, and V. V. Val'skii, Criteria for assessing the efficacy of brachytherapy of uveal melanomas, complications of therapy and their prevention., Vestnikoftalmologii 113, 14 - 16 (1997). (p 3)

[22] R. Sealy, P. L. le Roux, F. Rapley, E. Hering, D. Shackleton, and D. Sevel, The treatment of ophthalmic tumours with low-energy sources, Br. J. Radiol. 49, $551-554$ (1976). (p 3)

[23] S. Packer and M. Rotman, Radiotherapy of choroidal melanoma with iodine125, Ophthalmology 8, $582-590$ (1980). (p 3)

[24] C. S. Melhus and M. J. Rivard, COMS eye plaque brachytherapy dosimetry simulations for ${ }^{103} \mathrm{Pd},{ }^{125} \mathrm{I}$, and ${ }^{131} \mathrm{Cs}$, Med. Phys. 35, $3364-3371$ (2008). (pp $3,13,38,41,42,43,72$, and 75$)$

[25] K. L. Leonard, N. L. Gagne, J. E. Mignano, J. S. Duker, E. A. Bannon, and M. J. Rivard, A 17-year retrospective study of institutional results for eye plaque brachytherapy of uveal melanoma using I-125, Pd-103, and Cs-131 and historical perspective, Brachytherapy 10, 331 - 339 (2011). (p 3) 
[26] P. T. Finger, K. J. Chin, and G. Duvall, Palladium-103 for Choroidal Melanoma Study G: Palladium-103 ophthalmic plaque radiation therapy for choroidal melanoma: 400 treated patients, Ophthalmology 116, 790 - 796 (2009). (p 3)

[27] N. Murakami, S. Suzuki, Y. Ito, R. Yoshimura, K. Inaba, Y. Kuroda, M. Morota, H. Mayahara, M. Sakudo, A. Wakita, H. Okamoto, M. Sumi, Y. Kagami, K. Nakagawa, K. Ohtomo, and J. Itami, Ruthenium-106 plaque therapy (RPT) for retinoblastoma, Int. J. Radiat. Oncol. Biol. Phys. 84, 59 - 65 (2012). (p 3)

[28] Karaiskos, P. Papagiannis, L. Sakelliou, G. Anagnostopoulos, and D. Baltas, Monte Carlo dosimetry of the selectSeed ${ }^{125}$ I interstitial brachytherapy seed, Med. Phys. 28, 1753-1760 (2001). (p 3)

[29] COMS Group, COMS manual of procedures, Ch 12: Radiation therapy, COMS manual of procedures PB95-179693, Springfield, VA: National Technical Information Service, 1995. (p 3)

[30] COMS Group, The COMS randomized trial of iodine 125 brachytherapy for choroidal melanom. V. Twelve-year mortality rates and prognostic factors: COMS report no. 28, Arch. Ophthalmol. 124, 1684-1693 (2006). (p 3)

[31] A. J. Lin, Y. J. Rao, S. Acharya, J. Schwarz, P. K. Rao, and P. Grigsby, Patterns of care and outcomes of proton and eye plaque brachytherapy for uveal melanoma: Review of the National Cancer Database, Brachytherapy 16, 1225 - 1231 (2017). (p 4)

[32] J. Peer, Ruthenium-106 brachytherapy, Dev. Ophthalmol 49, 27 - 40 (2012). (p 4)

[33] R. Nath, L. L. Anderson, G. Luxton, K. A. Weaver, J. F. Williamson, and A. S. Meigooni, Dosimetry of interstitial brachytherapy sources: Recommendations of the AAPM Radiation Therapy Committee Task Group No. 43, Med. Phys. 22, $209-234$ (1995). (p 4)

[34] M. J. Rivard, B. M. Coursey, L. A. DeWerd, M. S. Huq, G. S. Ibbott, M. G. Mitch, R. Nath, and J. F. Williamson, Update of AAPM Task Group No. 43 Report: A revised AAPM protocol for brachytherapy dose calculations, Med. Phys. 31, 633 - 674 (2004). (pp 4, 31, and 41)

[35] S. Nag, J. M. Quivey, J. D. Earle, D. Followill, J. Fontanesi, and P. T. Finger, The American Brachytherapy Society recommendations for brachytherapy of uveal melanomas, Int. J. Radiat. Oncol. Biol. Phys. 56, $544-555$ (2003). (p 4)

[36] S.-T. Chiu-Tsao et al., Dosimetry of ${ }^{125} \mathrm{I}$ and ${ }^{103} \mathrm{Pd}$ COMS eye plaques for intraocular tumors: Report of Task Group 129 by the AAPM and ABS, Med. Phys. 39, 6161 - 6184 (2012). (pp 7, 20, and 75) 
[37] L. Beaulieu, A. C. Tedgren, J.-F. Carrier, S. D. Davis, F. Mourtada, M. J. Rivard, R. M. Thomson, F. Verhaegen, T. A. Wareing, and J. F. Williamson, Report of the Task Group 186 on model-based dose calculation methods in brachytherapy beyond the TG-43 formalism: Current status and recommendations for clinical implementation, Med. Phys. 39, 6208-6236 (2012). (p 7)

[38] H. E. Johns and J. R. Cunningham, The physics of radiology, 4th ed., Charles C. Thomas, Springfield, Illinois, 1983. (p 8)

[39] M. J. Berger, ESTAR, PSTAR and ASTAR: Computer Programs for Calculating Stopping-Power and Ranges for Electrons, Protons, and Helium Ions, NIST Report NISTIR-4999 (Washington DC), available on-line at http://physics.nist.gov/Star (1992). (p 12)

[40] G. Yegin, R. E. P. Taylor, and D. W. O. Rogers, BrachyDose: A New Fast Monte Carlo Code for Brachytherapy Calculations, Med. Phys. 33, 2074 - 2074 (abs) (2006). (p 13)

[41] R. M. Thomson, R. E. P. Taylor, and D. W. O. Rogers, Monte Carlo dosimetry for ${ }^{125} \mathrm{I}$ and ${ }^{103} \mathrm{Pd}$ eye plaque brachytherapy, Med. Phys. 35, 5530 - 5543 (2008). (p 13)

[42] M. J. Rivard et.al., Comparison of dose calculation methods for brachytherapy of intraocular tumors, Med. Phys. 38, 306 - 316 (2011). (pp 13, 41, 42, 43, 72, and 75)

[43] R. M. Thomson, R. E. P. Taylor, and D. W. O. Rogers, The CLRP Eye Plaque Database, http://www.physics.carleton.ca/clrp/eye_plaque . (pp 13, 41, and 42)

[44] F. B. Brown (Editor), MCNP-A general Monte Carlo N-particle transport code Version 5, Report LA-UR-03-1987, Los Alamos National Laboratory, Los Alamos, NM, 2003. (p 13)

[45] H. Zhang, D. Martin, S.-T. Chiu-Tsao, A. Meigooni, and B. R. Thomadsen, A comprehensive dosimetric comparison between ${ }^{131} \mathrm{I}$ and ${ }^{125} \mathrm{I}$ brachytherapy sources for COMS eye plaque implant, Brachytherapy 9, $362-372$ (2010). (p 13)

[46] J. Baro, J. Sempau, J. M. Fernandez-Varea, and F. Salvat, PENELOPE: an algorithm for Monte Carlo simulation of the penetration and energy loss of electrons and positrons in matter, Nucl. Inst. Meth. B 100, $31-46$ (1995). (p 13)

[47] M. Hermida-López, Calculation of dose distributions for $12{ }^{106} \mathrm{Ru} /{ }^{106} \mathrm{Rh}$ ophthalmic applicator models with the PENELOPE Monte Carlo code, Med. Phys. 40, 101705(13pp) (2013), 101705. (pp 13, 30, 41, 42, 58, 59, 64, 65, and 74) 
[48] S. Agostinelli et al., GEANT4 - a simulation toolkit, Nucl. Inst. Meth. A 506, $250-303$ (2003). (p 13)

[49] H. Sommer, M. Ebenau, B. Spaan, and M. Eichmann, Monte Carlo simulation of ruthenium eye plaques with GEANT4: influence of multiple scattering algorithms, the spectrum and the geometry on depth dose profles, Phys. Med. Biol. 62, 1848 - 1864 (2017). (pp 13, 38, 41, 42, 58, and 74)

[50] R. M. Thomson and D. W. O. Rogers, Monte Carlo dosimetry for ${ }^{125} \mathrm{I}$ and ${ }^{103} \mathrm{Pd}$ eye plaque brachytherapy with various seed models, Med. Phys. 37, 368 - 376 (2010). (p 13)

[51] M. A. Astrahan, A. Szechter, and P. T. Finger, Design and dosimetric considerations of a modified COMS plaque: The reusable "seed-guide" insert, Med. Phys. 32, 2706 - 2716 (2005). (pp 14 and 24)

[52] M. Lesperance, M. Martinov, and R. M. Thomson, Monte Carlo dosimetry for ${ }^{103} \mathrm{Pd},{ }^{125} \mathrm{I}$, and ${ }^{131} \mathrm{Cs}$ ocular brachytherapy with various plaque models using an eye phantom, Med. Phys. 41, 031706 (14pp) (2014). (pp 14, 23, 39, and 54)

[53] M. Lesperance, M. Inglis-Whalen, and R. M. Thomson, Model-based dose calculations for COMS eye plaque brachytherapy using an anatomically realistic eye phantom, Med. Phys. 41, 021717 (12pp) (2014). (pp 14, 32, 34, and 77)

[54] M. Chamberland, R. E. P. Taylor, D. W. O. Rogers, and R. M. Thomson, egs_brachy: a versatile and fast Monte Carlo code for brachytherapy, Phys. Med. Biol. 61, 8214 - 8231 (2016). (pp 14 and 26)

[55] J. A. Martín-Viera Cueto, V. Parra Osorio, C. Moreno Sáiz, F. Navarro Guirado, F. J. Casado Villalón, and P. Galán Montenegro, A universal dose-response curve for radiochromic films, Med. Phys. 42, 221 - 231 (2015). (p 20)

[56] S. E. M. Cutsinger, K. M. Furutani, R. M. Forsman, and S. M. Corner, Seed coordinates of a new COMS-like $24 \mathrm{~mm}$ plaque verified using the FARO Edge, J Appl Clin Med Phys 16, 293 - 301 (2015). (p 21)

[57] P. T. Finger, A. Berson, and A. Szechter, Palladium-103 plaque radiotherapy for choroidal melanoma, Ophthalmology 106, 606 - 613 (1999). (p 24)

[58] H. Zhang, F. Davidorf, and Y. Qi, Comparison of $16 \mathrm{~mm}$ OSU-Nah and COMS eye plaques, J of App Clin Med Phys 13, 166 - 178 (2012). (p 24)

[59] I. Puusaari, J. Heikkonen, and T. Kivela, Ocular complications after iodine brachytherapy for large uveal melanomas, Opthalmology 111, 1768 - 1777 (2004). (p 24)

[60] S. Nag, D. Wang, H. Wu, C. J. Bauer, R. B. Chambers, and F. H. Davidorf, Custom-made "Nag" eye plaques for ${ }^{125} \mathrm{I}$ brachytherapy, Int. J. Radiat. Oncol. Biol. Phys. 56, 1373 - 1380 (2003). (p 24) 
[61] D. Granero, J. Perez-Calatayud, F. Ballester, and E. Casal, Dosimetric study of the 15 mm ROPES eye plaques, Med Phys 31, 3330 - 3336 (2004). (p 25)

[62] C. Karolis, R. Frost, and F. Billson, A thin I-125 seed eye plaque to treat intraocular tumors using an acrylic insert to precisely position the sources, J. Radiat. Oncol. Biol. Phys. 18, 1209 - 1213 (1990). (p 25)

[63] J. F. Williamson, Comparison of measured and calculated dose rates in water near I-125 and Ir-192 seeds, Med. Phys. 18, 776 - 786 (1991). (p 26)

[64] J. Dolan, Z. Li, and J. F. Williamson, Monte Carlo and experimental dosimetry of an ${ }^{125}$ I brachytherapy seed, Med. Phys. 33, 4675 - 4684 (2006). (p 26)

[65] Z. Chen and R. Nath, Dose rate constant and energy spectrum of interstitial brachytherapy sources, Med. Phys. 28, 86 - 96 (2001). (p 26)

[66] J. I. Monroe and J. F. Williamson, Monte Carlo-aided dosimetry of the Theragenics TheraSeed Model $200{ }^{103} \mathrm{Pd}$ interstitial brachytherapy seed, Med. Phys. 29, $609-621$ (2002). (pp 26 and 27)

[67] R. E. P. Taylor, G. Yegin, and D. W. O. Rogers, Benchmarking BrachyDose: voxel-based EGSnrc Monte Carlo calculations of TG-43 dosimetry parameters, Med. Phys. 34, 445 - 457 (2007). (p 26)

[68] NCRP Report 58, A Handbook of Radioactivity Measurements Procedures, NCRP Publications, 7910 Woodmont Avenue, Bethesda, MD. 20814 USA (1985). (p 26)

[69] M. Rodriguez and D. W. O. Rogers, On determining dose rate constants spectroscopically, Med. Phys. 40, 011713 (10pp) (2013). (pp 26 and 27)

[70] Brookhaven National Laboratory, National Nuclear Data Center, NuDat 2.7, http://www.nndc.bnl.gov/nudat2 . (p 27)

[71] ICRP, Adult Reference Computational Phantoms, ICRP Report 110, International Commission on Radiological Protection, Washington D.C., 2009. (p 32)

[72] M. J. Hogan, J. A. Alvarado, and J. E. Weddell, Histology of the human eye: an atlas and textbook, W. B. Saunders Company, Philadelphia, 1971. (pp 32 and 33)

[73] F. A. Jakobiec, Ocular Anatomy, Embryology and Teratology, Philadelphia, 1982. (pp 32 and 33)

[74] H. Davson, The Eye, Academic Press, Inc., Orlando, FL, 3rd edition, 1984. (p 32)

[75] K. Meek, The cornea and Sclera, in Collagen: Structure and Mechanics, pages 359-396 (2008). (p 33) 
[76] H. Q. Woodard and D. R. White, The composition of body tissues, Brit. J. Radiol. 59, 1209 - 1219 (1986). (p 33)

[77] A. Presland, Applied ocular physiology and anatomy, Anaesth. Intensive Care 8, $379-382$ (2007). (p 33)

[78] N. K. Tumram, R. V. Bardale, and A. P. Dongre, Postmortem analysis of synovial fluid and vitreous humour for determination of death interval: A comparative study, Forensic Sci. Int. 204, 186-190 (2011). (p 33)

[79] E. R. Berman, Biochemistry of the Eye, (1991). (p 33)

[80] ICRU, Electron, Photon and Neutron Interaction Data for Body Tissues, ICRU Report 46, International Commission on Radiation Units and Measurements, Washington D.C., 1992. (p 33)

[81] R. L. Maughan, P. J. Chuba, A. T. Porter, E. Ben-Josef, and D. R. Lucas, The elemental composition of tumors: Kerma data for neutrons, Med. Phys. 24, 1241 - 1244 (1997). (p 33)

[82] J. F. Williamson, Monte Carlo evaluation of kerma at a point for photon transport problems, Med. Phys. 14, 567 - 576 (1987). (p 35)

[83] R. M. Thomson, et.al., User manual for egs_brachy: A versatile and fast EGSnrc application for brachytherapy, (2017). (pp 35, 36, and 37)

[84] B. R. B. Walters, I. Kawrakow, and D. W. O. Rogers, History by history statistical estimators in the BEAM code system, Med. Phys. 29, 2745 - 2752 (2002). (p 36)

[85] M. J. Berger, J. H. Hubbell, and S. M. S. et al., XCOM: Photon cross section database (version 1.5), Technical Report NBSIR87-3597, NIST, Gaithersburg, MD, http://physics.nist.gov/xcom, 2010. (p 37)

[86] M. Hermida-Lopez, Calculation of dose distributions for 12 Ru-106/Rh-106 opthalmic applicator models with the PENELOPE Monte Carlo code, Med. Phys. 40, 101705 (13pp) (2013). (p 38)

[87] L. Beaulieu et al., Report of the Task Group 186 on model-based dose calculation methods in brachytherapy beyond the TG-43 formalism: Current status and recommendations for clinical implementation, Med. Phys. 39, 6208 - 6236 (2012). (pp 39 and 75)

[88] Y. Ma et al., A generic TG-186 shielded applicator for commissioning modelbased dose calculation algorithms for hig-dose-rate ${ }^{192} \mathrm{Ir}$ brachytherapy, Med. Phys. 44, 5961 - 5976 (2017). (p 40)

[89] R. M. Thomson, R. E. P. Taylor, M. J. P. Chamberland, and D. W. O. Rogers, Reply to Comment on 'egs_brachy: a versatile and fast Monte Carlo code for brachytherapy', Phys. Med. Biol. 63, 038002(5pp) (2018). (p 40) 
[90] BEBIG, Ru-106 eye applicators, Technical report, Eckert \& Ziegler BEBIG GmbH, Berlin, Germany, 2013. (p 58)

[91] B. A. Perez, P. Mettu, L. Vajzovic, D. Rivera, A. Alkaissi, B. A. Steffey, J. Cai, S. Stinnett, J. J. Dutton, E. G. Buckley, E. Halperin, L. B. Marks, P. Mruthyunjaya, and D. G. Kirsch, Uveal melanoma treated with iodine-125 episcleral plaque: An analysis of dose on disease control and visual outcomes, Int J Radiat Oncol 89, 127 - 136 (2014). (p 82)

[92] N. L. Gagne, K. L. Leonard, K. E. Huber, J. E. Mignano, J. S. Duker, N. V. Laver, and M. J. Rivard, BEDVH - A method for evaluating biologically effective dose volume histograms: Application to eye plaque brachytherapy implants, Med Phys 39, 976 - 983 (2012). (p 82)

[93] N. L. Gagne, K. L. Leonard, and M. J. Rivardl, Radiobiology for eye plaque brachytherapy and evaluation of implant duration and radionuclide choice using an objective function, Med Phys 39, 3332 - 3342 (2012). (p 82) 